Florida International University

FIU Digital Commons

FIU Electronic Theses and Dissertations

University Graduate School

7-9-2008

\title{
Peak-to-Average Power Ratio Reduced Parallel Interference Cancellation Multicarrier-Code Division Multiple Access System with Anti- Interference Property
}

Jun Luo

Florida International University, jluo001@fiu.edu

DOI: $10.25148 /$ etd.FI08121908

Follow this and additional works at: https://digitalcommons.fiu.edu/etd

\section{Recommended Citation}

Luo, Jun, "Peak-to-Average Power Ratio Reduced Parallel Interference Cancellation Multicarrier-Code Division Multiple Access System with Anti-Interference Property" (2008). FIU Electronic Theses and Dissertations. 20.

https://digitalcommons.fiu.edu/etd/20 


\section{FLORIDA INTERNATIONAL UNIVERSITY}

Miami, Florida

PEAK-TO-AVERAGE POWER RATIO REDUCED PARALLEL INTERFERENCE CANCELLATION MULTICARRIER-CODE DIVISION MULTIPLE ACCESS SYSTEM WITH ANTI-INTERFERENCE PROPERTY

A dissertation submitted in partial fulfillment of the requirements for the degree of DOCTOR OF PHILOSOPHY

in

ELECTRICAL ENGINEERING

by

Jun Luo 
To: Interim Dean Amir Mirmiran

College of Engineering and Computing

This dissertation, written by Jun Luo, and entitled Peak-to-Average Power Ratio Reduced Parallel Interference Cancellation Multicarrier-Code Division Multiple Access System with Anti-interference Property, having been approved in respect to style and intellectual content, is referred to you for judgment.

We have read this dissertation and recommend that it be approved.

Kang K. Yen

$\begin{array}{r}\text { Hao Zhu } \\ \hline \text { Shih-Ming Lee }\end{array}$

Date of Defense: July 9, 2008

The dissertation of Jun Luo is approved.

Interim Dean Amir Mirmiran
College of Engineering and Computing

Florida International University, 2008 


\section{DEDICATION}

To My Family and

To My New Born Daughter Specially 


\section{ACKNOWLEDGMENTS}

I would like to express my gratitude to all those who gave the possibility to complete this thesis.

First of all, there are no words enough to express my gratitude to my advisor, Dr. Jean H. Andrian who not only inspired and guided me throughout my academic program but also served as a mentor in my life. Without his constant support and encouragement I could never have finished my Ph.D. program.

Besides my advisor, I would like to thank my dissertation committee: Dr. Hao Zhu, Dr. Shih-Ming Lee, and Dr. Kang K. Yen for all their help. Especially for Dr. Kang K. Yen, without his help and support toward me I would have never got through my Ph.D. program.

I want to say thanks to the current and former student members in my Lab: Omar Granados, Huijing Qiang, Mi Wang, Kefeng Tan, Juan Chen and Changan Han, who gave me a stimulating and fun environment in which to learn and grow for the three years. My appreciation also goes to Pat Brammer, Maria Benincasa, Ana Saenz, and Xiang Li, for their kind administrative support. The special gratitude goes to Dr. Te-shun Chou as well since we had a wonderful collaboration on the Intrusion Detection Project.

This thesis is dedicated to my parents. They always encourage me to move forward and take care of my life in China. I want to also say thanks to my wife Xiaomei Bian. She gave up her medical doctor career and came to US with me. Because of her I never feel lonely in US. I appreciate my mother-in-law too. Without her help during this period, I can't finish my thesis on time. 
On Mar. 5th 2008, a new life came to this world, my daughter Rebekah L. Luo. She gives a lot of fun to my life. She will be the most important part in my world. My daughter, this work is for you, specially.

Finally, may all people be blessed with disaster-free life, especially for the people in Sichuan, China. 


\section{ABSTRACT OF THE DISSERTATION \\ PEAK-TO-AVERAGE POWER RATIO REDUCED PARALLEL INTERFERENCE \\ CANCELLATION MULTICARRIER-CODE DIVISION MULTIPLE ACCESS \\ SYSTEM WITH ANTI-INTERFERENCE PROPERTY}

by

Jun Luo

Florida International University, 2008

Miami, Florida

Professor Jean H. Andrian, Major Professor

Orthogonal Frequency-Division Multiplexing (OFDM) has been proved to be a promising technology that enables the transmission of higher data rate. Multicarrier Code-Division Multiple Access (MC-CDMA) is a transmission technique which combines the advantages of both OFDM and Code-Division Multiplexing Access (CDMA), so as to allow high transmission rates over severe time-dispersive multi-path channels without the need of a complex receiver implementation. Also MC-CDMA exploits frequency diversity via the different subcarriers, and therefore allows the high code rates systems to achieve good Bit Error Rate (BER) performances. Furthermore, the spreading in the frequency domain makes the time synchronization requirement much lower than traditional direct sequence CDMA schemes.

There are still some problems when we use MC-CDMA. One is the high Peak-toAverage Power Ratio (PAPR) of the transmit signal. High PAPR leads to nonlinear distortion of the amplifier and results in inter-carrier self-interference plus out-of-band radiation. On the other hand, suppressing the Multiple Access Interference (MAI) is 
another crucial problem in the MC-CDMA system. Imperfect cross-correlation characteristics of the spreading codes and the multipath fading destroy the orthogonality among the users, and then cause MAI, which produces serious BER degradation in the system. Moreover, in uplink system the received signals at a base station are always asynchronous. This also destroys the orthogonality among the users, and hence, generates MAI which degrades the system performance. Besides those two problems, the interference should always be considered seriously for any communication system. In this dissertation, we design a novel MC-CDMA system, which has low PAPR and mitigated MAI. The new Semi-blind channel estimation and multi-user data detection based on Parallel Interference Cancellation (PIC) have been applied in the system. The Low Density Parity Codes (LDPC) has also been introduced into the system to improve the performance. Different interference models are analyzed in multi-carrier communication systems and then the effective interference suppression for MC-CDMA systems is employed in this dissertation. The experimental results indicate that our system not only significantly reduces the PAPR and MAI but also effectively suppresses the outside interference with low complexity. Finally, we present a practical cognitive application of the proposed system over the software defined radio platform. 


\section{TABLE OF CONTENTS}

CHAPTER

PAGE

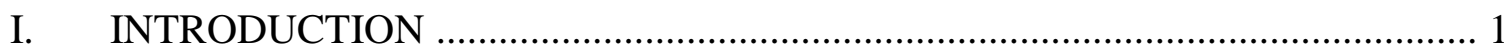

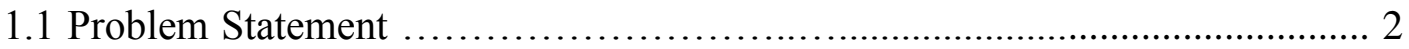

1.2 Research Hypotheses ......................................................................... 4

1.3 Proposed Approach .......................................................................

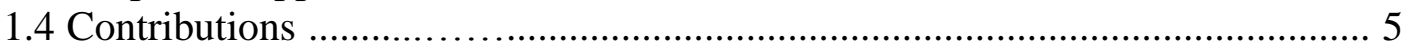

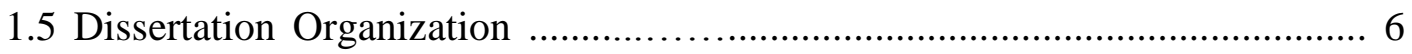

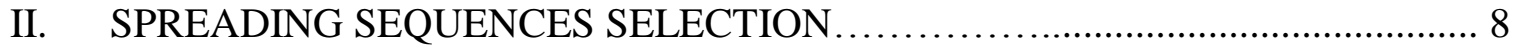

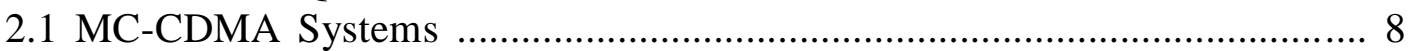

2.2 Multi-path Fading Combating and MAI Mitigation............................... 13

2.3 Simulation Results and Analysis................................................... 15

2.4 Spreading Sequences Selection Conclusions..................................... 19

III. RECEIVER DESIGN FOR MC-CDMA SYSTEMS ................................. 20

3.1 Receiver for Downlink MC-CDMA Systems................................. 20

3.2 Receiver for Uplink MC-CDMA Systems........................................................ 44

IV. INTERFERENCE ANALYSIS FOR MULTICARRIER SYSTEMS.............59

4.1 Performance Analysis of Interference for OFDM Systems ………………...... 59

4.2 Performance Analysis of Interference for MC-CDMA Systems.................. 79

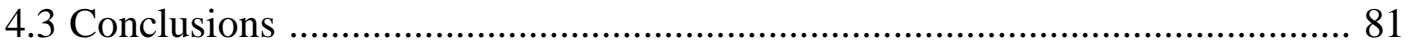

V. ANTI-INTERFERENCE IN MC-CDMA SYSTEMS .............................. 82

5.1 Maximum Likelihood Estimation of Interference Side Information............ 82

5.2 Simulation Results......................................................... 85

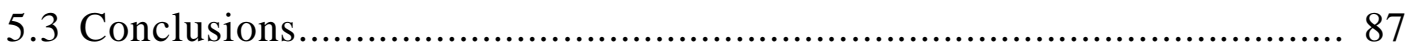

VI. A PRACTICAL MC-CDMA SYSTEM ON SDR PLATFORM................... 88

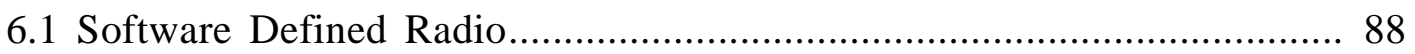

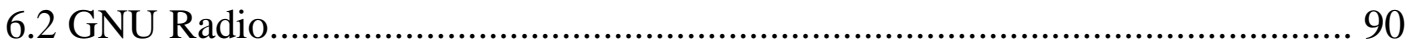

6.3 FIU SDR Platform Project..................................................................... 93

6.4 A Cognitive Radio MC-CDMA System on FIU SDR platform................104

6.5 Experimental Results................................................ 107

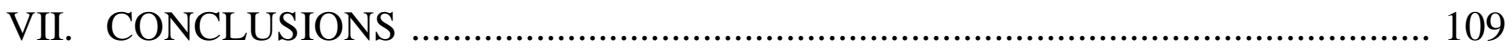

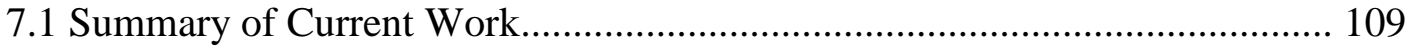

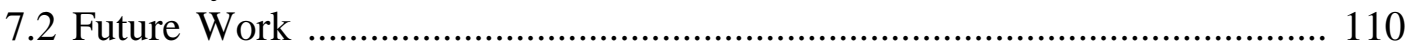

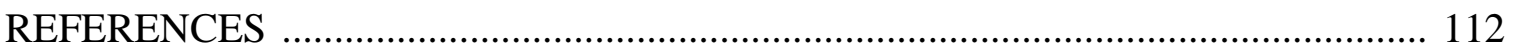

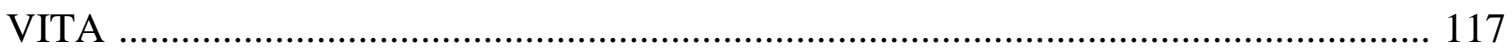




\section{LIST OF TABLES}

TABLE

PAGE

2.1. Main parameters used in simulations ..................................................... 18

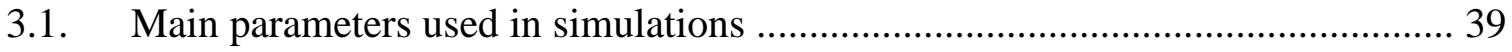

3.2. Main parameters used in simulations ....................................................... 57

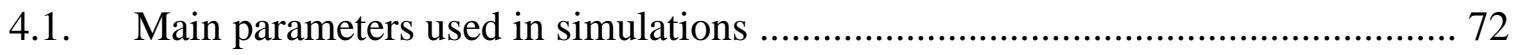

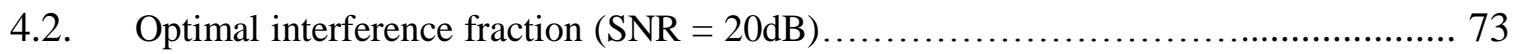

5.1. Interference parameters used in simulations....................................... 85

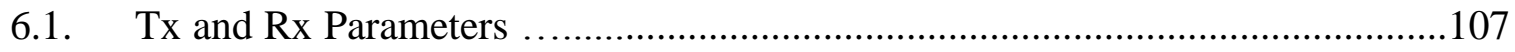




\section{LIST OF FIGURES}

FIGURE

PAGE

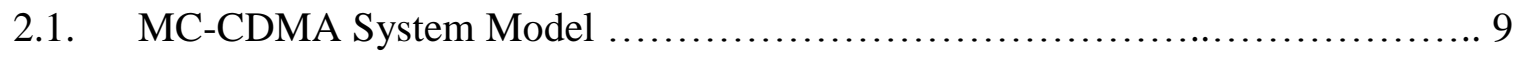

2.2. PAPR comparison of CQS and Walsh Codes........................................ 16

2.3. BER comparison of CQS and Walsh Codes over SUI-2 (SNR: $-19 \mathrm{~dB}) \ldots . .17$

2.4. BER comparison of CQS and Walsh Codes over SUI-4 (SNR: $-19 \mathrm{~dB}) \ldots . .17$

2.5. BER comparison of CQS and Walsh Codes over SUI-6 (SNR: $-19 \mathrm{~dB}$ ).....17

3.1. System model of MC-CDMA downlink system............................ 24

3.2. BER comparison of different receivers under 3GPP TR 25.943 typical urban

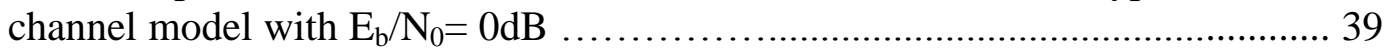

3.3. BER comparison of different receivers under 3GPP TR 25.943 typical rural

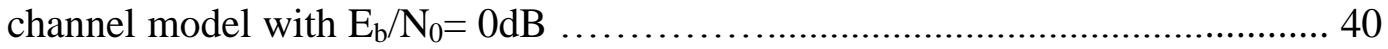

3.4. Channel MSE comparison of full loaded receivers with $\mathrm{Eb} / \mathrm{N} 0=5 \mathrm{~dB} \ldots \ldots \ldots . .42$

3.5. Channel MSE comparison of full loaded receivers with $\mathrm{Eb} / \mathrm{NO}=20 \mathrm{~dB} \ldots \ldots \ldots . .43$

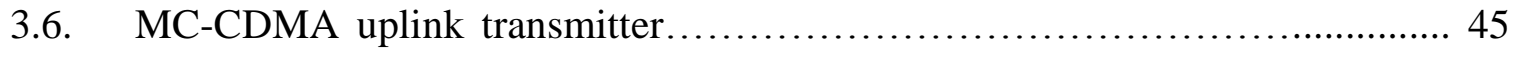

3.7. Channel amplitude PDF.............................................. 47

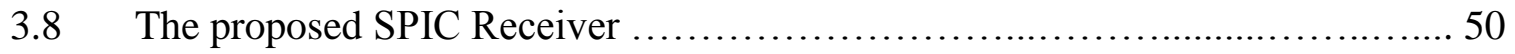

3.9. BER comparison of different receivers under channel SUI-2 ......................... 55

3.10 BER comparison of different receivers under channel SUI-6......................... 55

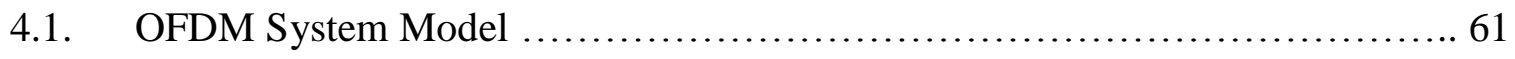

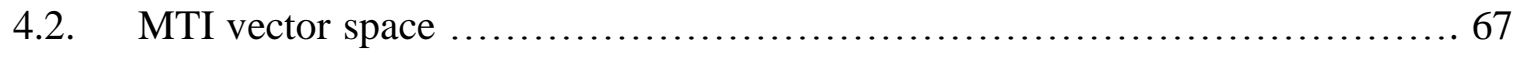

4.3. Comparison between simulation results and theoretical values of all interference strategies (SNR is fixed to $10 \mathrm{~dB}$ ) ........................... 75

4.4. Comparison between optimal-fraction interference and fixed-fraction interference of PBI and MTI (SNR is fixed to $10 \mathrm{~dB}$ ) 
4.5. Comparison of optimal-fraction MTI, optimal-fraction PBI and BNI (SNR is fixed to 10dB in AWGN and Rayleigh channel) ............................ 77

4.6. Comparison of optimal-fraction MTI, optimal-fraction PBI and BNI (SNR is fixed to $10 \mathrm{~dB}$ in Rician channel, $\mathrm{K}=5$ and 10) ................................ 78

5.1. Comparison results of the proposed system with/without interference mitigation policy under the pulse interference $\ldots \ldots \ldots \ldots \ldots \ldots \ldots \ldots \ldots \ldots \ldots . . . \ldots 6$

5.2. Comparison results of the proposed system with/without interference mitigation policy under the partial band interference ........................... 86

5.3. Comparison results of the proposed system with/without interference mitigation policy under the pulse interference and partial band interference...... 87

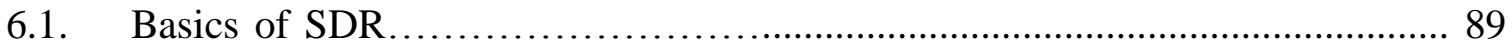

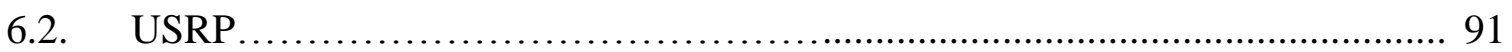

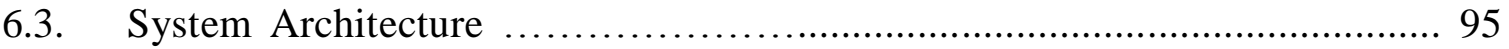

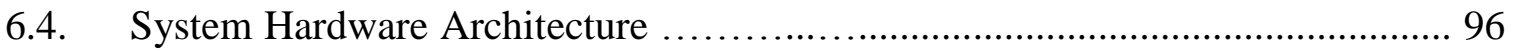

6.5. Proposed SDR Software Architecture........................................................100

6.6. User-centered Design Process ...................................................103

6.7. Spectral Adaptation Waveform ….......................................................105

6.8. Cognitive Radio Network ...............................................................106

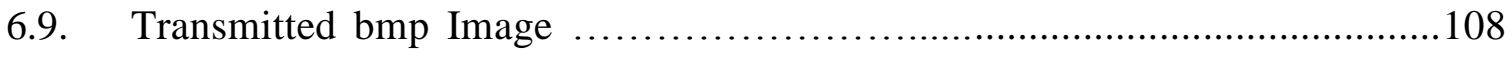

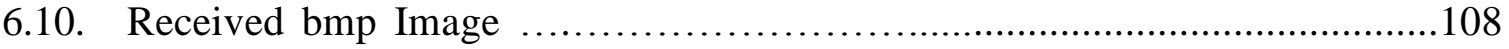




\section{LIST OF FIGURES ABBREVIATIONS}

TERM / UNIT OF MEASUREMENT

Orthogonal Frequency-Division Multiplexing

Inter-Symbol Interference

Multicarrier Code-Division Multiple Access

Bit Error Rate

Low Density Parity-Check Coding

Peak-to-Average Power Ratio

Multiple Access Interference

multi-user detection

interference cancellation

single user detection

parallel interference cancellation

successive interference cancellation

Additive white Gaussian noise

Complex Quadratic Sequences

pilot transmission

data transmission

channel side information

minimum mean square error

Maximal Ratio Combining

log-likelihood ratio

global MMSE
SYMBOL / ABBREVIATION

OFDM

ISI

MC-CDMA

BER

LDPC

PAPR

MAI

MUD

IC

SUD

PIC

SIC

AWGN

CQS

PT

DT

CSI

MMSE

MRC

LLR

GMMSE 
software defined radio

SDR

Phase Shift Keying

PSK

Guard Interval

GI

Inter Carrier Interference

ICI

Stanford University Interim

SUI

Power Spectral Density

PSD

mobile terminal

MT

Least Square

LS

minimum square errors

MSE

time division duplex

TDD

Pre-Orthogonality Restoring Combining

PRE-ORC

probability density function

PDF

signal-to-interference ratio

SIR

quality of service

QoS

quadrature amplitude modulation

QAM

soft-parallel IC

SPIC

partial parallel interference cancellations

PPIC

minimum mean square combining

MMSEC

soft in soft out

SISO

logarithmic maximum a posteriori

Log-MAP

Barrage Noise Interference

BNI

Partial Band Interference

PBI

Multitone Interference

MTI 
Binary Phase Shift Keying $\quad$ BPSK

Differential Binary Phase Shift Keying DBPS

Frequency Domain Differential Demodulation $\quad$ FDDD

Time Domain Differential Demodulation TDDD

$\begin{array}{ll}\text { Line Of Sight LOS } & \text { LOS }\end{array}$

$\begin{array}{ll}\text { signal-to-interference plus noise ratio } & \text { SINR }\end{array}$

Universal Software Radio Peripheral USRP

$\begin{array}{ll}\text { field programmable gate arrays } & \text { FPGA }\end{array}$

noise spectral density $\quad$ NSD

General Purpose Processor $\quad$ GPP

radio frequency $\quad$ RF

$\begin{array}{ll}\text { application-specific integrated circuit } & \text { ASIC }\end{array}$

$\begin{array}{ll}\text { Federal Communication Commission } & \text { FCC }\end{array}$

dynamic frequency selection DFS

$\begin{array}{ll}\text { cyclic redundancy check } & \text { CRC }\end{array}$

Root-Raised Cosine RRC 


\section{CHAPTER I}

\section{INTRODUCTION}

Orthogonal Frequency-Division Multiplexing (OFDM) has been proved to be a promising technology that enables the transmission of higher data rate. The basic idea of OFDM is to use a large number of parallel narrow-band sub-carriers instead of a single wide-band carrier to transport information. With its capability of adapting to severe channel conditions without complex equalization, OFDM can effectively provide broadband wireless communication in hostile multipath environments. Furthermore OFDM is robust against Inter-Symbol Interference (ISI) and fading caused by multipath propagation. Multicarrier Code-Division Multiple Access (MC-CDMA) is a transmission technique which combines the advantages of both OFDM and Code-Division Multiplexing Access (CDMA) [1], so as to allow high transmission rates over severe time-dispersive multi-path channels without the need of a complex receiver implementation. Also MC-CDMA exploits frequency diversity via the different subcarriers, and therefore allows the high code rates systems to achieve good Bit Error Rate (BER) performances. Furthermore, the spreading in the frequency domain makes the time synchronization requirement much less stringent than traditional direct sequence CDMA schemes [2].

Nowadays the forward error-correcting codes are commonly used in wireless communication systems. Recently Low Density Parity-Check Coding (LDPC) has attracted much attentions since it provides a performance which is close to the Shannon limit [3] and linear time complex algorithms for decoding. Some research work has been 
done for LDPC coded MC-CDMA systems. The performance of LDPC coded MCCDMA systems has been given over Rayleigh fading channels in [4]. The effects of subcarrier interleaving on LDPC MC-CDMA systems have been investigated by [5], and in [6] the performance of spread OFDM with LDPC coding in outdoor environments has been discussed. All these works show the great potential of LDPC coded MC-CDMA systems.

\subsection{Problem Statement}

Nothing is perfect. Here comes one major problem of MC-CDMA system: Peak-toAverage Power Ratio (PAPR). High PAPR leads to nonlinear distortion of the amplifier and results in inter-carrier self-interference plus out-of-band radiation. To prevent spectral growth of the transmitted signal, the transmit power amplifier should be operated in its linear region (i.e., so-called input backoff). Large input backoff implies inefficient power conversions and degraded BER. Many approaches have been proposed to solve the PAPR problem in multicarrier systems, which include amplitude clipping, clipping and filtering, coding, tone reservation, tone injection, active constellation extension, and multiple signal representation techniques. All those techniques have their own drawbacks, such as transmit signal power increase, BER increase, data rate loss, computational complexity increase, and so on [7]. On the other hand, suppressing the Multiple Access Interference (MAI) is another crucial problem in the MC-CDMA system. Imperfect cross-correlation characteristics of the spreading codes and the multipath fading make the orthogonality among the users lost, and then cause MAI, which produces serious BER degradation in the system. 
To improve the performance of MC-CDMA systems, multi-user detection (MUD) can be applied. Among them, interference cancellation (IC) methods are fairly promising since they achieve better performance than single user detection (SUD) techniques, while still maintaining a linear complexity in the number of users [8]. The IC for multi-user systems has generally been broken into two categories, parallel and successive. In a downlink MC-CDMA system, the simple and effective power control method is just transmitting all the users' signal with the same power approximately. Under this circumstance, parallel interference cancellation (PIC) is definitely better than successive interference cancellation (SIC) [7] although the complexity of PIC is slightly higher than the SIC. Due to the ability of detecting all the users simultaneously with reduced latency, the PIC is also especially attractive for an uplink MC-CDMA system. Hence the PIC is applied in our work. The concept of SIC/PIC is based on the premise that the received signal can be reliably estimated, which is not always true in a realistic communication system. The main performance limitations of SIC/PIC schemes are: 1) imperfect interference cancellation due to non-ideal channel estimation and 2) error propagation caused by erroneous symbol decision feeding back [9].

As indicated in [10], the techniques for interference suppression in MC-CDMA systems have not been thoroughly explored. So it is necessary to consider this part in our work. 


\subsection{Research Hypotheses}

This dissertation describes the work done with the following hypotheses.

- The frequency selective fading channel has been transformed into multiple flat fading channels for every sub-carrier.

- The proposed MC-CDMA system is quasi-synchronous in uplink side, i.e., the timing of all users is aligned within in a small synchronization window (Within the margin of the cyclic prefix). This assumption is suitable for the reversed link in practice [11].

- The perfect OFDM time and frequency domain synchronization have been assumed in our system.

- Additive white Gaussian noise (AWGN) is used as the noise source in our work.

\subsection{Proposed Approach}

LDPC code is exploited in the MC-CDMA system. The combination of LDPC and spectral diversity of MC-CDMA will improve the system performance and reduce the requirement of coding length. Different from conventional MC-CDMA systems, Complex Quadratic Sequences (CQS) [12] have been applied in the proposed system. CQS has constant envelop in both frequency domain and time domain, and furthermore it is circular auto-orthogonal. These two properties will be very useful to lower PAPR and mitigate the multi-user interference.

The new semi-blind channel estimation and multi-user data detection based on PIC have been proposed. There are two working modes in the system: pilot transmission (PT) mode and data transmission (DT) mode. Periodically, the system enters the PT mode to obtain the channel side information (CSI), then tracks the channel variations and detects 
the actual data in DT mode until next PT mode comes. With a certain period of CSI observations obtained in the PT mode, the variation of the channel is predicted by the short range wireless channel prediction algorithm [13] in the DT mode. With the predicted CSI, the novel data detection based on PIC, minimum mean square error (MMSE) combining and Maximal Ratio Combining (MRC) is employed to achieve the data decision results. The refined CSI is acquired from the decision feedback simultaneously. After analyzing different interference models in multi-carrier OFDM systems, we design the anti-interference techniques in the proposed system. The interference information will be estimated as log-likelihood ratio $(L L R)$. Based on this information, the corresponding anti-interference method is applied.

\subsection{Contributions}

- We solved the problem from the root, i.e., we use CQS instead of Walsh codes because it reduces the PAPR greatly and mitigates MAI effectively as well.

- The noise variance and channel information are not necessary known in advance (The noise is assumed as Gaussian Noise). These parameters can be estimated in the PT mode, and the CSI can be effectively tracked with the short range wireless channel prediction algorithm with low complexity.

- CQS is used as our spreading sequences. Besides its constant envelope property to mitigate the Peak-to-Average Power Ratio (PAPR) in multi-carrier systems, CQS has been proved to be able to eliminate the MAI effectively [5]. Based on that, a new PIC method has been proposed. It is proved that only a small part of active users will interfere with the specified user. By canceling this small portion of users it is enough to get rid of all MAI in the ideal case, and the complexity is reduced significantly. 
Also along with the decreasing of the cancelled user number, the errors brought by imperfect channel estimation and erroneous data detections are mitigated as well. Thus it improves the performance and decreases the complexity at the same time.

- The Combination of MMSE and global MMSE (GMMSE) is adopted and quite good results are obtained with minimum efforts. Unlike with the GMMSE method, which calls for the solution of $N \times N$ linear equations, here only $G \times G$ linear equations need to be solved, where $\mathrm{N}$ is FFT size and G is the fraction of the current active user number which is equal to (current active users number)/5 in this paper. This greatly reduces the complexity.

- In uplink, we apply the same policy. However, since the channel estimation has been done in mobile terminals, the pre-equalization is exploited in the MT. This greatly reduced the complexity of Base Station without degrading the performance.

- The comprehensive study of interference in multi-carrier systems has been given. The analysis of multiple tone interference (MTI) is given for the first time, and the simulation results prove its correctness.

- Based on the properties of CQS constant envelope, a new anti-interference method has been developed for MC-CDMA systems.

\subsection{Dissertation Organization}

The dissertation is organized as follows. Chapter II includes a discussion of spreading sequences selection and introduces the CQS based LDPC MC-CDMA system. Chapter III presents the receiver design both in downlink and uplink. Chapter IV analyzes the interference models in multi-carrier systems. Chapter V discusses the anti-interference in 
the proposed system. Chapter VI gives one practical application of the proposed system on the software defined radio (SDR) platform. Chapter VII draws the conclusions and lists future research directions. 


\section{CHAPTER II}

\section{SPREADING SEQUENCES SELECTION}

In this chapter, the selection of spreading sequences for LDPC coded MC-CDMA systems is considered. Our aim is to jointly minimize the MAI and the PAPR. The PAPR is used for the evaluation of the dynamic range of the transmitted Phase Shift Keying (PSK) modulated multicarrier signal envelope for the orthogonal spreading sequences candidates and the MAI elimination effects are tested with the simple MRC receiver.

\subsection{MC-CDMA Systems}

The overview of the system model is illustrated in Figure 2.1. First, the input data passes through a LDPC encoder. LDPC codes belong to linear block codes defined by a very sparse parity-check matrix $H_{L}$ with dimensions $M_{L} \times N_{L}$. A LDPC code is called regular if its parity-check matrix $H_{L}$ has constant non-zero entries in its columns and constant non-zero entries in its rows; otherwise it is an irregular LDPC code. In this work, we use irregular LDPC codes. Note that the code rate of the LDPC is $R_{L}=K_{L} / N_{L}$, where $K_{L}=N_{L}-M_{L}$. After the channel coding, the data is modulated by a BPSK modulator, then converted into a parallel form. The system in the paper employs $N$ sub-carriers. Every one of the user bits will be duplicated $N$ times. Then all the ${ }_{N}$ bits are spread by the CQS. The CQS has many interesting properties, to which we will give a brief introduction. The CQS is defined as follows: 


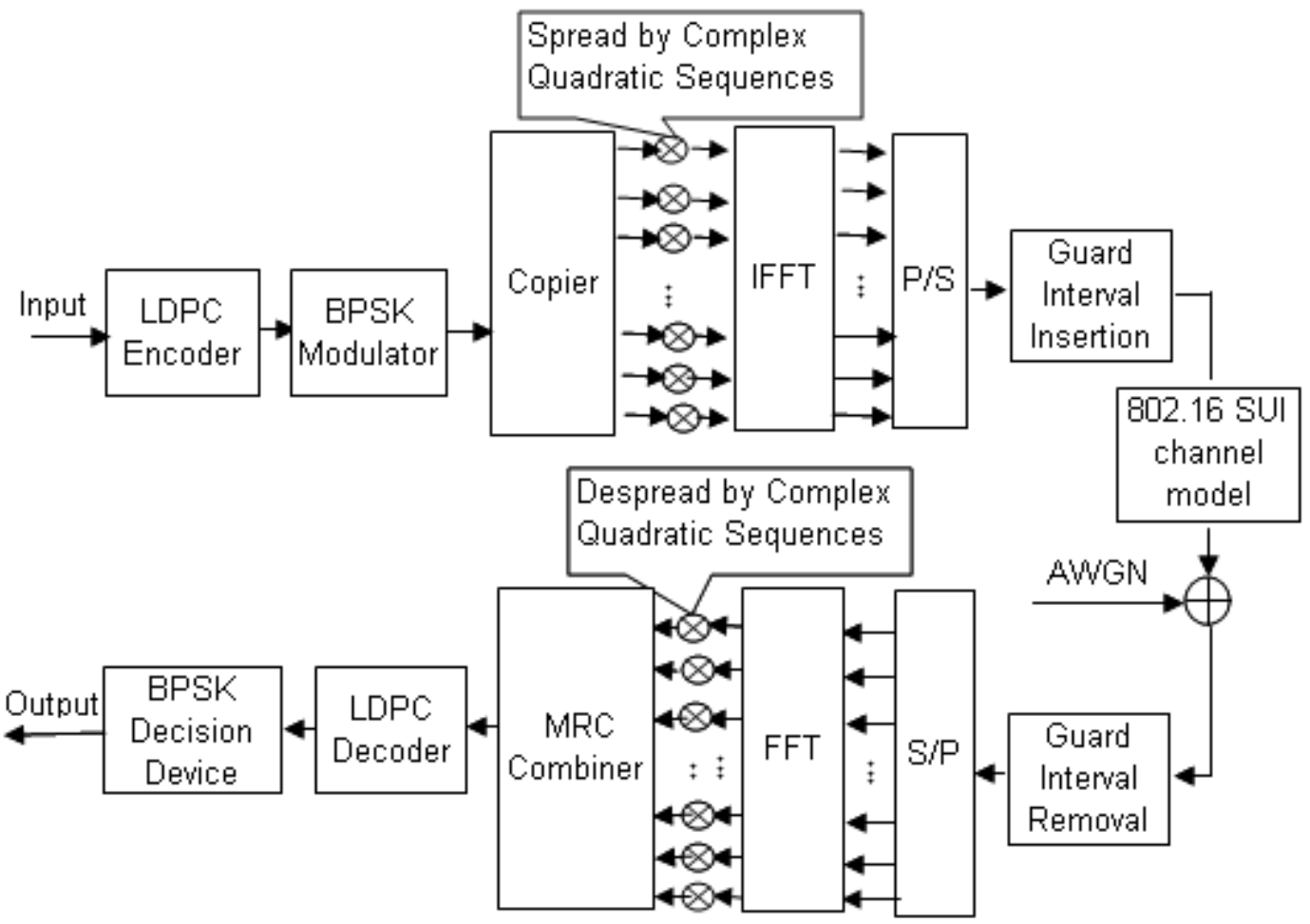

Figure 2.1. MC-CDMA System Model

$$
\begin{aligned}
g_{n} & =e^{-j \frac{\pi}{8}} e^{j \frac{2 \pi 1}{N} n^{2}} / N, & n & =0,1, \ldots, N-1 \\
G_{k} & =e^{j \frac{\pi}{8}} e^{-j \frac{2 \pi 1}{N} k^{2}} / \sqrt{N}, & k & =0,1, \ldots, N-1
\end{aligned}
$$

where $\left\{g_{n}\right\}$ and $\left\{G_{k}\right\}$ are a DFT/IDFT pair, and $G_{n}^{*}=\sqrt{N} g_{n}$. It can be noticed that both the time domain sequences $\left\{g_{n}\right\}$ and the frequency domain sequences $\left\{G_{k}\right\}$ have a constant envelope. If the modulator is $M$-PSK type, this implies that the transmitted signal has a constant envelope in both the time and frequency domains. With the transmitted signal $S(t)$, the definition of PAPR in one OFDM symbol duration $\mathrm{T}$ is

$$
\operatorname{PAPR}(S(t))=\frac{\max _{0 \leq t \leq T}|S(t)|^{2}}{E\left\{|S(t)|^{2}\right\}}
$$


Clearly the constant envelope property of CQS eliminates the PAPR problem completely in a single user case. Even in a multi-user environment the PAPR also can be reduced greatly. Another important property has been proved [12] that both the sequences $G_{k}$ and $g_{n}$ are circular auto-orthogonal. This property is very useful for MUD and combating multi-path fading, which will be discussed in detail later. As usual for multi-carrier systems, Guard Interval (GI) has been added to suppress the Inter Symbol Interference (ISI) and the Inter Carrier Interference (ICI) after IFFT transformation. To simplify the analysis, we will ignore the parts of GI insertion and removal from now on. Since only the downlink system is necessary to be considered (The uplink system can be regarded as a quasi-synchronous one, so the same analysis works under this situation), multiple users will send signals at the same time. Assuming there are $M$ users in the communication link, the transmitted signal of the $k$-th sub-carrier can be represented as

$$
X_{k}=\sum_{m=0}^{M-1} b_{(m)} G_{(m), k} \quad k=0,1 \ldots, N-1
$$

where $b_{(m)}$ is the current message symbol of user $m$ after processing of the modulator (For BPSK, $b_{(m)} \in\{ \pm 1\}$ ), and $\left\{G_{(m), 0}, G_{(m), 1}, \ldots, G_{(m), N-1}\right\}$ are the $m$-th user's frequency spreading sequences. The spreading sequences for different users should be mutually orthogonal. The set of spreading sequences for all the users is generated by the following equation

$$
G_{(m), k}=e^{-j \frac{2 \pi}{N} k m} G_{k} \quad k=0,1, \ldots, N-1
$$

Due to the circular auto-orthogonal property of CQS, we can get at most $N$ different orthogonal spreading sequences to support $N$ different users, i.e. $M \leq N$ for the proposed 
system. To simplify the notations we define two vectors as: $\mathbf{X}=\left(X_{0}, X_{1}, \ldots, X_{N-1}\right)^{T}$ and $\mathbf{G}_{(\mathbf{m})}=\left(G_{(m), 0}, \ldots, G_{(m), N-1}\right)^{T}$, then Eqn. (2.3) can be written as:

$$
\mathbf{X}=\sum_{m=0}^{M-1} b^{(m)} \mathbf{G}_{(\mathbf{m})}
$$

The transmitted signal is sent across a multi-path fading channel model: modified 802.16 Stanford University Interim (SUI) channel model [14]. Because SUI is developed for fixed wireless applications, we have made some modifications to fit the mobile applications. In the fixed wireless channels the Doppler Power Spectral Density (PSD) of the scatter component is mainly distributed around $f=0 \mathrm{~Hz}$, which is different from the mobile channels. Hence the classical Jake's spectrum is applied in this paper instead of the Doppler Spectrum model used in [14]. Here we assume all multipath components have the same Doppler spectrum, which has the form

$$
S_{d}(f)= \begin{cases}\frac{1}{\pi f_{d}} \frac{1}{\sqrt{1-\left(\frac{f}{f_{d}}\right)}}, & |f|<\left|f_{d}\right| \\ 0 & \end{cases}
$$

where $f_{d}$ is the maximum Doppler shift.

On the terminal side, after the removal of the GI, the received signal is transferred from a serial stream into $N$ parallel streams. Then the data is processed by the FFT and CQS dispreading unit. After these two steps, the received data of the $l$-th user is

$$
\mathbf{Y}_{(l)}=\left(\mathbf{H}_{(l)} \mathbf{X}+\mathbf{W}\right) \cdot \mathbf{G}_{(l)}^{*}
$$

where "." denotes entry-by-entry product of two vectors and $(\cdot)^{*}$ indicates the complex conjugation. In this equation, $\mathbf{H}_{(l)}=\operatorname{diag}\left(H_{(l), 0}, H_{(l), 1}, \ldots, H_{(l), N-1}\right)^{T}$ gives the frequency domain channel attenuations of the $l$-th user and $\mathbf{W}$ is the vector of the uncorrelated 
complex frequency domain Gaussian random noises with variance $\sigma^{2}$. Assuming there is perfect Channel Side Information (CSI) on the receiver side, the Maximal Ratio Combining (MRC) can be used in the proposed LDPC coded MC-CDMA system. Let $\mathbf{Q}_{(l)}=\left\{Q_{(l), 0}, \ldots, Q_{(l), N-1}\right\}^{T}$ stands for the $l$-th user's corresponding equalization gain vector, given as

$$
\mathbf{Q}_{(l)}=\frac{N}{\sum_{t=0}^{N-1}\left|H_{(l), t}\right|^{2}}\left(H_{(l), 0}^{*}, H_{(l),}^{*}, H_{(l), 2}^{*}, \ldots, H_{(l), N-1}^{*}\right)^{T}
$$

The output of MRC combiner can be expressed as

$$
r_{(l)}=\left(\mathbf{Q}_{(l)}\right)^{T} \mathbf{Y}_{(l)}
$$

The MRC only tries to increase the SNR and does nothing to restore the orthogonality among the users. However, CQS can reduce the MAI of the system effectively, so MRC can be used here as a simple method. Furthermore the pre-equalization techniques are possible to help the system restore the orthogonality in the future. The result scalar $r_{(l)}$ will be demodulated, and decoded by the LDPC decoder to get the transmitted data. The log domain decoding algorithm for LDPC codes is based on the belief propagation theory and it is similar to that in [15]. The interference term in (2.9) can be written as

$$
I_{(l)}=\frac{\left.\left.N \sum_{k=0}^{N-1}|| H_{(l), k}\right|^{2}\left(\sum_{\substack{m=0, m \neq l}}^{M-1} b^{(m)} G_{(m), k}\right)+W_{k} H_{(l), k}^{*}\right) G_{(l), k}^{*}}{\sum_{t=0}^{N-1}\left|H_{(l), t}\right|^{2}}
$$

where $W_{k}$ is the frequency domain Gaussian random noise with variance $\sigma^{2}$. Assuming that $N$ is large (This is the general case in most MC-CDMA systems), the interference 
$I_{(l)}$ can be modeled as a Gaussian random variable (Central Limit Theorem) with zero mean, and the variance of $I_{(l)}$ is given by [16]

$$
\begin{aligned}
& \sigma_{I}^{2}=E\left\{I_{(l)} \cdot I_{(l)}^{*}\right\} \\
& =\frac{N \sum_{k=0}^{N-1}\left(\left|H_{(l), k}\right|^{4} \frac{M-1}{N}+\sigma^{2}\left|H_{(l), k}\right|^{2}\right)}{\left(\sum_{t=0}^{N-1}\left|H_{(l), t}\right|^{2}\right)^{2}}
\end{aligned}
$$

where $H_{(l), k}$ is assumed to be time-invariant in one LDPC block. Since only the real part of this interference is effective for the BPSK decision device, the real part variance becomes

$$
\sigma_{\text {real }}^{2}=\frac{1}{2} \sigma_{I}^{2}
$$

Thus, the $L L R$ of the Channel is

$$
L\left(P_{l}\right)=\log \frac{P_{l}^{0}}{P_{l}^{1}}=-\frac{2 \cdot \operatorname{REAL}\left(r_{(l)}\right)}{\sigma_{\text {real }}^{2}}
$$

where $\operatorname{REAL}(\cdot)$ function returns the real part of the input. Eqn. (2.13) will be used as the initial a priori $L L R$ value of the LDPC decoder.

\subsection{Multi-path Fading Combating and MAI Mitigation}

As mentioned before, the CQS is very useful for combating multi-path fading. Eqn. (2.4) gives the frequency domain spreading sequences for a specific user. We can get corresponding sequences in the time domain as

$$
g_{(m), k}=g_{(k-m)_{N}} \quad k=0,1, \ldots, N-1
$$

where $g_{(k-m)_{N}}$ represents a right cyclic shift by $\mathrm{m}$ of the basic CQS $g_{k}$ in the base of $N$. When the signal goes through a multi-path channel, the received signal is constructed by 
the original signal and the delayed ones. Because all the delayed sequences are orthogonal with each other, it is quite easy to separate different paths by correlating the received sequence with the cyclic shifted sequences of $g_{k}[12]$. Hence in a deep fading multi-path channel, much interference caused by delayed paths can be eliminated by the CQS without any extra complexity. This will improve the system's performance significantly. On the other hand, the CQS can also mitigate MAI effectively. Eqn. (2.10) can be rewritten as

$$
\begin{aligned}
& =\frac{N \sum_{\substack{m=0 \\
m \neq l}}^{M-1 N-1}\left|H_{(l), k}\right|^{2} \frac{b^{(m)}}{N} e^{j \frac{2 \pi}{N} k(l-m)}}{\sum_{t=0}^{N-1}\left|H_{(l), t}\right|^{2}} \quad \rightrightarrows M A I \\
& +\frac{N \sum_{k=0}^{N-1} H_{(l), k}^{*} W_{k} e^{j \frac{2 \pi}{N} k l} G_{k}^{*}}{\sum_{t=0}^{N-1}\left|H_{(l), t}\right|^{2}} \quad \text { Woise }
\end{aligned}
$$

For MAI term only, applying the Plancherel theorem [17], we get

$$
I_{(l), M A I}=\frac{N \sum_{\substack{m=0 \\ m \neq l}}^{M-1} \sum_{n=0}^{N-1} h_{n}^{*} h_{(n-m+l)_{N}} b_{(m)}}{\sum_{t=0}^{N-1}\left|H_{(l), t}\right|^{2}}
$$

where $h_{n}$ is the time domain channel impulse response. Now it is assumed that the maximum delay spread of a multi-path fading channel is $L(N>L)$, i.e., $h_{n}=0$ when $n>L$. It can be found that when $( \pm(m-l))_{N}>L$ (The “" \pm " signifies both right and left directions, so it represents the cyclic shift distance between two users is larger than $L$ ), then $\sum_{n=0}^{N-1} h_{n}^{*} h_{(n-m+l)_{N}}=0$. In other words, when the cyclic shift distance between two users is larger than the maximum delay spread of a multi-path fading channel $L$, there will be 
no MAI between them at all. Obviously in a full loaded MC-CDMA downlink system, the maximum number of users which can cause interference is reduced from $N-1$ to $2 L$ for every user. For a low loaded system, if we arrange the cyclic shift distances among all the active users in a smart way (The distances are all larger than $L$ ), it is possible to eliminate MAI completely. Therefore Eqn. (2.11) can be modified as

$$
\begin{aligned}
& \sigma_{I}^{2}=E\left\{I_{(l)} \cdot I_{(l)}^{*}\right\} \\
& =\frac{N \sum_{k=0}^{N-1}\left(\left|H_{(l), k}\right|^{4} \frac{U}{N}+\sigma^{2}\left|H_{(l), k}\right|^{2}\right)}{\left(\sum_{t=0}^{N-1}\left|H_{(l), t}\right|^{2}\right)^{2}}
\end{aligned}
$$

where $U$ represents the number of users which can cause interference to the $l$ th user. The $U$ can be found just by counting the number of active users, which have the cyclic distances no larger than $L$ to the $l$ th user. The data area of $U$ is from 0 to $2 L$, and in a low loaded system it can be fixed as 0 (no MAI). This shows the great potential of the proposed system to reduce the complexity of MUD.

\subsection{Simulation Results and Analysis}

The PAPR and BER performance of different spreading sequences for MC-CDMA downlink system is evaluated by the means of the software simulation. Based on the 802.16 standard [18], the main parameters used in the simulation are summarized as Table 2.1. In this table, to simplify the problem, we use 256 as the number of data subcarriers instead of 200 in the 802.16 standard. In the simulation, the Walsh codes will be used in the system as a reference since it is clear that orthogonal spreading sequences (Orthogonal Gold and Walsh codes) perform better than non-orthogonal sequences (Gold 


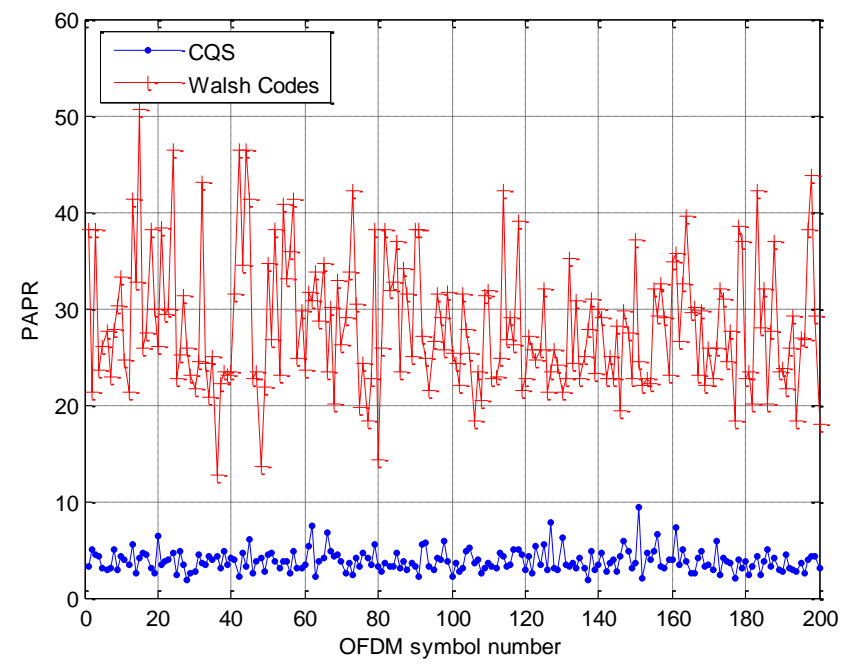

Figure 2.2. PAPR comparison of CQS and Walsh Codes

sequences) and the Walsh codes perform the best among them (Orthogonal Gold, Walsh codes and Gold sequences) in a downlink MC-CDMA system [19].

In the PAPR test scenario, 200 OFDM symbols are transmitted. Every symbol includes the data of multiple users. The number of users for every symbol is an equally distributed random variable varied from 10 to 20 . Both CQS and Walsh codes have been tested. Figure 2.2 shows the simulation results. Because of the constant envelope property of the CQS in the time domain it is obvious that the CQS offers an overwhelming advantage over the Walsh codes. In the following BER performance simulations, the BER degradation caused by PAPR is ignored. Even though this gives more benefits to the Walsh codes in the comparison, we will show that the CQS still performs better than the Walsh codes.

In the following simulations, the performance of CQS and Walsh codes in a multi-user environment over different SUI channel models is compared. There are six omnidirectional antenna channel models which appear in [14]. From all of them, we select 

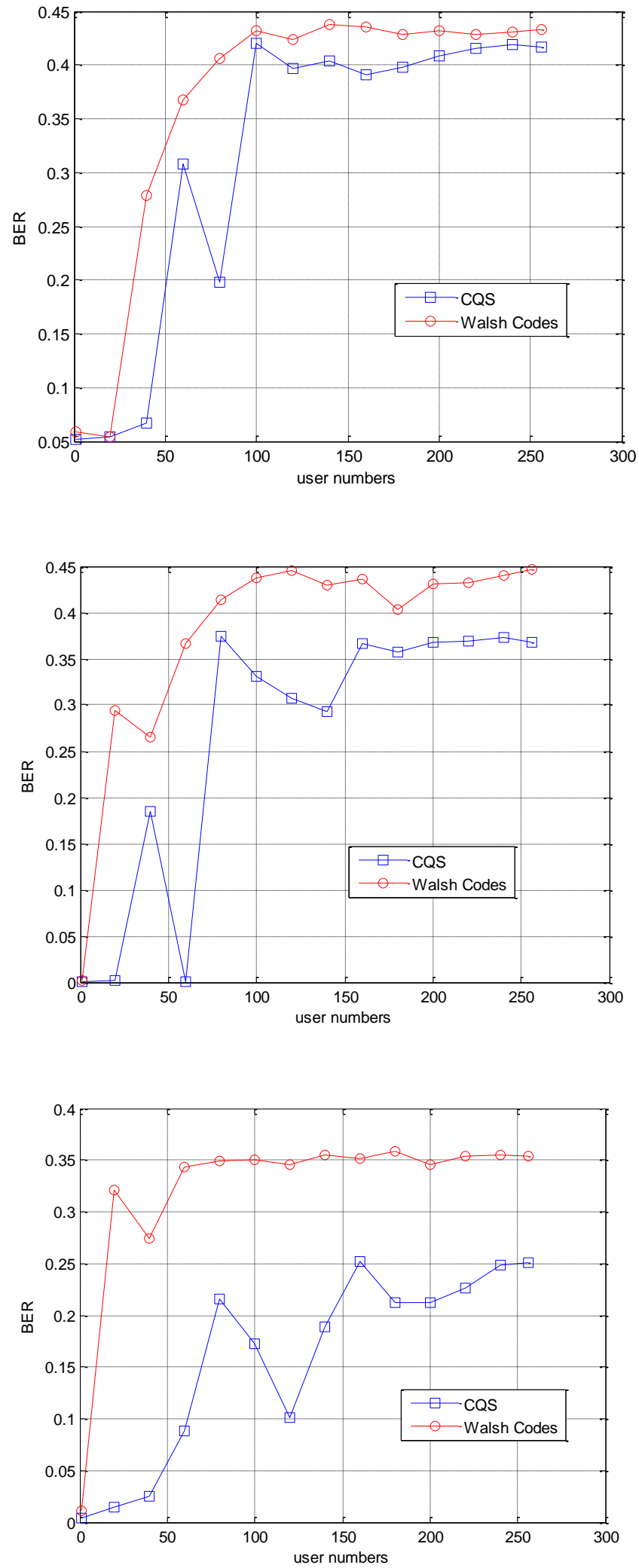

Figure 2.3. BER comparison of CQS and Walsh Codes over SUI-2 (SNR: -19 dB)
Figure 2.4. BER comparison of CQS and Walsh Codes over SUI-4 (SNR: -19 dB)

Figure 2.5. BER comparison of CQS and Walsh Codes over SUI-6 (SNR: -19dB) 
three as our test channels: SUI-2, SUI-4 and SUI-6. These three channel models cover three most common terrain categories found across the United States. SUI-2 has low delay spread; SUI-4 has moderate delay spread, and SUI-6 has high delay spread. Since the LDPC coded MC-CDMA system has quite good performance in SUI channel models, low SNR is used to avoid trivial BERs. The simulation results are shown in Figure 2.3, 2.4 and 2.5 separately. All three figures show that the CQS has better performance over the Walsh codes. In the low delay spread case (SUI-2), when the number of users is 40 , the BER of the CQS is 0.0668 , while the Walsh code drives the BER to 0.2788. It is found that the dominant part of SUI-2's channel impulse response is within the length of 6. While the cyclic shift distances among all the users can be set larger than or equal to 6 with the user number of 40 , so the MAI has been mitigated by CQS according to the argument of Section 2.2. With the increase of user number, more and more users enter the region of " $2 L$ " length part, in which the other users can cause interference to the detected user. Therefore the BER of CQS also increases. In spite of this, the CQS still overperforms Walsh codes when the user number $\geq 40$. In higher delay spread cases (SUI-4 and SUI-6), the advantages of the CQS are more obvious in Figure 2.4 and Figure 2.5 compared with low delay spread case (SUI-2). The CQS can effectively mitigate the

Table 2.1: Main parameters used in simulations

\begin{tabular}{|c|c||c|c|}
\hline Signal bit rate & $4 \mathrm{MHz}$ & $\begin{array}{c}\text { Modulation } \\
\text { scheme }\end{array}$ & BPSK \\
\hline $\begin{array}{c}\text { Number of } \\
\text { Data sub- } \\
\text { carriers }\end{array}$ & 256 & Cyclic prefix & 16 us \\
\hline FFT length & 256 & Channel model & $\begin{array}{c}\text { Modified SUI } \\
\text { with AWGN }\end{array}$ \\
\hline $\begin{array}{c}\text { Maximum } \\
\text { Doppler } \\
\text { Frequency }\end{array}$ & $10 \mathrm{~Hz}$ & $\begin{array}{c}\text { OFDM symbol } \\
\text { period }\end{array}$ & 64 us \\
\hline $\begin{array}{c}\text { Spreading } \\
\text { Sequences }\end{array}$ & $\begin{array}{c}\text { CQS } \\
\text { Walsh codes }\end{array}$ & LDPC code rate & 0.8 \\
\hline
\end{tabular}


BER degradation brought by Multipath delay spread. That's the reason why the CQS has much better performance in higher delay spread cases. Based on these results, it is found that the CQS gives an overall advantage over Walsh codes, no matter if the channel conditions are high or low delay spread, and the system is low or high loaded.

\subsection{Spreading Sequences Selection Conclusions}

In this chapter, a LDPC Coded MC-CDMA downlink system with constant envelope spread sequences has been proposed. The CQS is used in our system. It is shown that the CQS can reduce the PAPR effectively because of its constant envelope property. Also the CQS can mitigate MAI considerably. In a traditional LDPC Coded MC-CDMA system, the number of interference users is always equal to $M-1$, where $M$ is the number of current active users. While in the proposed system there will only be $2 L$ users contributing to MAI at most ( $L$ is the maximum delay spread of a multi-path fading channel). Hence for a full loaded system, this means a big decrease for MAI. For a low loaded system, the MAI can be eliminated completely by making the cyclic shift distances among all the users larger than $L$. From analysis and simulation results, it is shown that the proposed system outperforms the conventional LDPC coded MC-CDMA system in both PAPR reduction and BER performance in a multi-user environment. In a word, the proposed system can work well in various loading situations. As in the spreading sequences selection for uplink and downlink MC-CDMA systems, PAPR and MAI minimization are two very important issues [20], it is reasonable to believe that the CQS is a good choice for MC-CDMA system, not only for the downlink, but also for the uplink system. 


\section{CHAPTER III}

\section{RECEIVER DESGIN FOR MC-CDMA SYSTEMS}

In this chapter, we discuss the receiver design for downlink and uplink MC-CDMA systems. The downlink receiver model is given in the first part, and then the research results are extended to the uplink.

\subsection{Receiver for Downlink MC-CDMA Systems}

To improve the performance of MC-CDMA systems, multi-user detections (MUD) are applied and interference cancellation (IC) methods are fairly promising. They achieve better performance than single user detection (SUD) techniques and still maintain a linear complexity in the number of users [8]. In general, the IC methods for multi-user systems are broken into two categories: parallel and successive. In a downlink MC-CDMA system, the simple and effective power control method is to transmit the signals of all the users with the same power approximately. Under this circumstance, parallel interference cancellation (PIC) is definitely better than successive interference cancellation (SIC) [7]. Accordingly we decide to use PIC even though its complexity is slightly higher than that of SIC. The concept of SIC/PIC is based on the premise that the received signal can be reliably estimated, which is not always true in a realistic communication system. As indicated in [9], the main limitations of SIC/PIC schemes are: 1) imperfect interference cancellation due to non-ideal channel estimation and 2) error propagation caused by erroneous symbol decision feeding back. 
Channel estimation is a crucial part of the receiver structure. Since it is obvious that the blind channel estimation is disadvantageous in mobile applications, the channel estimation is generally based on the use of pilot subcarriers in given positions of the frequency-time grid. To ensure adequate estimation accuracy in rapid time-varying channels, it is suggested that the pilot subcarriers be placed in each OFDM symbol [21]. However, long spreading codes can not be used in order to maintain the orthogonality and hence the number of maximum active users is very limited. Additionally, the effect of interpolation is not so well for a rapid time-varying fading channel even a complicated interpolation method is applied.

Many receiver design frameworks need to know the noise variance and channel covariance matrix, which are usually hard to obtain in practice for a mobile terminal. Hence, the channel estimation and data decision results should be extracted without all this information. For the mobile terminal (MT), the complexity reduction is a critical task. We try to solve all the problems in a low complexity way.

Here, we propose a unique low complexity semi-blind channel estimation and data detection scheme for downlink MC-CDMA systems. There are two working modes in the system: pilot transmission (PT) mode and data transmission (DT) mode. Periodically, the system enters the PT mode to obtain the channel side information (CSI), then tracks the channel variations and detects the actual data in DT mode until the next PT mode comes. With a certain period of CSI observations obtained in the PT mode, the variation of the channel is predicted by the short range wireless channel prediction algorithm [13] in DT mode. With the predicted CSI, the novel data detection based on PIC, minimum mean square error (MMSE) and maximal ratio combining (MRC) is employed to achieve the 
data decision results. The refined CSI is acquired from the decision feedback simultaneously. The process may be repeated several times until the CSI converges. The final CSI values are stored as the basis of the next CSI prediction. The noise variance is estimated in the PT mode. It is reasonable that we assume it is unchanged during the whole DT mode period.

In our work, we focus on a joint semi-blind channel estimation and multi-user detection receiver for downlink MC-CDMA systems. The contributions are listed as follows.

1. The noise variance and channel information are not necessarily known in advance. (The noise is assumed as Gaussian Noise.) The parameters can be estimated in PT mode, and the CSI can be effectively tracked with the short range wireless channel prediction algorithm [13] in DT mode with low complexity.

2. The Combination of MMSE and global MMSE (GMMSE) is adopted and quite good results are obtained with minimum efforts. Unlike with the GMMSE method, which calls for the solution of $N \times N$ linear equations, here only $G \times G$ linear equations need to be solved, where $N$ is FFT size and $G$ is the fraction of the current active user number which is equal to (current active users number)/5 in this paper. This greatly reduces the complexity.

3. Complex Quadratic Sequences (CQS) [13] is used as our spreading sequences. Besides its constant envelope property to mitigate the Peak-to-Average Power Ratio (PAPR) in multi-carrier systems, CQS has been proved to be able to eliminate the MAI effectively in [13]. Based on that, a new PIC method has been proposed. It is proved that only a small part of active users will interfere with the specified user. By canceling this small portion of users it is enough to get rid of all MAI in the ideal 
case, and the complexity is reduced significantly. Also along with the decreasing of the cancelled user number, the errors brought by imperfect channel estimation and erroneous data detections are mitigated as well. Thus it improves the performance and lowers the complexity at the same time.

The channel encoder and decoder are omitted for the sake of simplifying the system model. They can be added to improve the system performance further. In a downlink MC-CDMA system, we do not apply the design, in which the decoder is involved in the PIC cancellation process as shown in [22] and [23], since it is not feasible to implement this kind of processing on a MT due to its complexity.

Under different channel and system loading conditions, the simulation results and analysis indicate that our proposed system has good BER performance and channel tracking ability.

\subsubsection{System Description}

The overview of the system model is illustrated in Figure 3.1. The channel encoder and decoder will not change the essence of the problem, so we ignore them. The system employs $N$ sub-carriers. Every one of the user bits will be duplicated $N$ times, or synonymously, the spreading factor equals to 1 . Then all the $N$ bits are spread by the CQS. As usual for multi-carrier systems, Guard Interval (GI) has been added to suppress the ISI and the ICI after IFFT transformation. However, to simplify the analysis, we will ignore the parts of GI insertion and removal from now on. Furthermore, the perfect OFDM time and frequency domain synchronization have been assumed in our system as well. Since this is a downlink system, multiple users will send signals at the same time. 


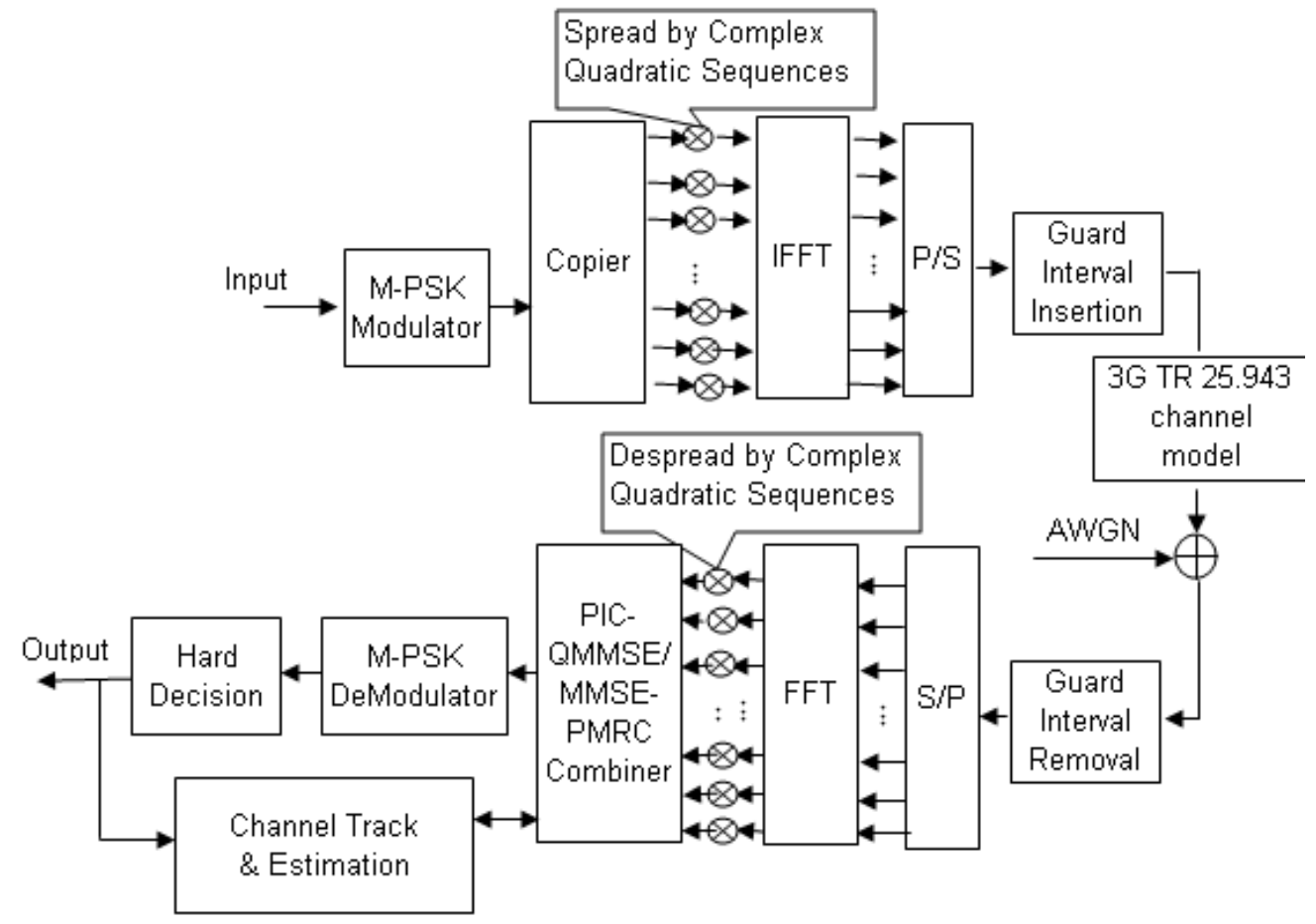

Figure 3.1. System model of MC-CDMA downlink system

Assuming there are $M$ users in the communication link and the base station (BS) transmits with normalized equal power towards all users, the transmitted signal of the $k$ th sub-carrier can be represented as

$$
X_{k}=\sum_{m \in \Theta_{s}} b_{(m)} G_{(m), k} \quad k=0,1, \ldots, N-1
$$

where $\Theta_{s}$ is made of $M$ different numbers arbitrarily selected from $0 \sim \mathrm{N}-1$, representing the ordered index set that forms a mapping from the active users to its corresponding spreading sequences, $b_{(m)}$ is the current message symbol of user $m$ after processing of the modulator (For M-PSK, $\left|b_{(m)}\right|=1$ ), and $\left\{G_{(m), 0}, G_{(m), 1}, \ldots, G_{(m), N-1}\right\}$ are the $m$-th user's frequency spreading sequences. The set of spreading sequences for all the users is generated by the following equation 


$$
G_{(m), k}=e^{-j \frac{2 \pi}{N} k m} G_{k} \quad k=0,1, \ldots, N-1
$$

To simplify the notations we define two vectors as: $\mathbf{X}=\left(X_{0}, X_{1}, \ldots, X_{N-1}\right)^{T}$ and $\mathbf{G}_{(\mathbf{m})}=\left(G_{(m), 0}, \ldots, G_{(m), N-1}\right)^{T}$, then $(3.1)$ can be rewritten as:

$$
\mathbf{X}=\sum_{m \in \Theta_{s}} b_{(m)} \mathbf{G}_{(\mathbf{m})}
$$

The transmitted signal is sent across a tapped-delay multi-path fading channel. Approximating the channel paths as a finite number of $L$ distinct paths, the impulse response for the radio channel may be written as:

$$
h(\tau)=\sum_{i=0}^{L-1} a_{i}(\tau) \delta\left(\tau-\tau_{i}\right)
$$

where $a_{i}(\tau)$ is the path gain of path $i$ and $\tau_{i}$ is the path delay of path $i$. Here we assume all multipath components have the same Doppler spectrum, which has the form as

$$
S_{d}(f)=\left\{\begin{array}{l}
\frac{1}{\pi f_{d}} \frac{1}{\sqrt{1-\left(\frac{f}{f_{d}}\right)}}, \quad|f|<\left|f_{d}\right| \\
0
\end{array}\right.
$$

where $f_{d}$ is the maximum Doppler shift.

There are two working modes for the transmitter: pilot transmission (PT) mode and data transmission (DT) mode. Periodically, the transmitter enters the PT mode to obtain the CSI, then tracks the channel variations and transmits actual data until next PT mode period starts.

\section{A. PT Mode}

In the PT mode, only pilots are transmitted. The pilot symbol is represented as

$$
\mathbf{X}_{\mathbf{p}}=g_{p} \mathbf{G}_{(256)}
$$


where $g_{p}$ is the pilot gain for achieving more precise channel information and $\mathbf{G}_{(25)}$ is the pilot specific spreading code. This spreading code can be chosen from the set of spreading sequences arbitrarily. The fixed value is employed only for the sake of the clear illustrations.

The received signal can be written as

$$
\mathbf{Y}_{\mathbf{p}}=\mathbf{X}_{\mathbf{p}} \cdot \mathbf{H}_{\mathbf{p}}+\mathbf{W}_{\mathbf{p}}
$$

where $\quad \mathbf{H}_{\mathbf{p}}=\operatorname{diag}\left(H_{p, 0}, H_{p, 1}, H_{p, 2}, \ldots, H_{p, N-1}\right) \quad$ gives the frequency domain channel attenuations in the PT mode and $\mathbf{w}_{\mathbf{p}}$ is the vector of the uncorrelated complex frequency domain Gaussian random noise. Simple Least Square (LS) can be applied to estimate the $\mathbf{H}_{\mathbf{p}}$. However, in the proposed method, the channel tracking ability highly depends on the accuracy of the results during the PT period. Hence a more advanced method is necessary. Many methods have been proposed to improve the performance of the LS method, such as LMMSE [24], SVD based on LMMSE [25], Low Rank [26] etc. All those methods are quite complicated and they all need the channel autocorrelation and noise variance, which are generally unknown for a mobile terminal. In this paper, we use a very simple but effective method to improve the system performance. First, the LS method is applied to Eqn. (3.7). It is given by

$$
\mathbf{h}_{\mathbf{f}_{-} \mathbf{s}}=\left(\operatorname{diag}\left(\mathbf{X}_{p}\right)\right)^{-\mathbf{1}} \mathbf{Y}_{p}
$$

where $\mathbf{h}_{\mathbf{f}_{-} \text {ls }}$ is LS vector result of frequency domain channel attenuations. Next, the following algorithm is performed:

1. Get the time domain CIR vector 


$$
\mathbf{h}_{\mathbf{t} \_\mathbf{s}}=i f f t\left(\mathbf{h}_{\mathbf{f} \_\mathbf{l s}}\right)
$$

2. Get $\mathbf{h}_{\mathbf{t}_{\_} \mathbf{e}}$ only with the most significant elements in $\mathbf{h}_{\mathbf{t} \_l s}$, say,

$$
\mathbf{h}_{\mathbf{t}_{-} \mathbf{e}}=\mathbf{h}_{\mathbf{t} \_ \text {ls }}, \quad \mathbf{h}_{\mathbf{t} \_\mathbf{e}}(L: N-1)=0
$$

where $L$ is the tap number of the channel.

3. Re-transform the time domain CIR $\mathbf{h}_{\mathbf{t} \_\mathbf{e}}$ to the frequency domain

$$
\mathbf{H}_{e}=\operatorname{diag}\left(f f t\left(\mathbf{h}_{\mathbf{t} \_} \mathbf{e}\right)\right)
$$

In fact, the information of tap number $L$ tells us the majority energy of the channel is located in the part of [0:L-1]. Eliminating the energy beyond this part will suppress the noise greatly while preserving the channel information well. In this step we will also estimate the noise variance in the frequency domain. After a simple calculation, it is given as

$$
\sigma_{w_{p}}^{2}=\operatorname{var}\left(\mathbf{h}_{\mathbf{t} \_\mathbf{l s}}(L: N-1)\right) g_{p}^{2} / N
$$

In the PT Mode, we need to transmit the pilot symbol for adequate periods of time to ensure the successful channel tracking in the DT Mode. After $S$ times' processing, we will get the following estimated results for the channel

$$
\left[\mathbf{H}_{e}(1), \mathbf{H}_{e}(2), \ldots, \mathbf{H}_{e}(S)\right]
$$

where the index signifies different transmitted symbol.

\section{B. DT Mode}

In DT Mode, there is no pilot. We should crack the channel estimation information from the received data blindly. This process is divided into two parts: one is channel tracking; the other is data detection and decision feedback channel estimation. 


\section{B.1. Channel Tracking}

The channel tracking problem can be depicted as follows: With $\mathbf{H}_{e}(1), \mathbf{H}_{e}(2), \ldots, \mathbf{H}_{e}(S)$ known, try to find $\hat{\mathbf{H}}_{e}(S+1)$. Without losing the generality, only one diagonal element $\hat{H}_{e . k}(S+1)$ is necessary to be considered, where $k \in[0, N-1]$ denotes frequency domain CSI index. Here the short range channel prediction [13] is employed to attain the current channel information based on the previous CSI observations. As mentioned in [27], the real part function $I_{e, k}(t)$ and imaginary part function $Q_{e, k}(t)$ of $H_{e, k}(t)$ can be regarded as the summations of many sinusoids, so their $S$ order derivatives are continuous. An Sdifferentiable function can be approximated by an $S-1$ order polynomial. The approximation error is determined by the following theorem [28]

Theorem 1: Given $a<b$, a function $f(x)$ with $S$ continuous derivatives on $[a, b]$, a polynomial $p(x)$ with degree $S$-1 so that

$$
p\left(x_{i}\right)=f\left(x_{i}\right)
$$

for $i=1, \ldots, S$, where the set $x_{i} \in[a, b]\left(x_{1}=\mathrm{a}\right.$ and $\left.x_{S}=\mathrm{b}\right)$ are distinct, then for every $x \in[a, b]$, there exists a point $\xi \in[a, b]$ such that

$$
f(x)-p(x)=\frac{\left(x-x_{1}\right) \ldots\left(x-x_{S}\right)}{S !} f^{(S)}(\xi)
$$

From Theorem 1, we find that the approximation error can be controlled with $\left\{x_{i}\right\}_{i=1}^{S}$ chosen properly. Thus, the approximation polynomials $\hat{I}_{e, k}(t)$ and $\hat{Q}_{e, k}(t)$ have the local information of $I_{e, k}(t)$ and $Q_{e . k}(t)$, respectively. $\hat{H}_{e . k}(S+1)$ can be easily found by extrapolating $\hat{I}_{e, k}(t)$ and $\hat{Q}_{e, k}(t)$ to the next sampling point. Next we describe the process 
of obtaining $\hat{I}_{e, k}(S+1)$, whereas $\hat{Q}_{e, k}(S+1)$ follows in the same way. Write $\hat{I}_{e, k}(t)$ as the polynomial form

$$
\hat{I}_{e, k}(t)=\sum_{i=0}^{S-1} c_{i} t^{i}
$$

satisfying $\hat{I}_{e, k}(t)=\left.I_{e, k}(t)\right|_{t=1 \ldots S}$. Clearly, $c_{i}$ can be found by solving the linear equations

$$
\mathbf{B c}=\mathbf{a}
$$

where $\mathbf{c}=\left[c_{0}, c_{1}, \ldots, c_{S-1}\right]^{T}, \mathbf{a}=\left[I_{e, k}(1), I_{e, k}(2), \ldots, I_{e, k}(S)\right]^{T}$ and

$$
\mathbf{B}=\left[\begin{array}{ccccc}
1 & 1 & 1 & \ldots & 1 \\
1 & 2 & 2^{2} & \ldots & 2^{S-1} \\
\vdots & \vdots & \vdots & \ldots & \vdots \\
1 & S-1 & (S-1)^{2} & \ldots & (S-1)^{S-1} \\
1 & S & S^{2} & \ldots & S^{S-1}
\end{array}\right]
$$

Therefore

$$
\mathbf{c}=\mathbf{B}^{-\mathbf{1}} \mathbf{a}
$$

Based on the result of Eqn. (3.14), $\hat{I}_{e, k}(S+1)$ will be obtained as

$$
\hat{I}_{e, k}(S+1)=\sum_{i=0}^{S-1} c_{i}(S+1)^{i}
$$

After obtaining $\hat{Q}_{e, k}(S+1)$ in the same way, $\hat{H}_{e, k}(S+1)$ has been attained. Now let us calculate the complexity of the channel prediction. $\mathbf{B}^{-\mathbf{1}}$ and $(S+1)^{i}$ should be calculated offline. Hence Eqn (3.17) needs $S^{2}$ multiplications and $S(S-1)$ additions and Eqn (3.18) needs $S-1$ additions and $S$ multiplications. As $\hat{h}_{e, k}(S+1)$ needs to be estimated for $k \in[0, N-1]$, the total time complexity is $o\left(S^{2} N\right)$. This method works very well in 
most cases. However, with the small SNR, the perturbation makes the prediction results far from the correct values. Hence, we use the following method to adjust the prediction values (This is for $\hat{I}_{e, k}(S+1)$ only, and $\hat{Q}_{e, k}(S+1)$ will follow in the same process)

1. Calculate the absolute difference between the consecutive points in $\left[I_{e, k}(1), I_{e, k}(2), \ldots, I_{e, k}(S)\right]$. Find the maximum $D_{\max }$ and the mean $D_{\text {mean }}$ of those absolute differences.

2. If $\operatorname{abs}\left(\hat{I}_{e, k}(S+1)-I_{e, k}(S)\right)>D_{\max }$

$$
\text { If } \begin{aligned}
& \left(\hat{I}_{e, k}(S+1)-I_{e, k}(S)\right)>0 \\
& \qquad \hat{I}_{e, k}(S+1)=I_{e, k}(S)+D_{\text {mean }}
\end{aligned}
$$

Else

$$
\hat{I}_{e, k}(S+1)=I_{e, k}(S)-D_{\max }
$$

End

End

After the adjustment, even for a small SNR, the prediction value has the acceptable precision.

\section{B.2. Data Detection and Decision Feedback Channel Estimation}

With the initial estimation of the channel $\hat{\mathbf{H}}_{e}(S+1)$, we begin to perform data detection and decision feedback channel estimation. The received data in the DT mode becomes

$$
\mathbf{Y}_{d}=\mathbf{X}_{d} \cdot \mathbf{H}_{d}+\mathbf{W}_{d}
$$

where $\mathbf{H}_{d}=\operatorname{diag}\left(H_{d, 0}, H_{d, 1}, \ldots, H_{d, N-1}\right)^{T}$ gives the frequency domain channel attenuations 
and $\mathbf{W}_{d}$ is the vector of the uncorrelated complex frequency domain Gaussian random noises. A novel PIC QMMSE/MMSE-PMRC Multi-User Detection/Channel Estimation method is exploited (PMRC denotes Partial MRC). This method first estimates the set of $b_{(m)}\left(m \in \Theta_{s}\right)$ (i.e., the modulated symbols of all the active users) by the combining method based on MMSE criterion. Then the data set is estimated again by MRC method after removing the multiple user interference components from the received signal. Finally the more precise channel estimation is obtained with decision feedback from the achieved data set. The process may be repeated several times to improve the performance. First, Let $\hat{\mathbf{H}}_{d}=\hat{\mathbf{H}}_{e}(S+1)$. The output of MMSE combiner for the user $k$ can be written as

$$
r_{M M S E(k)}=\mathbf{Q}_{M M S E(k)}^{H} \mathbf{Y}_{d} \quad 0 \leq k \leq N-1
$$

where $\mathbf{Q}_{M M S E(k)}=\left\{Q_{M M S E(k), 0}, \ldots, Q_{M M S E(k), N-1}\right\}^{T}$ stands for the MMSE equalization gain coefficients. Rooted in the MMSE criterion, the cost function $J_{(k)}$ is defined by

$$
J_{(k)}=E\left\{\left|e_{(k)}\right|^{2}\right\}=E\left\{\left|r_{M M S E(k)}-b_{(k)}\right|^{2}\right\} \quad 0 \leq k \leq N-1
$$

According to the well known Wiener-Hopf equation, the equalization gain vector which minimizes $J_{(k)}$ is defined as

$$
\mathbf{Q}_{M M S E(k)}=\mathbf{R}_{y y}^{-1} \mathbf{r}_{b y}
$$

where $\mathbf{R}_{y y}$ is the $N \times N$ auto correlation matrix and $\mathbf{r}_{b y}$ is the $N$ dimensional cross correlation vector. The $\mathbf{r}_{b y}$ is given by

$$
\begin{aligned}
\mathbf{r}_{b y} & =E\left\{\mathbf{Y}_{d} b_{(k)}^{(*)}\right\} \\
& =\hat{\mathbf{H}}_{d} \mathbf{G}_{(k)}
\end{aligned}
$$


Getting the auto correlation matrix $\mathbf{R}_{y y}$ is computationally excessive. Ignoring the impact of non-diagonal elements will lead to the following approximated results

$$
\mathbf{R}_{y y}=\operatorname{diag}\left(\left(\sigma_{d}^{2}+\frac{M}{N}\left|\hat{H}_{d, 0}\right|^{2}\right), \ldots,\left(\sigma_{d}^{2}+\frac{M}{N}\left|\hat{H}_{d, N-1}\right|^{2}\right)\right.
$$

where $\sigma_{d}^{2}$ is the frequency domain noise variance, and $\sigma_{w_{p}}^{2}$ from Eqn. (3.12) is used as its estimated value.

Therefore

$$
\mathbf{Q}_{M M S E(k)}=\left(\sigma_{d}^{2} \mathbf{I}+\frac{M}{N}\left|\hat{\mathbf{H}}_{d}\right|^{2}\right)^{-1} \hat{\mathbf{H}}_{d} \mathbf{G}_{(k)} \quad 0 \leq k \leq N-1
$$

Applying Eqn. (3.25) to (3.20), $r_{M M S E(k)}$ is obtained. Whereas we only know the number of active users, it is necessary to estimate the active users' data and the corresponding spread sequence mapping index set $\Theta_{s}$ from all the potential active users. Hence in Eqn. (3.25) and (3.20), the $k^{6} \mathrm{~s}$ area is from 0 to $N-1$. Now we sort all the $N$ elements of $\operatorname{abs}\left(r_{M M S E(k)}\right)$ in the decreasing order and get a vector $\mathbf{r}_{S O R T}$ and the corresponding mapping ordered index set $\Theta_{\text {sort }}$, which makes $\mathbf{r}_{\text {SORT }}=\mathbf{r}_{M M S E}\left(\Theta_{\text {sort }}\right)$. The result $\mathbf{r}_{S O R T}$ is divided into three parts. $\Phi_{A}$ is composed of more reliable data; $\Phi_{B}$ is the part including less reliable data and $0 s$, which needs further steps to handle; $\Phi_{C}$ is the part made of more reliable $0 s$. Obviously after sorting, the data nearby the index $M$ has less reliability. For this reason we obtain the part $\Phi_{A}, \Phi_{B}$, and $\Phi_{C}$ as follows:

1. Define a threshold $T_{s}, \quad T_{s}=[M / 5]+1$

2. $I_{\text {up }}=\min \left(M-1+T_{s}, N-1\right) \quad I_{\text {down }}=\max \left(M-1-T_{s}, 1\right)$

3. $\Phi_{A}=\mathbf{r}_{\text {SORT }}\left(0: I_{\text {down }}-1\right)$ 
Mapping index set: $\Theta_{A}=\Theta_{\text {sort }}\left(0: I_{\text {down }}-1\right)$

$$
\Phi_{B}=\mathbf{r}_{\text {SORT }}\left(I_{\text {down }}: I_{\text {up }}\right)
$$

Mapping index set: $\Theta_{B}=\Theta_{\text {sort }}\left(I_{\text {down }}: I_{\text {up }}\right)$

$$
\Phi_{C}=\mathbf{r}_{S O R T}\left(I_{u p}+1: N-1\right)
$$

The threshold is selected empirically. The principle of selection is to find a good balance between the complexity and performance. By applying hard decision to $\Phi_{A}$ we get the data vector result

$$
\mathbf{r}_{M M S E_{-} A}=\left[r_{M M S E_{-} A\left(i_{0}\right)}, \ldots, r_{M M S E_{-} A\left(i_{\left(I_{\text {down }}-1\right)}\right)}\right]^{T}
$$

where $\left\{i_{0}, \ldots, i_{\left(I_{\text {down }}-1\right)}\right\}=\Theta_{A}$. Afterward, the contribution of $I_{\text {down }}$ interfering users is subtracted from the received signal to get the corresponding user refined signal with reduced MAI.

$$
\tilde{\mathbf{Y}}_{\mathbf{M M S E}}=\mathbf{Y}_{\mathbf{d}}-\hat{\mathbf{H}}_{\mathbf{d}} \cdot \sum_{i \in \Theta_{A}} r_{M M S E_{-} A(i)} \mathbf{G}_{(i)}
$$

The remaining problem is to detect $M-I_{\text {down }}$ users' data in $\Phi_{B}$ with $\tilde{\mathbf{Y}}_{\text {MMSE }}$. Given that the data in $\Phi_{B}$ is less reliable, more powerful GMMSE method is utilized [29]. However, the complexity of solving a $N \times N$ linear system should be reduced for GMMSE method. First, we define a new vector,

$$
\mathbf{z}=\left[z_{0}, z_{1}, z_{2}, \ldots, z_{\left(I_{u p}-I_{d o w n}\right)}\right]^{T}=\mathbf{G}^{H} \hat{\mathbf{H}}_{d}^{*} \tilde{\mathbf{Y}}_{M M S E}
$$

where $\mathbf{G}=\left[\mathbf{G}_{\left(k_{0}\right)}, \mathbf{G}_{\left(k_{1}\right)}, \ldots, \mathbf{G}_{\left(k_{\left(I_{\text {up }}-I_{d o w n}\right)}\right)}\right]\left(\left\{k_{0}, k_{1}, \ldots, k_{\left(I_{\text {up }}-I_{\text {down }}\right)}\right\}=\Theta_{B}\right.$, the corresponding mapping index set of $\Phi_{B}$ ) and $\tilde{\mathbf{Y}}_{M M S E}=$ $\left[\tilde{Y}_{M M S E, 0}, \tilde{Y}_{M M S E, 1}, \ldots, \tilde{Y}_{M M S E, N-1}\right]^{T}$. The MMSE criterion applied to vector $\mathbf{z}$ and similarly to equation (3.21), we get the vector of decision variable as:

$$
\mathbf{r}_{G M M S E_{-} B}=\mathbf{Q}_{G M M S E}^{H} \mathbf{Z} \quad \text { with } \quad \mathbf{Q}_{G M M S E}=\mathbf{R}_{z z}^{-1} \mathbf{R}_{b z}
$$


For $\mathbf{R}_{z z}$ and $\mathbf{R}_{b z}$,

$$
\begin{aligned}
& \mathbf{R}_{z z}=E\left(\mathbf{z z}^{H}\right)=E\left(\mathbf{G}^{H} \hat{\mathbf{H}}_{d}^{*} \tilde{\mathbf{Y}}_{M M S E}\left(\mathbf{G}^{H} \hat{\mathbf{H}}_{d}^{*} \tilde{\mathbf{Y}}_{M M S E}\right)^{H}\right) \\
& =\mathbf{G}^{H} \hat{\mathbf{H}}_{d}^{*} E\left(\tilde{\mathbf{Y}}_{M M S E} \tilde{\mathbf{Y}}_{M M S E}^{H}\right) \hat{\mathbf{H}}_{d} \mathbf{G}
\end{aligned}
$$

It is easily known that

$$
E\left(\tilde{\mathbf{Y}}_{M M S E} \tilde{\mathbf{Y}}_{M M S E}^{H}\right)=\hat{\mathbf{H}}_{d} \mathbf{G G}{ }^{H} \hat{\mathbf{H}}_{d}^{H}+\sigma_{d}^{2} \mathbf{I}
$$

So

$$
\mathbf{R}_{z z}=\mathbf{G}^{H}\left|\hat{\mathbf{H}}_{d}\right|^{2} \mathbf{G G}^{H}\left|\hat{\mathbf{H}}_{d}\right|^{2} \mathbf{G}+\sigma_{d}^{2} \mathbf{G}^{H}\left|\hat{\mathbf{H}}_{d}\right|^{2} \mathbf{G}
$$

and

$$
\mathbf{R}_{b z}=E\{\mathbf{z b}\}=\mathbf{G}^{H}\left|\hat{\mathbf{H}}_{d}\right|^{2} \mathbf{G}
$$

where $\mathbf{b}=\left[b_{\left(k_{0}\right)}, b_{\left(k_{1}\right)}, \ldots, b_{\left(k_{\left(I_{\text {up }}-I_{\text {down }}\right)}\right)}\right]$.

Consequently,

$$
\mathbf{r}_{G M M S E_{-} B}=\left(\mathbf{G}^{H}\left|\hat{\mathbf{H}}_{d}\right|^{2} \mathbf{G}+\sigma_{d}^{2} \mathbf{I}\right)^{-1} \mathbf{G}^{H} \hat{\mathbf{H}}_{d}^{*} \tilde{\mathbf{Y}}_{M M S E}
$$

From the $\left(I_{u p}-I_{\text {down }}+1\right) \times 1$ result vector, $M-I_{\text {down }}$ results and corresponding mapping index set should be obtained. This can be simply done by selecting $M-I_{\text {down }}$ biggest elements in $\left|\mathbf{r}_{G M M S E_{-} B}\right|$. The result is acquired as

$$
\mathbf{r}_{G M M S E_{-} B_{-} R}=\left[r_{M M S E_{-} B\left(j_{0}\right)}, \ldots, r_{M M S E_{-} B\left(j_{\left(M-I_{d o w n}-1\right)}\right)}\right]^{T}
$$

where $\left\{j_{0}, \ldots, j_{\left(M-I_{d o w n}-1\right)}\right\}=\Theta_{B_{-} R}$, denoting the corresponding mapping index set of $\mathbf{r}_{\text {GMMSE_B }_{-} R}$. Combining the results of Eqn. (3.26) and (3.35), the final result and the corresponding mapping index set are equal to

$$
\begin{gathered}
\mathbf{r}_{M M S E_{-} R}=\left[\begin{array}{l}
\mathbf{r}_{M M S E_{-} A} \\
\mathbf{r}_{G M M S E_{-} B_{-} R}
\end{array}\right] \\
\Theta_{M M S E_{-} R}=\Theta_{A} \cup \Theta_{B_{-} R}
\end{gathered}
$$


It is clear that a $\left(I_{u p}-I_{\text {down }}+1\right) \times\left(I_{u p}-I_{\text {down }}+1\right)$ linear system needs to be solved instead of the original $N \times N$ linear system. With the trivial derivation, we get the upper limit for the $I_{u p}-I_{d o w n}+1$ as

$$
I_{\text {up }}-I_{\text {down }}+1<=2[M / 5]+3
$$

For most $M$, this upper limit $<[M / 2] \ll N$. Thus the system complexity is reduced significantly. In the system design, you can vary the tradeoff between the complexity and the performance by selecting different threshold value, which shows the good flexibility of the proposed system.

With $\mathbf{r}_{M M S E_{-} R}$, the system reconstructs the estimated interference of all users. Afterward, for every active user, the contribution of all the interfering users is subtracted from the received signal, and then MRC is used to get the data detection values. There are many reasons for the usage of the MRC in this stage:

1. The MAI has been cancelled by PIC. Even if there is new interference involved, it can be regarded as white noise. Hence MRC is quite effective in this case.

2. As mentioned in [30], the CE error variances for all taps are almost identical and small, thereby, making the total noise effectively white.

3. CQS can reduce the MAI of the system effectively with MRC.

A detailed explanation of the statement 3 is given next. First, let us apply MRC to Eqn. (3.19). Assuming that we know the channel estimation information and the $\Theta_{s}$ exactly, the MRC coefficients are given as

$$
\mathbf{Q}_{M R C(k)}=\left\{H_{d, 0} G_{(k), 0}, \ldots, H_{d, N-1} G_{(k), N-1}\right\}^{T}
$$

and then 


$$
r_{M R C(k)}=\mathbf{Q}_{M R C(k)}^{H} \mathbf{Y}_{d}
$$

The interference term for user $\mathrm{k}$ in Eqn. (3.40) can be written as

$$
I_{(k)}=\sum_{n=0}^{N-1}\left(\left|H_{d, n}\right|^{2}\left(\sum_{\substack{m \in \Theta_{s} \\ m \neq k}} b_{(m)} G_{(m), n}\right)+W_{d, n} H_{d, n}^{*}\right) G_{(k), n}^{*}
$$

Rewrite it as

$$
\begin{aligned}
I_{(k)} & =\sum_{\substack{m \in \Theta_{s} \\
m \neq k}} \sum_{n=0}^{N-1}\left|H_{d, n}\right|^{2} \frac{b_{(m)}}{N} e^{j \frac{2 \pi}{N} n(k-m)} & & \text { JMAI } \\
& +\sum_{n=0}^{N-1} H_{d, n}^{*} W_{d, n} e^{j \frac{2 \pi}{N} n k} G_{n}^{*} & & \text { JNoise }
\end{aligned}
$$

For MAI term only, applying the Plancherel theorem, we get

$$
I_{(k), M A I}=\sum_{\substack{m \in \Theta_{s} \\ m \neq k}} \sum_{n=0}^{N-1} h_{d, n}^{*} h_{d,(n-m+k)_{N}} b_{(m)}
$$

where $h_{d, n}$ is the time domain channel impulse response. Now it is assumed that $N>L$, i.e., $h_{n}=0$ when $n>L$. It can be found that when $( \pm(m-k))_{N}>L$ (The “" \pm " signifies both right and left directions, so it represents the cyclic shift distance between two users is larger than $L), \sum_{n=0}^{N-1} h_{d, n}^{*} h_{d,(n-m+k)_{N}}=0$. In other words, when the cyclic shift distance between two users is larger than the maximum delay spread of a multi-path fading channel $L$, there will be no MAI between them at all. Obviously in a full loaded MCCDMA downlink system, the maximum number of users which can cause interference is reduced from $N-1$ to $2 L$ for every user. For a low loaded system, if we arrange the cyclic shift distances among all the active users in a smart way (The distances are all larger than 
L). It is possible to eliminate MAI completely. Now consider Eqn. (3.19). If the difference between $H_{d}$ and $\mathrm{H}$ is small, those two can be regarded as approximately identical. Those interference users whose cyclic shift distance to the $k$ th user is larger than $L$ will have very small contributions to the MAI. Hence, the judgement errors of those users will not affect the result of MRC. That means a substantial improvement in the system performance.

Now, let us describe what we should do from the result of Eqn. (3.36) and (3.37) in a more mathematical way. First, for every active user, the contribution of all interfering users is subtracted to achieve better results

$$
\begin{gathered}
\tilde{\mathbf{Y}}_{\mathbf{M R C ( k )}}=\mathbf{Y}_{\mathbf{d}}-\hat{\mathbf{H}}_{\mathbf{d}} \sum_{i \in \Theta_{M R C(k)}} \mathbf{r}_{M M S E_{-} R(i)} \mathbf{G}_{(i)} \quad k \in \Theta_{M M S E_{-} R} \\
\Theta_{M R C(k)}=\Theta_{M M S E_{-} R} \cap \Theta_{\text {shift }(k)}
\end{gathered}
$$

where $\Theta_{\text {shift }(k)}$ denotes the set in which every number's cyclic shift distance to the $k$ th user is smaller than or equal to the maximum delay spread of a multi-path fading channel $L$ (Not including $k$ itself).

With the result of Eqn. (3.44), the MRC is applied.

$$
r_{M R C \_R(k)}=\mathbf{Q}_{\mathbf{M R C} \_\mathbf{R}(\mathbf{K})}^{\mathbf{H}} \tilde{\mathbf{Y}}_{\mathbf{M R C}(\mathbf{k})} \quad k \in \Theta_{M M S E_{-} R}
$$

where $\mathbf{Q}_{\mathbf{M R C} \_\mathbf{R}(\mathbf{K})}$ represents the MRC equalization gain coefficients, given as

$$
\mathbf{Q}_{\mathbf{M R C} \_\mathbf{R}(\mathbf{K})}=\left\{\hat{H}_{d, 0} G_{(k), 0}, \ldots, \hat{H}_{d, N-1} G_{(k), N-1}\right\}^{T}
$$

With the result of Eqn. (3.45), we can get

$$
\hat{\mathbf{X}}_{\mathbf{d}}=\sum_{m \in \Theta_{M M S E_{-} R}} \operatorname{HardDecision}\left(r_{M R C_{-} R(k)}\right) \mathbf{G}_{(\mathbf{m})}
$$


Following the same process in the PT mode, the decision feedback estimated CSI is obtained as $\hat{\mathbf{H}}_{\mathbf{d} \_\mathbf{r}}$. We assign $\hat{\mathbf{H}}_{\mathbf{d} \_\mathbf{r}}$ to $\hat{\mathbf{H}}_{\mathbf{d}}$ and redo the whole process from the Eqn. (3.20) until the estimated CSI converges or maximum iteration times have been achieved. Then the final results have been obtained. We store the current CSI estimation $\hat{\mathbf{H}}_{e}(u)=\hat{\mathbf{H}}_{\mathbf{d} \_\mathbf{r}}$, where $u$ is the current symbol index. The $\hat{\mathbf{H}}_{e}(u)$ is put into a first in first out (FIFO) queue and will be used as a previous CSI observation in the next channel prediction period. Finally, the data detection value of current mobile user $l$ can be obtained as

$$
b_{(l)}=\operatorname{HardDecision}\left(r_{M R C \_R(l)}\right)
$$

In summary, with the MMSE/QMMSE combination detection, the system puts more efforts into detecting more unreliable data, realizing a good tradeoff between the complexity and the performance. In the second interference cancellation part, due to the property of CQS, even for a full load system, only $2 L$ users need to be cancelled, which is much less than original $N-1$. This partial cancellation also improves the performance because the errors involved from the process of cancellation (Caused by imprecise channel estimation and erroneous user detections) have been greatly decreased

\subsubsection{Simulation and analysis}

The BER performance of the proposed system is evaluated by the use of software simulation. The main parameters used in the simulation are summarized in Table 3.1. 3GPP deployment channel models TR 25.943 are exploited in this work [31]. A typical urban channel model and a rural area channel model are tested. The maximum Doppler Frequency is set to $111 \mathrm{~Hz}$, which leads to a high speed mobile communication system. 


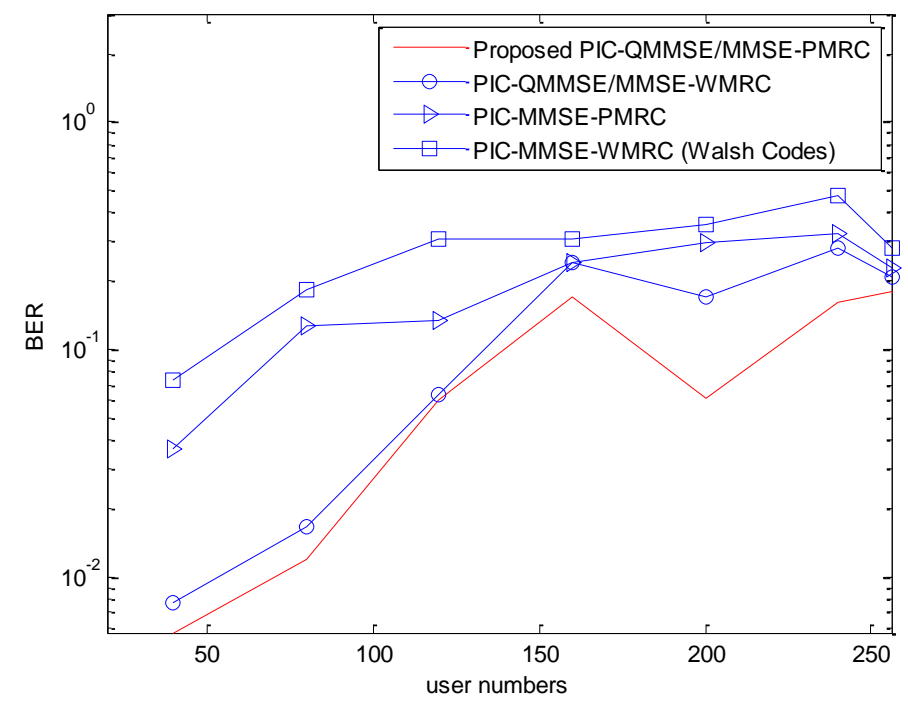

Figure 3.2. BER comparison of different receivers under 3GPP TR 25.943 typical urban channel model with $\mathrm{E}_{\mathrm{b}} / \mathrm{N}_{0}=0 \mathrm{~dB}$

During the simulation, 7 OFDM symbols of pilots are transmitted in PT mode period. After 96 DT OFDM symbols' transmission, the system enters another PT mode period. Figure 3.2 shows the BER simulation results under 3GPP TR 25.943 typical urban channel model with $\mathrm{E}_{\mathrm{b}} / \mathrm{N}_{0}=0 \mathrm{~dB}$. The solid curve is the proposed PIC-QMMSE/MMSEPMRC method. The solid with circles curve results from the proposed receiver with the complete cancellation strategy. On the contrary, the interference cancellation is partial in

Table 3.1: Main parameters used in simulations

\begin{tabular}{|c|c|c|c|}
\hline Signal bit rate & $12.5 \mathrm{MHz}$ & $\begin{array}{c}\text { Modulation } \\
\text { scheme }\end{array}$ & 8-PSK \\
\hline $\begin{array}{c}\text { Number of Data } \\
\text { sub-carriers } \\
\text { (FFT length) }\end{array}$ & 256 & Cyclic prefix & $5.21 \mathrm{us}$ \\
\hline $\begin{array}{c}\text { Maximum Doppler } \\
\text { Frequency }\end{array}$ & $111 \mathrm{~Hz}$ & Channel model & $\begin{array}{c}\text { 3GPP TR 25.943 urban } \\
\text { and rural channel model }\end{array}$ \\
\hline $\begin{array}{c}\text { Spreading } \\
\text { Sequences }\end{array}$ & $\begin{array}{c}\text { Walsh } \\
\text { codes }\end{array}$ & $\begin{array}{c}\text { OFDM symbol } \\
\text { period }\end{array}$ & 20.84 us \\
\hline $\begin{array}{c}\text { PT OFDM symbol } \\
\text { number in one } \\
\text { period }\end{array}$ & 7 & $\begin{array}{c}\text { DT OFDM } \\
\text { symbol number } \\
\text { in one period }\end{array}$ \\
\hline
\end{tabular}




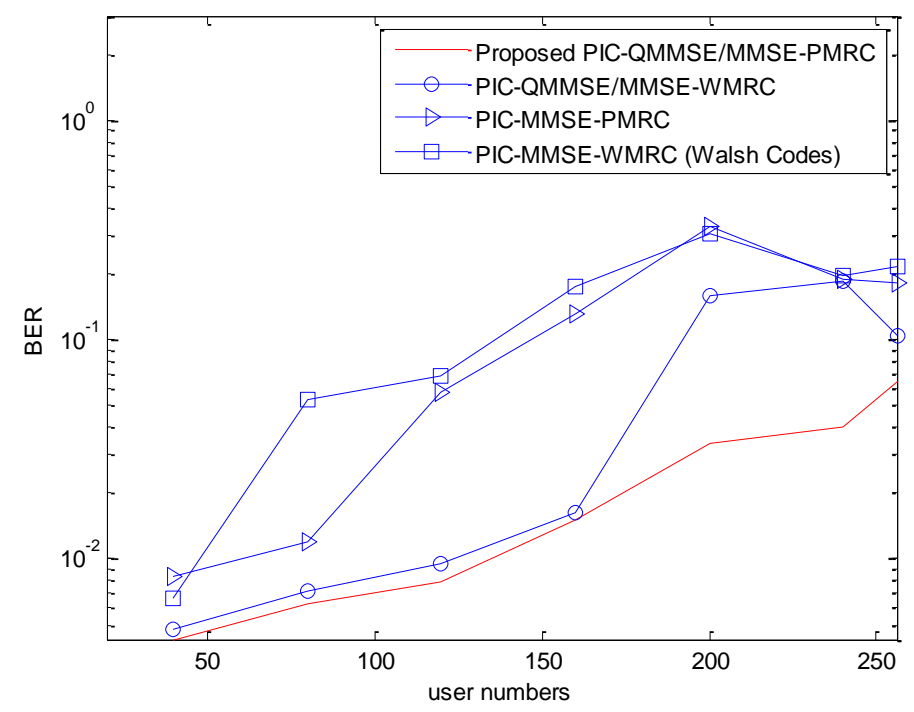

Figure 3.3. BER comparison of different receivers under 3GPP TR 25.943 typical rural channel model with $\mathrm{E}_{\mathrm{b}} / \mathrm{N}_{0}=0 \mathrm{~dB}$

the first method. We named it PIC-QMMSE/MMSE-WMRC where WMRC denotes the complete MRC. The solid curve with triangles represents PIC-MMSE-PMRC, i.e., the pure MMSE combining is employed instead of the combination of QMMSE and MMSE. Finally, besides all above three receivers with CQS, the result of traditional PIC-MMSEWMRC (Walsh Codes) receiver is marked as the solid curve with squares. The simulation results under 3GPP TR 25.943 typical rural channel model are given in Figure 3.3 in the same way.

In Figure 3.2 and 3.3, both methods with QMMSE/MMSE outperform those two with pure MMSE. Because the precision of initial detection is very important in the interference cancellation based MUD, it is quite worthwhile to refine unreliable data with the proposed QMMSE/MMSE method. It brings a drastic improvement with a moderate increase in complexity. We also find that the PIC-MMSE-PMRC (CQS) outperforms the PIC-MMSE-WMRC (Walsh Codes) in both figures. This is due to the unique MAI 
suppression advantage of CQS in the multi-user environment as shown in [12]. CQS can reduce the PAPR effectively as well because of its constant envelope property. Since PAPR and MAI minimization are two very important issues, it is reasonable to believe that the CQS is a good choice for MC-CDMA systems. After comparing the results of the proposed PIC-QMMSE/MMSE-PMRC and PIC-QMMSE/MMSE-WMRC, we find that the proposed method only brings minor advantage in a low loaded system but that advantage becomes more explicit when the number of active users increases. In a low loaded system, the system is prone to getting more precise channel estimation information and user detection values. There are no significant differences if we cancel part of them or all of them. However when the number of active users is increased, the imprecise channel estimation and erroneous user detection become more and more serious. Consequently, more errors have been involved in the process of the data cancellation. Hence only canceling part of them not only decreases the complexity but also mitigates the errors brought by the interference cancellation process.

The channel tracking minimum square errors (MSE) results for a full-loaded MC-CDMA system are shown in Figure 3.4 and 3.5 that $\mathrm{E}_{\mathrm{b}} / \mathrm{N}_{0}$ is set to $5 \mathrm{~dB}$ and $20 \mathrm{~dB}$, respectively. In Figure 3.4, the proposed method has the smallest channel MSE although all methods are losing their channel track gradually as time goes by. In addition, the channel MSE tracking performance and BER order of all methods corresponds to BER diagram in Figure 3.2 and Figure 3.3 completely. So the similar analysis can be applied. Furthermore, it deserves to be noticed that the PIC-MMSE-PMRC method has a better performance than that of PIC-QMMSE/MMSE-WMRC (CQS) in both channel tracking and BER under $20 \mathrm{~dB}$ condition. 


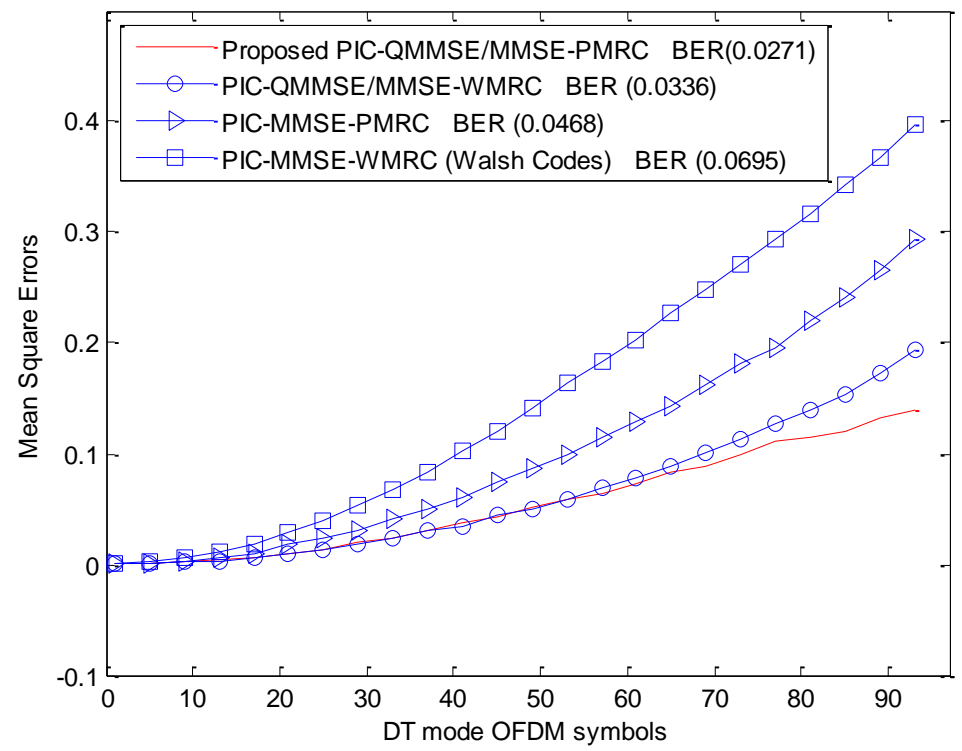

a) Under 3GPP TR 25.943 typical urban channel model

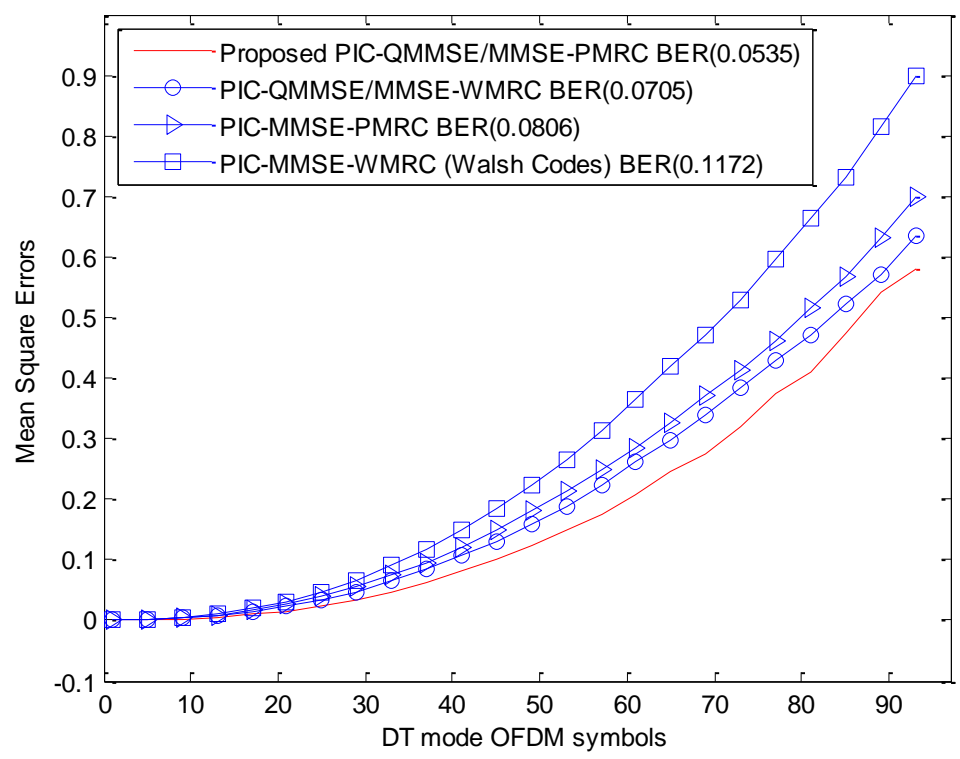

b) Under 3GPP TR 25.943 typical rural channel model

Figure 3.4. Channel MSE comparison of full loaded receivers with $\mathrm{E}_{\mathrm{b}} / \mathrm{N}_{0}=5 \mathrm{~dB}$ 


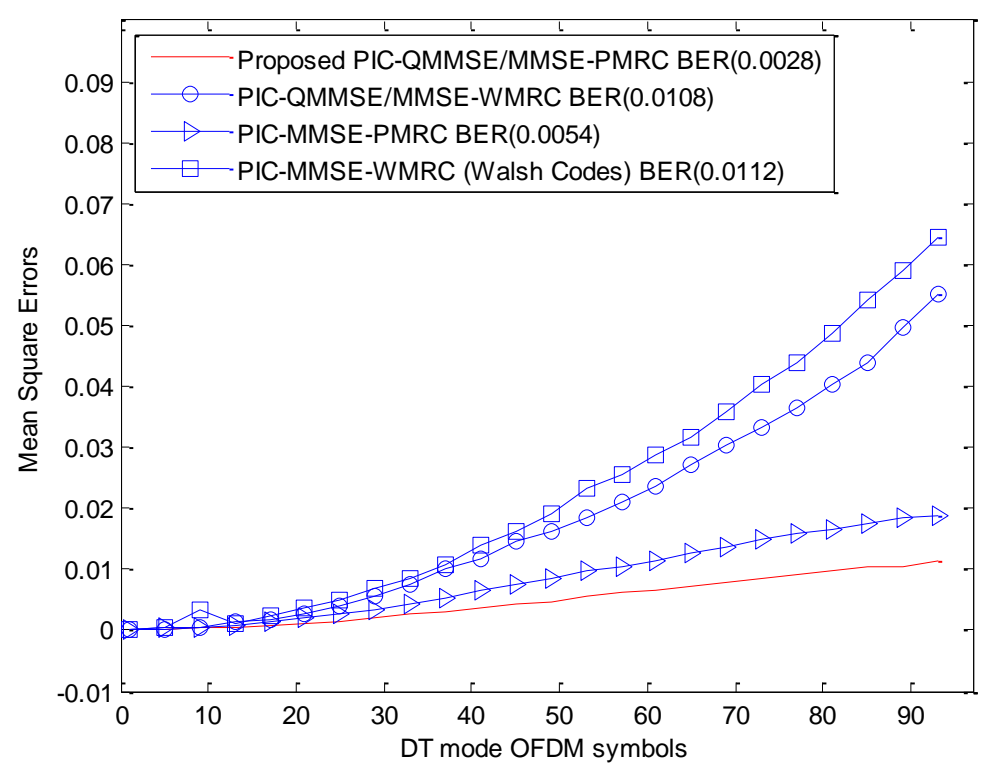

a) Under 3GPP TR 25.943 typical urban channel model

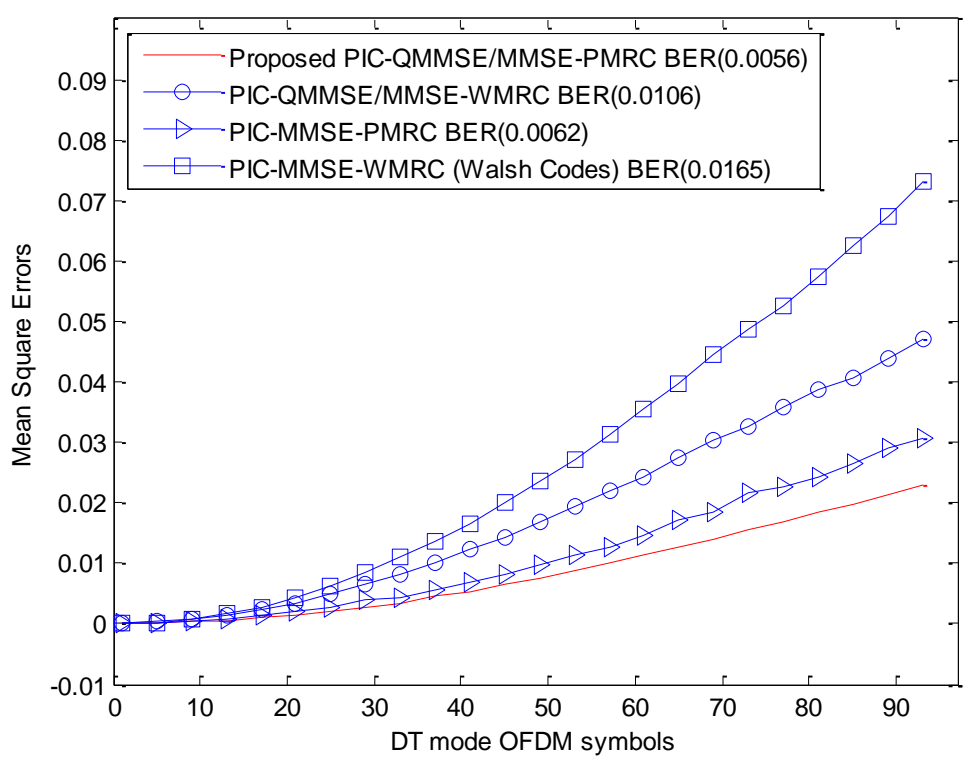

b) Under 3GPP TR 25.943 typical rural channel model

Figure 3.5. Channel MSE comparison of full loaded receivers with $E_{b} / N_{0}=20 d B$ 


\subsection{Receiver for Uplink MC-CDMA Systems}

Recently, in uplink MC-CDMA systems, pre-equalization at the transmitter have been proposed [32] [33]. In [34] different single-user pre-equalization techniques for uplink MC-CDMA systems have been compared and the corresponding power limiting issue has been discussed as well. As pointed in [35], the pre-equalization method is based on the reciprocity of the uplink and downlink channel responses for the time division duplex (TDD) in the same frequency bandwidth. This allows that each fixed user can preequalize uplink transmission signal in the stationary multi-path fading channel by using the channel response estimated during the downlink transmission. In the time-varying channels with mobile users, there is however a quite big difference between the channel responses during the uplink and downlink transmission time. From this fact, if the channel response estimated directly from the downlink is used for the pre-equalization at the user terminals, it is impossible to keep the orthogonality in the received multiplexed uplink signal at the base station.

\subsubsection{System Description}

The proposed MC-CDMA uplink transmitter is shown in Figure 3.6. Instead of using the channel response estimated during the downlink transmission directly, we exploit the techniques in Section 3.2.1 to get $\mathbf{H}_{n}=\operatorname{diag}\left(H_{n, 0}, H_{n, 1}, H_{n, 2}, \ldots, H_{n, N-1}\right)$, representing the channel prediction of next sampling point. After the CQS spreading, the data on every sub-carrier should be pre-equalized with the pre-equalization coefficients

$$
\mathrm{E}_{p}=\left(E_{p, 0}, E_{p, 1}, E_{p, 2}, \ldots, E_{p, N-1}\right)
$$




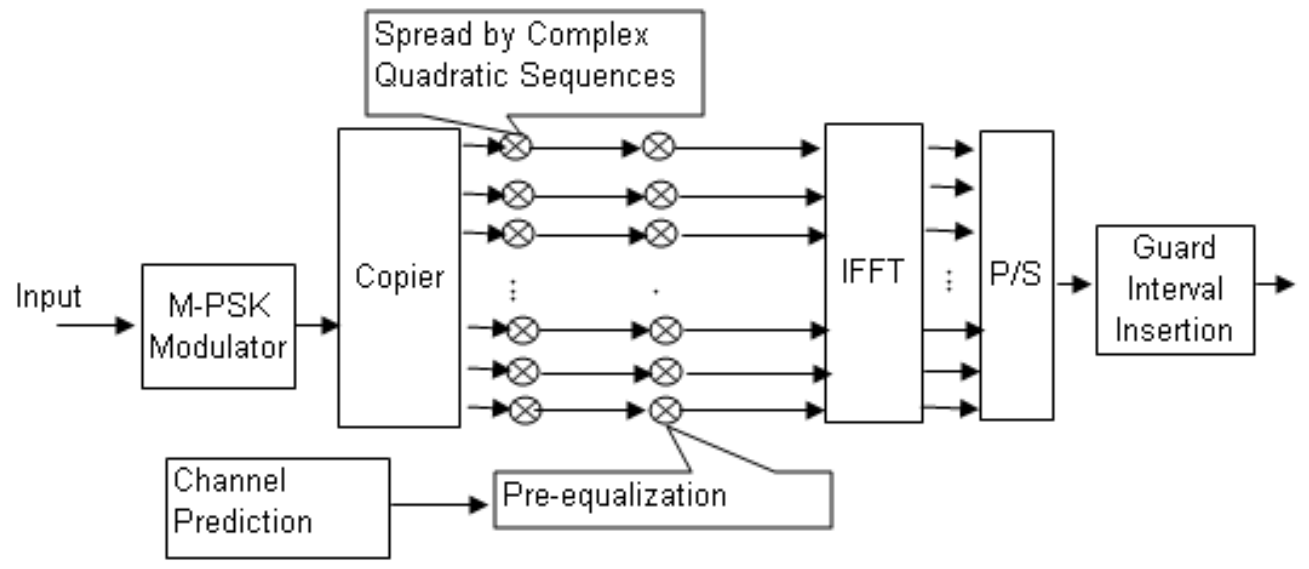

Figure 3.6. MC-CDMA uplink transmitter

The pre-equalization coefficients will be determined by the following three steps.

1. Pre-Orthogonality Restoring Combining (PRE-ORC) will be used in the first step.

$$
E_{p-1, k}=\frac{1}{H_{n, k}} \quad 0 \leq k \leq N-1
$$

2. For some sub-carriers with the deep channel fading, the pre-equalization will make PAPR extremely high. Therefore we perform a nonlinearity clipping

$$
E_{p-2, k}= \begin{cases}E_{p-1, k}, \quad \text { if }\left|E_{p-1, k}\right|<A_{0} & \\ A_{0} e^{j \arg \left(E_{p-1, k}\right)} & 0 \leq k \leq N-1\end{cases}
$$

where $A_{0}$ is PAPR limiting threshold, which depends on your PAPR reducing requirement.

3. To satisfy the power limiting for pre-equalization, a normalization factor is employed in conjunction with pre-equalization

$$
E_{\text {norm }}=\frac{1}{\sqrt{\frac{1}{P_{c}} \sum_{k=0}^{N-1}\left|E_{p-2, k}\right|}}
$$


where $P_{c}$ is the constraint on the total transmit power per OFDM symbol.

$$
E_{p, k}=E_{\text {norm }} E_{p-2, k} \quad 0 \leq k \leq N-1
$$

Due to the constant envelope property of CQS, the transmitted signal's PAPR is completely determined by the channel frequency response. In general, we can assume that the amplitude distribution in both the in-phase (I) and the quadrature phase (Q) follows a complex Gaussian, or in other words, a Rayleigh Distribution with the same standard deviation.

This probability density function (PDF) of the channel amplitude is given in Figure 3.7. This figure is generated from the typical urban channel model and a vehicle velocity of 80 miles/hour. According to Figure 3.7, the probability of the signal amplitude exceeding the $8 \mathrm{~dB}$ mark is about 0.0028 . Therefore, the clipping probability per subcarrier for the proposed MC-CDMA system is 0.0028 if the amplifier back-off value is $8 \mathrm{~dB}$. This probability is small enough so we will ignore its effect in the following analysis.

On the receiver side, the $\mathrm{BS}$ receives the signals from the different mobile terminals. The proposed MC-CDMA system is quasi-synchronous in uplink side, i.e. the timing of all users is aligned within in a small synchronization window (Within the margin of the cyclic prefix). This assumption is suitable for the reversed link in practice [11]. Power control is required for a realistic CDMA system because of what is known as the near-far problem [36]: users far from the base station experience far greater path loss than users that are near the base station. Optimum power control can be achieved when all users are decoded with the same signal-to-interference ratio (SIR). Otherwise a user with a low SIR dominates the BER performance of the system, which is defined as the average BER over all users. In this work, we adopt the commercial CDAM system power control 


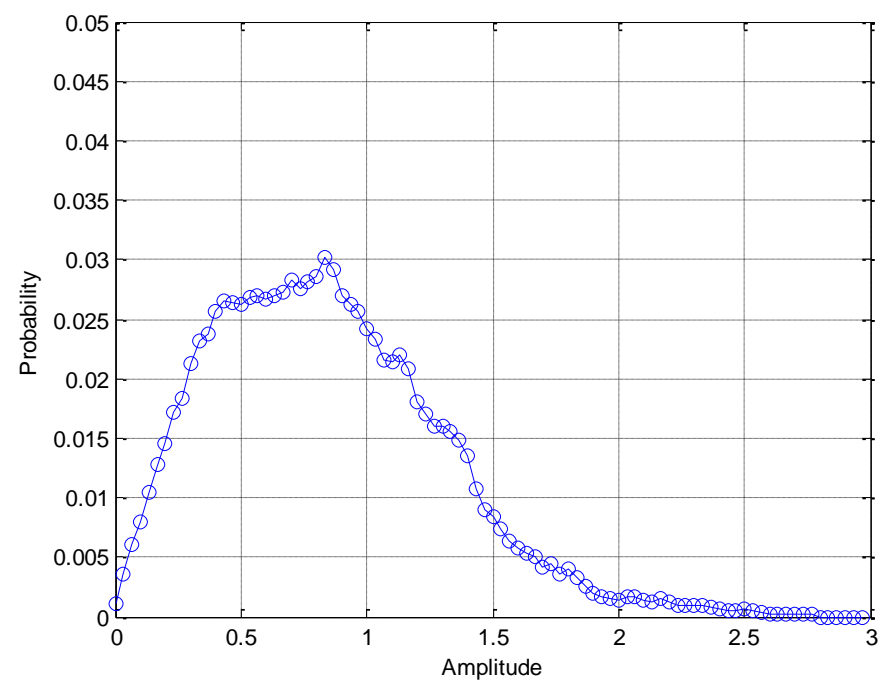

Figure 3.7. Channel amplitude PDF

scheme. That is, with a tight feedback loop, all the user's signals arrive at the base station with approximately the same power, which results in a consistent quality of service (QoS).

In uplink MC-CDMA systems, there are a lot of work focuses on the interference cancellation based multiple user detection. Some of them involve the channel decoding in the multiple user detection iteration process. However, we believe this will bring the problems in terms of the complexity, latency, and error amplification caused by the decoder in every iteration step. The partial parallel interference cancellations (PPIC) with its adaptive implementation are investigated for MC-CDMA system by Mozaffaripour et al. [37]. Kaiser and Hagenauer proposed a novel soft-parallel IC (SPIC) for MC-CDMA systems that exchanges soft output information between the IC receiver and the channel decoder iteratively [38]. In 2004, Benvenuto et al. extended Kaiser's work [38] to multi-level quadrature amplitude modulation (QAM) constellations [39]. However, to the best of our knowledge, SPIC receivers have never been considered for 
turbo coded MC-CDMA systems. Although research of iterative PIC has been done for CDMA systems [40-42], the corresponding work for MC-CDMA systems is still inadequate. Basically, how to apply iterative PIC techniques into an uplink MC-CDMA system in order to gain robust performance with low complexity is always a challenging task for next-generation communication systems.

This work proposes a novel iterative partial SPIC receiver for turbo coded uplink MCCDMA systems. We extend the work in [39] to multi-stage SPIC, and combine it with the iterative turbo decoder. In addition, the new adaptive minimum mean square combining (MMSEC) is applied. The MC-CDMA system is therefore capable of adjusting the equalization gain coefficients in every iterative stage adaptively. Unlike in the work described in [40-42], we avoid the problems in terms of the complexity, latency, and error amplification caused by the decoder in every iteration step. Instead, the soft in soft out (SISO) inverse bit mapper is employed and its output is fed back to the SISO bit mapper directly. In the last stage, when the majority of the interference is cancelled out, the output of SISO inverse bit mapper will go through the turbo decoder and decision device to build the final output. Both analysis and simulation indicate that our proposed iterative partial SPIC receiver achieves a considerably better performance with a low complexity structure compared with traditional systems.

In this part, we will use Walsh codes, quadrature amplitude modulation (QAM) constellations and Turbo codes to describe the whole system. However, the LDPC and other constellations also work well and the CQS can be employed instead of Walsh codes to improve the system performance further. 


\subsubsection{Iterative Partial SPIC receiver for turbo coded uplink MC- CDMA systems}

Iterative partial SPIC receiver for turbo coded uplink MC-CDMA systems is described in detail in this section. On the receiver side (base station) in Figure 3.8, following the removal of the Guard Interval, the received signal is transferred from a serial stream into $N$ parallel streams. Then the data is processed by the FFT unit. After that, the received data of the $k$-th sub-carrier becomes

$$
Y_{k}=\sum_{m=0}^{M-1} b_{(m)} G_{(m), k} H_{(m), k}+W_{k}
$$

where $b_{(m)}$ is the current message symbol of user $m$ after processing of the modulator, $\left\{G_{(m), 0}, G_{(m), 1}, G_{(m), 2}, \ldots, G_{(m), N-1}\right\}$ are the $m$-th user's frequency spreading sequences, $H_{(m), k}$ gives the frequency domain channel attenuation on the $k$-th sub-carrier of the $m$ th user, $W_{k}$ is the uncorrelated complex frequency domain Gaussian random noise with the variance $\sigma^{2}$. In Figure 3.8 the SPIC Receiver has been divided into three parts: PIC component, the forward path and the backward path for every user. In one stage of multistage operation, the PIC component collects the estimated interference of all users from the backward paths in the last stage. Afterward, for every active user, the contribution of all interfering users is subtracted from the received signal to get the corresponding user refined signal with reduced MAI. This refined signal can be used in the forward path to generate more reliable detection result output. Also, it will be fed back to PIC component through the backward path, generating more reliable interference information, which will 


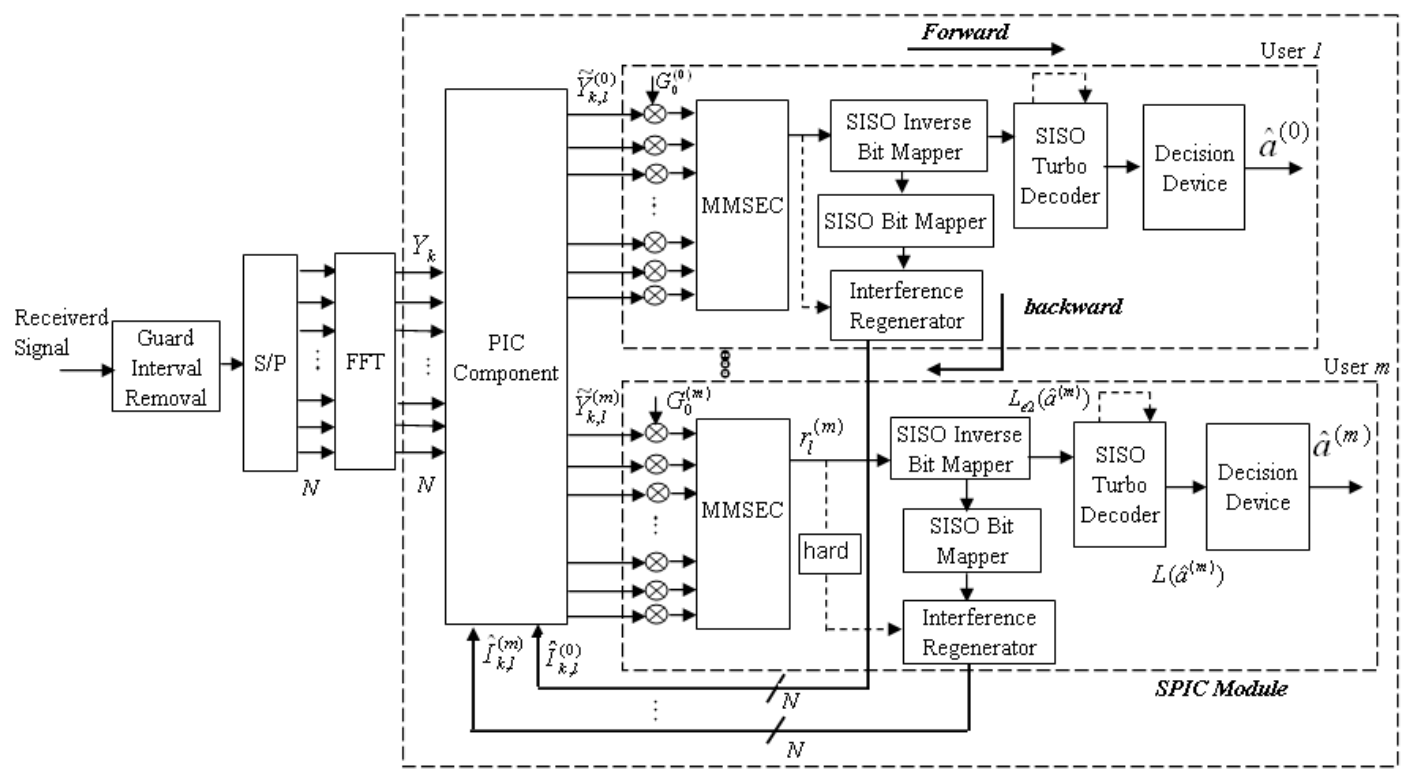

Figure 3.8. The proposed SPIC Receiver

make a positive impact on the next iteration. After several iterations (stages), the result will become more and more reliable. More details are described in the following subsections.

\section{A. PIC Component}

The PIC component has two inputs and one output. One input is $Y_{k}$ from Eq. (3.54). The other one is the estimated interference from the backward paths of all users, denoted as $\hat{I}_{(m), k, l}$, where $m, k$ and $l$ represent the user number, the subcarrier number and the iteration number, respectively. The output is the signal for each user after interference cancellation, written as $\tilde{Y}_{(m), k, l}$, where $m, k$ and $l$ have the same meaning with $\hat{I}_{(m), k, l}$. The PIC component is used to partially cancel the interference at each stage and the algorithm can be expressed as: 


\section{Initialization:}

Stage number $l \leftarrow 1$

For all $m$ and $k \quad \tilde{Y}_{(m), k, l-1} \leftarrow Y_{k} \quad$ End

Pass $\tilde{Y}_{(m), k, l-1}$ (for all $m$ and $k$ ) to forward path, and get

$\hat{I}_{(m), k, l}($ for all $m$ and $k$ ) from the backward path.

Recursion:

For all $m$ and $k$

$$
\tilde{Y}_{(m), k, l}=p_{l}\left(Y_{k}-\sum_{i=0, i \neq m}^{M-1} \hat{I}_{(i), k, l}\right)+\left(1-p_{l}\right) \tilde{Y}_{(m), k, l-1}
$$

End

Pass $\tilde{Y}_{(m), k, l}$ (for all $m$ and $k$ ) to forward path, and get

$\hat{I}_{(i), k, l+1}($ for all $i$ and $k$ ) from the backward path.

$l \leftarrow l+1$

In the algorithm $p_{l}$ denotes the partial cancellation weight at stage 1 . The use of weight $p_{l}$ is aimed to reduce the cost of wrong MAI estimations. The weight will be increased in the iteration process as the estimation becomes more reliable in later stages. To reduce the computation cost, the weight are pre-assigned rather than computed exactly as in [43].

\section{B. Forward path}

Since the same structures for all the users work in parallel for forward and backward path, only the $m$ th user processing flow is depicted. For the spreading and MMSE combiner, the output of MMSE combiner can be written as 


$$
r_{(m), l}=\sum_{k=0}^{N-1} \tilde{Y}_{(m), k, l} G_{(m), k}^{*} Q_{(m), k, l}^{*}
$$

where $r_{(m), l}$ denotes the result of the $m$ th user's $l$ iteration and $Q_{(m), k, l}$ stands for the MMSE equalization gain coefficients $\left(m, k\right.$ and $l$ have the same meaning with $\tilde{Y}_{(m), k, l}$ ). The problem is to find the appropriate $Q_{(m), k, l}$. Considering the initial case of $l$ equal to 0 first, Eqn. (3.55) becomes

$$
r_{(m), 0}=\sum_{k=0}^{N-1} \tilde{Y}_{(m), k, 0} G_{(m), k}^{*} Q_{(m), k, 0}^{*}=\sum_{k=0}^{N-1} Y_{k} G_{(m), k}^{*} Q_{(m), k, 0}^{*}
$$

Based on the MMSE criterion, the cost function $J_{(m), 0}$ is defined by

$$
J_{(m), 0}=E\left\{\left|e_{(m), 0}\right|^{2}\right\}=E\left\{\left|r_{(m), 0}-b_{(m)}\right|^{2}\right\}
$$

According to the well known Wiener-Hopf equation, the equalization gain vector for minimizing $J_{(m), 0}$ is defined as

$$
\vec{Q}_{(m), 0}=R_{y y}^{-1} \vec{r}_{b y}
$$

where $\left(\vec{Q}_{(m), 0}\right)^{H}=\left(Q_{(m), 0,0}^{*}, Q_{(m), 1,0}^{*}, Q_{(m), 2,0}^{*}, \ldots, Q_{(m), N-1,0}^{*}\right)$ and $R_{y y}$ is the auto correlation matrix of $N \times N$ dimension and $\vec{r}_{b y}$ is the $N$ dimensional cross correlation vector. The $k$ th element of $\vec{r}_{b y}$ is given by

$$
\begin{aligned}
r_{b y, k} & =E\left\{Y_{k} G_{(m), k}^{*} b_{(m)}^{*}\right\} \\
& =\frac{H_{(m), k}}{N} C_{Q A M}
\end{aligned}
$$

where $C_{Q A M}$ is a constant related to QAM, which equals to $\frac{d}{3}\left(L_{q}-1\right)\left(L_{q}\right.$ is the constellation points number of QAM, and $d$ is the half distance between two nearest neighbors in the constellations). Getting the auto correlation matrix $R_{y y}$ is 
computationally excessive. By ignoring the impact of off-diagonal elements, it will lead to the following approximated results

$$
\begin{aligned}
& R_{y y}=\operatorname{diag}\left(\frac{1}{N}\left(\sigma^{2}+\frac{1}{N} C_{Q A M} \sum_{m_{0}=0}^{M-1}\left|H_{\left(m_{0}\right), 0}\right|^{2}\right),\right. \\
& \left.\ldots, \frac{1}{N}\left(\sigma^{2}+\frac{1}{N} C_{Q A M} \sum_{m_{0}=0}^{M-1}\left|H_{\left(m_{0}\right), N-1}\right|^{2}\right)\right)
\end{aligned}
$$

Hence

$$
\begin{array}{r}
Q_{(m), k, 0}=\rho \frac{H_{(m), k} C_{Q A M}}{\sigma^{2}+C_{Q A M} \frac{1}{N} \sum_{m_{0}=0}^{M-1}\left|H_{\left(m_{0}\right), k}\right|^{2}} \\
\rho=\frac{1}{\sum_{k=0}^{N-1} \frac{\left|H_{(m), k}\right|^{2} C_{Q A M}}{N\left(\sigma^{2}+C_{Q A M} \frac{1}{N} \sum_{m_{0}=0}^{M-1}\left|H_{\left(m_{0}\right), k}\right|^{2}\right)}}
\end{array}
$$

where $\rho$ is the normalization factor in order to cope with high order modulations. For later iterations, the MMSE equalization gain coefficients equal to

$$
Q_{(m), k, l}=\rho \frac{H_{(m), k} C_{Q A M}}{\sigma^{2}+C_{Q A M} \frac{1}{N}\left(\left(1-p_{l}\right)^{2} \sum_{\substack{m_{0}=0 \\ m_{0} \neq m}}^{M-1}\left|H_{\left(m_{0}\right), k}\right|^{2}+\left|H_{(m), k}\right|^{2}\right)} \quad l \geq 1
$$

and

$$
\rho=\frac{1}{\sum_{k=0}^{N-1} \frac{\left|H_{(m), k}\right|^{2} C_{Q A M}}{N\left(\sigma^{2}+C_{Q A M} \frac{1}{N}\left(\left(1-p_{l}\right)^{2} \sum_{\substack{m_{0}=0 \\ m_{0} \neq m}}^{M-1}\left|H_{\left(m_{0}\right), k}\right|^{2}+\left|H_{(m), k}\right|^{2}\right)\right)}}
$$

where $p_{l}$ indicates the partial cancellation weight at stage $l$. Since the weight $p_{l}$ will be increased in the iteration process, the system will be able to adjust the equalization gain coefficients adaptively with different interference estimation result in every stage. The soft in soft out (SISO) inverse bit mapper, which is same with the one in [39] completely 
appears behind the MMSEC. After that, the output of the SISO inverse bit mapper will be fed back to the SISO Bit Mapper directly. In the last stage (i.e., when the majority of interference has been cancelled out), the output of the SISO inverse bit mapper will go through the turbo decoder and the decision device to build the final output. The turbo decoder is a typical feedback decoder as shown in [44] with its logarithmic maximum a posteriori (Log-MAP) version. The a posteriori log-likelihood ratio (LLR) output $L\left(\hat{a}_{(m)}\right)$ can be used to generate the hard-decision output by the decision device.

\section{Backward path}

The basic idea of a SISO bit mapper in the work of Benvenuto et al. [39] is applied to transfer the LLR output to the symbol domain. In [39], the pure SISO algorithm is time consuming. Therefore, we employ a simplified method. The output of the SISO bit mapper [39] is evaluated as

$$
\hat{b}_{(m), l}=E\left(b_{(m), l}\right)=\sum_{\alpha \in A} \alpha P\left[b_{(m), l}=\alpha\right]
$$

where $P\left[b_{(m), l}=\alpha\right]$ can be obtained from the $L L R$ values generated by SISO inverse bit mapper, and $A$ is the whole QAM constellation space. However in fact, only those points that are adjacent to the output value of MMSEC in the constellation space have dominant effects on the result of the SISO bit mapper. Hence we modify the set from $A$ to $A$ ', which only includes four nearest elements in the whole QAM constellation space. In Figure 3.8, the interference regenerator block of user $\mathrm{m}$ reconstructs the interference with the following term

$$
\hat{I}_{(m), k, l}=\hat{b}_{(m), l} G_{(m), k} H_{(m), k}
$$




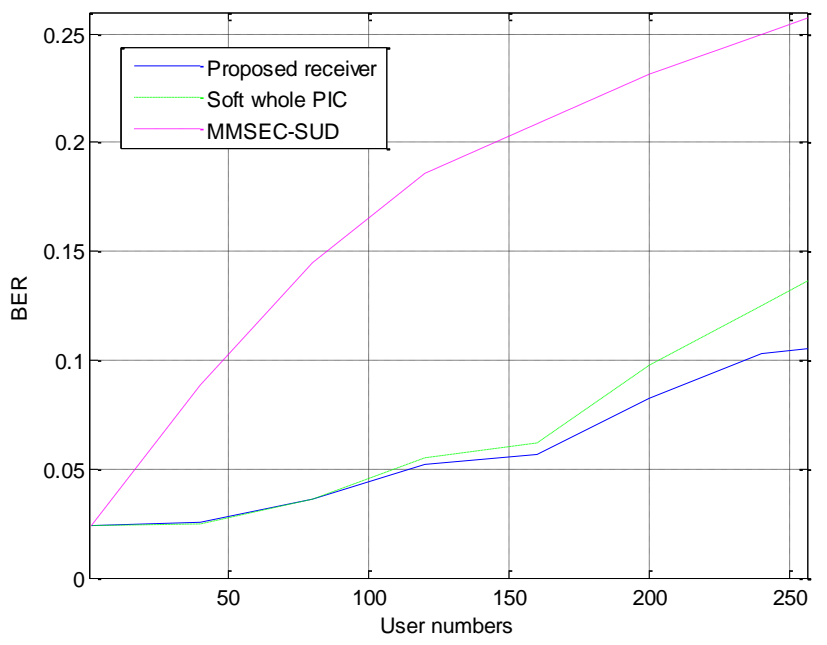

Figure 3.9. BER comparison of different receivers under channel SUI-2

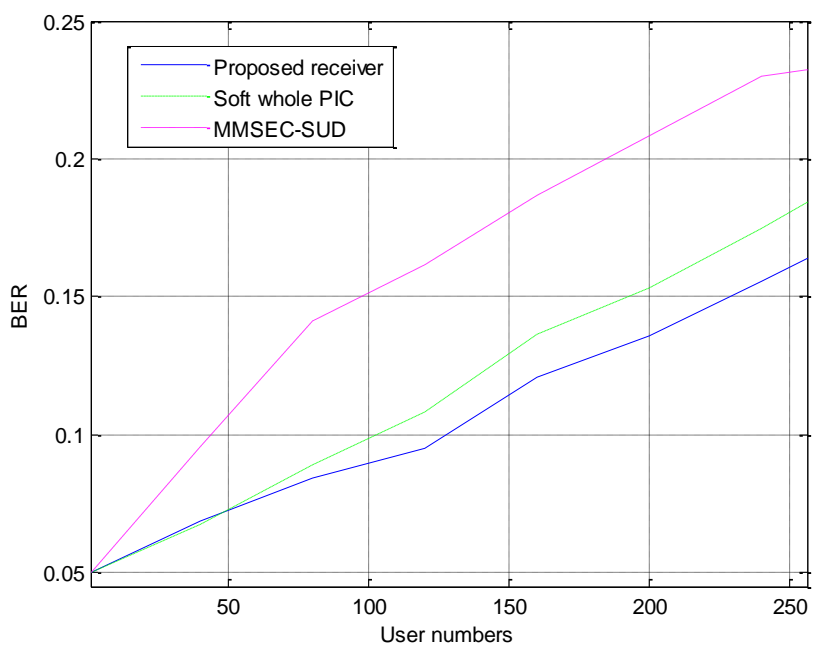

Figure 3.10. BER comparison of different receivers under channel SUI-6 


\subsubsection{Simulation results and analysis}

The BER performance of the proposed iterative partial SPIC receiver for turbo coded uplink MC-CDMA systems is evaluated by the use of software simulation. The main parameters used in the simulation are summarized in Table 3.2.

There are six omni-directional antenna channel models which appear in [14]. From all of them, we select two as our test channels: SUI-2, and SUI-6. SUI-2 has low delay spread and SUI-6 has high delay spread. The results of MMSEC SUD turbo decoder receiver are presented as a reference. In our proposed receiver, 4-stage cancellation is applied and the partial cancellation weight array is equal to $\left(p_{1}=0.6, p_{2}=0.7, p_{3}=0.8, p_{4}=0.9\right)$. The result of proposed receiver with whole cancellation strategy is given to show the effect of partial cancellation. From all the subcarriers, only some of them are selected as observation points to represent the whole channel attenuation array. This can be used to simulate channel estimation errors brought by the comb-type pilot effectively.

Figure 3.9 shows the simulation results under channel SUI-2. The solid curve corresponds to our proposed method. The second curve (short dashed) results from proposed receiver with whole cancellation strategy (whole soft PIC receiver). Finally, the result of MMSEC SUD turbo decoder receiver is represented as long dashed curve. The simulation results under channel SUI-6 are given in Figure 3.10 in the same way. The maximum Doppler Frequency is set to $120 \mathrm{~Hz}$, which leads to a high speed mobile communication system. In this case, the channel estimation errors are quite serious. Hence even a single user suffers strong BER degradation, and the situation gets worse for multiple users. In both Figure 3.9 and 3.10, the results of SUD receiver are greatly 
improved by IC receiver, which indicates the huge potential of interference cancellation methods. It shows that the proposed receiver gives the best performance either the channels are high (SUI-6) or low delay spread (SUI-2). The results show that the partial IC has obvious advantage over whole IC. The phenomenon becomes apparent when there are a large number of active users in the system.

\subsubsection{Conclusion}

A novel multi-stage partial soft parallel interference cancellation receiver for QAM modulated turbo coded uplink MC-CDMA systems is proposed. Since the new adaptive minimum mean square combining is employed in the receiver, the system is able to adjust the equalization gain coefficients in every iterative stage. In addition, both the soft in soft out (SISO) inverse bit mapper and simplified SISO bit mapper are used in the receiver to avoid the problems of high complexity, long latency and error amplification caused by the decoder in every iteration step. The partial cancellation is considered to further improve the receiver performance as well. The simulations are carried out in a realistic multi-user environment: modified 802.16 Stanford University Interim (SUI)

Table 3.2: Main parameters used in simulations

\begin{tabular}{|c|c|c|c|}
\hline Signal bit rate & $4 \mathrm{MHz}$ & $\begin{array}{c}\text { Modulation } \\
\text { scheme }\end{array}$ & 256 QAM \\
\hline $\begin{array}{c}\text { Number of data } \\
\text { sub-carriers }\end{array}$ & 256 & Cyclic prefix & 16 us \\
\hline FFT length & 256 & Channel model & $\begin{array}{c}\text { Modified SUI } \\
\text { with AWGN }\end{array}$ \\
\hline $\begin{array}{c}\text { Maximum } \\
\text { Doppler } \\
\text { Frequency }\end{array}$ & $120 \mathrm{~Hz}$ & $\begin{array}{c}\text { OFDM symbol } \\
\text { period }\end{array}$ & 64 us \\
\hline $\begin{array}{c}\text { Spreading } \\
\text { Sequences }\end{array}$ & $\begin{array}{c}\text { Walsh } \\
\text { codes }\end{array}$ & $\begin{array}{c}\text { Turbo code } \\
\text { rate }\end{array}$ & 0.5 \\
\hline
\end{tabular}


channel model. Both analysis and simulations indicate that our proposed receiver achieves a considerably better performance with a low complexity structure compared with traditional systems. 


\section{CHAPTER IV}

\section{INTERFERENCE ANALYSIS FOR}

\section{MULTICARRIER SYSTEMS}

In this chapter, the interference effects for multicarrier systems are considered. We begin with the general OFDM systems in the section 4.1. The special problems in MC-CDMA system are then discussed in the section 4.2.

\subsection{Performance Analysis of Interference for OFDM Systems}

Since OFDM is a very promising candidate for the core technique of next-generation wireless communication systems, it is necessary to evaluate its performance under interference over fading channels. The interference can be unintentional, such as cochannel interference, or intentional, which is deliberately injected to disrupt the opponent's communications. This part focuses on the performance analysis of OFDM under the various interference models. There are some works done in this area. In [46], the performance of OFDM communication in the presence of partial-band interference is presented. The anti-interference property of clustered OFDM has been investigated in [47], and [48] gives a detailed study about the effect of partial band interference on OFDM systems. Despite of all the works mentioned, a comprehensive study about the effects of different interference models for OFDM systems has not been carried out. By comparing the Bit Error Rate (BER) performance of different interference models, the most effective interference model can be identified under various channel conditions. 
This is very critical for both intentional interference injecting and anti-interference applications for OFDM systems.

This part evaluates the BER performance of different interference models, including Barrage Noise Interference (BNI), Partial Band Interference (PBI) and Multitone Interference (MTI) in time-correlated Rayleigh fading channel with AWGN. In addition, two novel interference injecting methods - optimal-fraction PBI and optimal-fraction MTI for OFDM systems are proposed with detailed theoretical analysis. The theoretical and simulation results show that the most effective interference injecting strategy for OFDM systems is the optimal-fraction MTI since it can make interference effect better obviously through a simple way.

This part is organized as follows. In Section 4.1.1, a brief overview of OFDM system model is presented. Section 4.1.2 details the different interference models and their analytical BER forms in OFDM systems. Simulation results and related analysis are shown in Section 4.1.3. Finally, the concluding remarks are given in Section 4.1.4.

\subsubsection{OFDM system and channel model}

The overview of OFDM system model is illustrated in Figure 4.1. In this chapter, we use Binary Phase Shift Keying (BPSK) and Differential Binary Phase Shift Keying (DBPSK) as our signal mapping methods. Higher order modulation techniques can be used as well, but these two are sufficient for us to analyze the essence of the problem. When applying DBPSK to OFDM systems, Frequency Domain Differential Demodulation (FDDD) is used rather than Time Domain Differential Demodulation (TDDD) since FDDD outperforms TDDD in frequency-nonselective fading channel [49]. The Cyclic Prefix is 


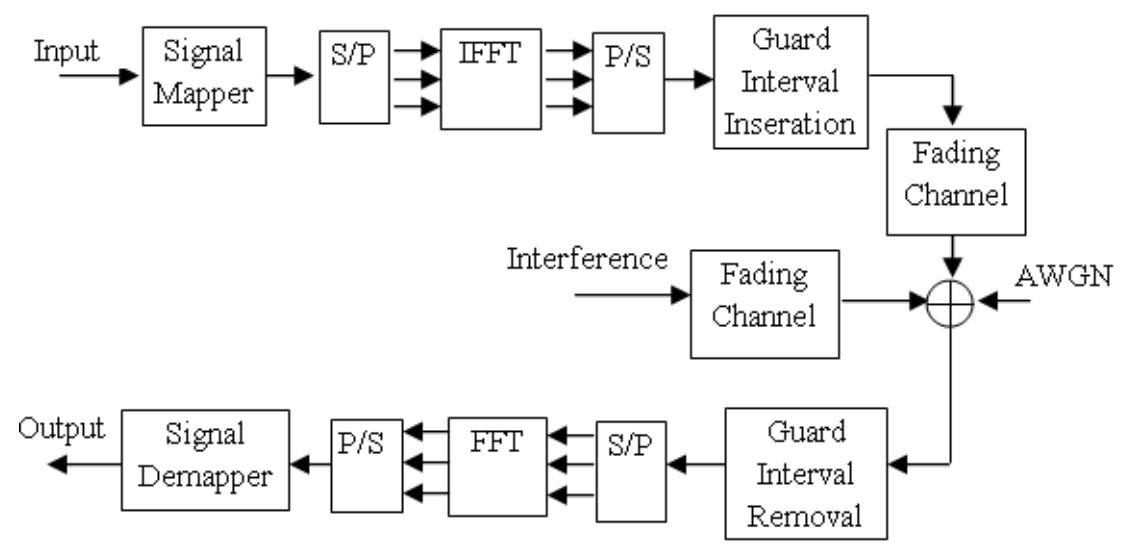

Figure 4.1. OFDM System Model

used as Guard Interval to eliminate ISI between the data blocks since samples of the channel output affected by this ISI can be discarded without any loss relative to the original information sequence [45].

The channel is modeled as a flat-fading Rayleigh channel. For every sub-carrier in OFDM systems, its bandwidth is relatively small compared with the bandwidth of the channel, so it is reasonable to make this flat-fading assumption. Based on the method in [55], we construct a time-correlated flat-fading Rayleigh model, in which the channel has a Rayleigh-distributed envelope and uniform phase, and the two are mutually independent. We assume the system is coherent, so the phase can always be estimated perfectly. Hence we neglect the phase variation of the channel. Considering the interference and the signal are both independently attenuated by the channel, we use two independent random variables to describe the interference channel power gain $\mathrm{G}_{\mathrm{J}}$ and the signal channel power gain $G_{s}$, which are given as

$$
\begin{aligned}
& G_{S}=\alpha^{2} \\
& G_{J}=\beta^{2}
\end{aligned}
$$


where $\alpha$ and $\beta$ are independent Rayleigh random variables with variances $\sigma_{S}^{2}$ and $\sigma_{J}^{2}$, respectively. Because the interference and signal are under the same channel environment, $\sigma_{S}$ and $\sigma_{J}$ can be regarded as the same value $\sigma$.

\subsubsection{The effects of various Interference for OFDM systems}

In this section we investigate several typical interference models. For every interference type, we first give the BER form under AWGN, then consider more complicated Rayleigh fading channel.

\section{A. BNI under $A W G N$}

Barrage Noise Interference (BNI) belongs to a broadband noise interference type. In this case, the interference interferes with the whole bandwidth by injecting a band-limited noise to the system. Its effect is the same as that of the AWGN noise, so the Power Spectrum Density (PSD) of total noise becomes:

$$
P S D_{N}=N_{0}+N_{J}
$$

where $N_{0}$ is the noise PSD of complex AWGN noise and $N_{J}$ is the PSD of complex BNI. Since the OFDM system performs no differently from conventional serial systems under the AWGN [53], the BER for BPSK and DBPSK is given as:

$$
\begin{gathered}
P_{B P S K}=Q\left(\sqrt{\frac{2 E_{b}}{N_{0}+N_{J}}}\right) \\
P_{D B P S K}=\frac{1}{2} \exp \left(\frac{-E_{b}}{N_{0}+N_{J}}\right)
\end{gathered}
$$

where $E_{b}$ is the average energy-per-bit of OFDM signal. 


\section{B. BNI under Rayleigh fading channel with AWGN}

In Rayleigh fading channel, the effective energy-per-bit becomes $G_{S} E_{b}$ and the effective PSD of the BNI becomes $G_{J} N_{J}$. Under the assumption that the time correlation coefficient of the channel is close to 1 , the time-varying property of the channel will not affect the analysis of the differential modulation. After simplifying, we get the BER

$$
\begin{gathered}
P_{B P S K}(\alpha, \beta)=Q\left(\sqrt{\frac{2 \alpha^{2} E_{b}}{N_{0}+\beta^{2} N_{J}}}\right) \\
P_{D B P S K}(\alpha, \beta)=\frac{1}{2} \exp \left(\frac{-\alpha^{2} E_{b}}{N_{0}+\beta^{2} N_{J}}\right)
\end{gathered}
$$

Since there are two Rayleigh random variables with the same variance $\sigma^{2}$, the average BER for BPSK and DPBSK can be expressed as

$$
\begin{gathered}
\overline{P_{B P S K}}=\int_{0}^{\infty} \int_{0}^{\infty} Q\left(\sqrt{\frac{2 \alpha^{2} E_{b}}{N_{0}+\beta^{2} N_{J}}}\right) \cdot \frac{\alpha \beta}{\sigma^{4}} \cdot \exp \left(\frac{-\alpha^{2}-\beta^{2}}{2 \sigma^{2}}\right) d \alpha d \beta \\
\overline{P_{D B P S K}}=\int_{0}^{\infty} \int_{0}^{\infty} \frac{1}{2} \exp \left(\frac{-\alpha^{2} E_{b}}{N_{0}+\beta^{2} N_{J}}\right) \cdot \frac{\alpha \beta}{\sigma^{4}} \cdot \exp \left(\frac{-\alpha^{2}-\beta^{2}}{2 \sigma^{2}}\right) d \alpha d \beta
\end{gathered}
$$

Certainly, the infinite upper limit of integration should be replaced by finite approximated value in numerical calculation.

\section{PBI under AWGN}

Partial Band Interference (PBI) is modeled as additive Gaussian noise with its power focusing on a portion of the entire bandwidth of the system. This strategy is considered more effective than BNI since the interference can use more power to interfere with certain specific bandwidth. We consider the best interference scenario: The interference 
signal bandwidth falls into that of the OFDM signal completely. The portion of interference signal bandwidth can be described by [46]

$$
\rho=\frac{W_{j}}{W_{s i g}}
$$

where $W_{j}$ is the bandwidth of the interference signal and $W_{\text {sig }}$ is the bandwidth of the OFDM signal. To calculate the BER, we consider two types of frequency bands: the interfered frequency bands and the uninterfered frequency bands. Given the average PSD of PBI $\mathrm{N}_{\mathrm{J}}$, the effective PSD of PBI in the first type of bands becomes $N_{J} / \rho$, and there is no interference at all in the second type of bands. Combing those two cases with (4.4) and (4.5), the BER for BPSK and DBPSK under PBI is given as

$$
\begin{gathered}
P_{B P S K}(\rho)=\rho \cdot Q\left(\sqrt{\frac{2 E_{b}}{N_{0}+N_{J} / \rho}}\right)+(1-\rho) \cdot Q\left(\sqrt{\frac{2 E_{b}}{N_{0}}}\right) \\
P_{D B P S K}(\rho)=\frac{\rho}{2} \cdot \exp \left(\frac{-E_{b}}{N_{0}+N_{J} / \rho}\right)+\left(\frac{1-\rho}{2}\right) \cdot \exp \left(\frac{-E_{b}}{N_{0}}\right)
\end{gathered}
$$

Since (4.11) and (4.12) depend on the value of $\rho$, it is necessary to find the optimal interference fraction $\rho^{*}$ so that the interference effect is maximized. Assuming the background AWGN is negligible compared to PBI and applying the following approximation for the $\mathrm{Q}$ function from [52].

$$
Q(x) \approx \exp \left(-\frac{x^{2}}{2}\right) /\left(1.64 x+\sqrt{0.76 x^{2}+4}\right)
$$

Eq. (4.11), (4.12) are transformed into

$$
\widehat{P}_{B P S K}(\rho)=\rho \frac{\exp (-S I R \cdot \rho)}{(1.64 \sqrt{2 S I R \cdot \rho}+\sqrt{1.52 \cdot S I R \cdot \rho+4})}
$$




$$
\widehat{P}_{D B P S K}(\rho)=\frac{\rho}{2} \exp (-S I R \cdot \rho)
$$

where SIR represents Signal-to-Interference Ratio, which is equal to $E_{b} / N_{J}$. The optimal Interference fraction $\rho^{*}$ can be obtained by maximizing (4.14) and (4.15) with respect to $\rho$ for a given $S I R$

$$
\begin{gathered}
\rho_{B P S K}^{*}=\underset{\rho}{\arg \max } \hat{P}_{B P S K}(\rho) \\
\rho_{D B P S K}^{*}=\underset{\rho}{\arg \max } \hat{P}_{D B P S K}(\rho) \\
\text { Interference fraction constraint }: 0 \leq \rho \leq 1
\end{gathered}
$$

Let us take the partial derivative of (4.14) and (4.15) with respect to $\rho$ and set them to 0 to obtain optimal interference fractions. Here Newton-Raphson approximation is used to get numerical results. Like constrained control system, the optimal interference fraction will saturate whenever the boundary constraints $(0 \leq \rho \leq 1)$ are violated. In practice, the optimal values of interference fraction can be generated offline based on different SIR, and then stored in hardware or software as a table. By looking up this table, the interference generator can maintain optimal performance for every value of the SIR. In this chapter, this special PBI based on optimal interference fraction table is named as optimal-fraction PBI.

\section{PBI under Rayleigh fading channel with AWGN}

For the time-correlated Rayleigh fading channel, following the same steps as before, the average BER for BPSK and DPBSK becomes

$$
\overline{P_{B P S K}}=\int_{0}^{\infty} \int_{0}^{\infty}\left(\rho \cdot Q\left(\sqrt{\frac{2 \alpha^{2} E_{b}}{N_{0}+\beta^{2} N_{J} / \rho}}\right)+(1-\rho) \cdot Q\left(\sqrt{\frac{2 \alpha^{2} E_{b}}{N_{0}}}\right)\right) \frac{\alpha \beta}{\sigma^{4}} \cdot \exp \left(\frac{-\alpha^{2}-\beta^{2}}{2 \sigma^{2}}\right) d \alpha d \beta
$$




$$
\overline{P_{D B P S K}}=\int_{0}^{\infty} \int_{0}^{\infty}\left(\frac{\rho}{2} \cdot \exp \left(\frac{-\alpha^{2} E_{b}}{N_{0}+\beta^{2} N_{J} / \rho}\right)+\left(\frac{1-\rho}{2}\right) \cdot \exp \left(\frac{-\alpha^{2} E_{b}}{N_{0}}\right)\right) \frac{\alpha \beta}{\sigma^{4}} \cdot \exp \left(\frac{-\alpha^{2}-\beta^{2}}{2 \sigma^{2}}\right) d \alpha d \beta
$$

Here we do not consider the optimization of interference fraction, since this requires a complicated algorithm in a time-varying Rayleigh fading channel. It is not realistic to do so just for small improvement in interference effect. Instead we introduce an empirical equation to get the approximation solution. As Rayleigh fading channel is a special form of Rician fading channel, the commonly used Rician fading amplitude with unit meansquared value is defined as [50]

$$
\gamma_{i}=\sqrt{\frac{\left(x_{i}+\sqrt{2 K}\right)^{2}+y_{i}^{2}}{2(K+1)}}
$$

where $x_{i}$ and $y_{i}$ are samples of zero-mean stationary Gaussian random processes with variance $\sigma_{0}^{2}=1$, which can be generated by the method in [55]. The best and worst-case Rician fading channels associated with $K$-factors of $K=\infty$ and $K=0$ are the Gaussian and Rayleigh channels with strong Line Of Sight (LOS) and without LOS path, respectively. From the previous section, it is easy to get the optimal interference fractions in AWGN channels: $\rho_{B P S K_{-} A W G N}^{*}$ and $\rho_{D B P S K_{-} A W G N}^{*}$ (unconstrained values from Eq. (4.16) and (4.17). Based on these values, we can get the optimal interference fractions in Rician channel by empirical fit as

$$
\begin{gathered}
\rho_{\text {BPSK_RICIAN }}^{*}=\left(1+e^{-0.0123 K^{2}-0.148 K+2.20}\right) \rho_{B P S K_{-} A W G N}^{*} \\
\rho_{\text {DBPSK_RICIAN }}^{*}=\left(1+e^{-0.0023 K^{2}-0.2543 K+2.08}\right) \rho_{D B P S K_{-} A W G N}^{*}
\end{gathered}
$$

(Interference fraction constraint: $0 \leq \rho \leq 1$ ) 


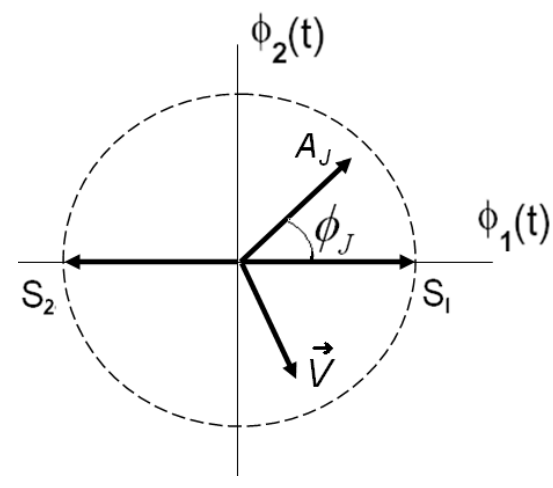

Figure 4.2. MTI vector space

\section{E. MTI under AWGN}

Multitone Interference (MTI) divides its total power into q distinct, equal power, random phase tones. Every interference tone can be modeled as

$$
J(t)=A_{J} e^{j\left(2 \pi f f_{J} t+\phi_{J}\right)}
$$

where $\phi_{J}$ is the random phase, which is uniformly distributed over $[0,2 \pi] . A_{J}$ and $f_{J}$ are the amplitude and frequency, respectively. We assume that those $q$ interference tones are perfectly aligned with $q$ sub-carriers of the OFDM system. Then the portion of interference signal bandwidth is defined as

$$
\rho=q / M
$$

where $M$ is the number of FFT points. After FFT in receiver block, in signal space, the interference signal, which has fixed length $A_{J}$ and random phase $\phi_{J}$, is added to original signal $\left(s_{1}\right.$ or $S_{2}$ ) as a vector (Figure 4.2), and AWGN noise $\vec{V}$ is another vector added to them. Projecting the compound signal onto $\phi_{1}$ axis, we get

$$
\begin{gathered}
S_{\phi 1, r 1}=A_{J} \cos \left(\phi_{J}\right)+\sqrt{E_{b}}+V_{\phi 1} \\
S_{\phi 1, r 2}=A_{J} \cos \left(\phi_{J}\right)-\sqrt{E_{b}}+V_{\phi 1}
\end{gathered}
$$


where $S_{\phi 1, r 1}$ and $S_{\phi 1, r 2}$ represent the $\phi_{1}$ axis projection of received signals (corresponding to $S_{1}$ and $S_{2}$, respectively), and $V_{\phi 1}$ is the AWGN noise $\vec{V}$ 's $\phi_{1}$ axis projection. Because the error probabilities for $S_{1}$ and $S_{2}$ are equal, only $P_{e_{s}}$ needs to be calculated. Hence the BER for BPSK with MTI is given as

$$
P_{B P S K_{-} M T J}=P_{e_{S 1}}=\operatorname{Pr}\left(S_{\phi 1, r 1}<0\right)
$$

Here we have two random variables: one is $\phi_{J}$, which is uniformly distributed over $[0,2 \pi]$; the other is $V_{\phi 1}$, which satisfies the Gaussian distribution with $N\left(0, N_{0} / 2\right)$. Now we define $Y=A_{J} \cos \left(\phi_{J}\right)+\sqrt{E_{b}}, X=V_{\phi 1}$ and $W=X+Y$. Then

$$
P_{B P S K_{-} M T J}=\operatorname{Pr}\left(S_{\phi 1, r 1}<0\right)=\operatorname{Pr}(W<0)
$$

The Probability Density Function (PDF) of $\cos (\theta)$ function is given in [51] as

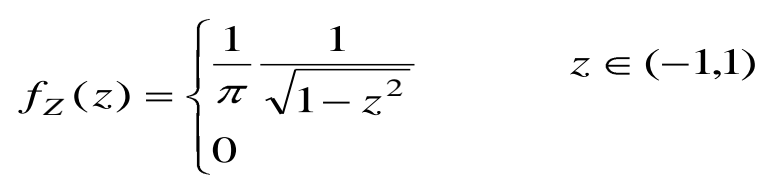

The PDF of Y can be represented as

$$
f_{Y}(y)=\frac{1}{\pi A_{J} \sqrt{1-\left(\frac{y-\sqrt{E_{b}}}{A_{J}}\right)^{2}}}
$$

where y should satisfy $\sqrt{E_{b}}-A_{J}<y<\sqrt{E_{b}}+A_{J}$

Given the PDF of $\mathrm{X}$, the PDF of $\mathrm{W}$ is

$$
\begin{aligned}
& f_{W}(w)=\int_{-\infty}^{+\infty} f_{X}(w-y) f_{Y}(y) d y \\
& =\int_{\sqrt{E_{b}-A_{J}}}^{\sqrt{E_{b}}+A_{J}} \frac{1}{\sqrt{\pi N_{0}}} \exp \left(-\frac{(w-y)^{2}}{N_{0}}\right) \frac{1}{\pi A_{J} \sqrt{1-\left(\frac{y-\sqrt{E_{b}}}{A_{J}}\right)^{2}}} d y
\end{aligned}
$$


Then $A_{J}$ is the only unknown variable in (4.31), which can be obtained simply through the following equation

$$
A_{J}=\sqrt{\frac{E_{b}}{\rho S I R}}
$$

Based on the result of (4.31), we can get $P_{B P S K_{-} M T J}$ from (4.28) easily. Thus the BER for BPSK is given as

$$
\begin{aligned}
& P_{B P S K}\left(E_{b}, N_{0}, \rho, S I R\right)=\rho \cdot P_{B P S K_{-} M T J}+(1-\rho) Q\left(\sqrt{\frac{2 E_{b}}{N_{0}}}\right) \\
& =\rho \int_{-\infty}^{0} f_{W}(w) d w+(1-\rho) Q\left(\sqrt{\frac{2 E_{b}}{N_{0}}}\right)
\end{aligned}
$$

If the AWGN noise is negligible, we can get

$$
P_{B P S K}\left(E_{b}, \rho, S I R\right)=P_{e s 1}= \begin{cases}\frac{\rho}{\pi}\left(\frac{\pi}{2}-\arcsin (\sqrt{\rho S I R})\right) & \sqrt{\rho S I R}<1 \\ 0 & \end{cases}
$$

For DBPSK, let $z(k-1)$ and $z(k)$ be the reference and received symbol vectors respectively. The variable $D$ is given as

$$
\begin{aligned}
& D=\operatorname{Re}\left(z(k) z^{*}(k-1)\right)= \\
& \operatorname{Re}\left(\left(A_{J} e^{j \phi_{J}(k)}+s(k)+\vec{V}(k)\right)\left(A_{J} e^{-j \phi_{J}(k-1)}+s^{*}(k-1)+\vec{V}^{*}(k-1)\right)\right)
\end{aligned}
$$

where $s(k)$ and $s(k-1)$ are transmitted constellations at time $k$ and $k-1 . D$ is used as differential detector to decide which symbol was transmitted. Due to symmetry, we can assume a given phase difference zero to compute the error probability [45], so $s(k)$ and $s(k-1)$ can be specified as $\sqrt{E_{b}}$. Hence

$$
\begin{aligned}
& D=\operatorname{Re}\left(z(k) z^{*}(k-1)\right)= \\
& \operatorname{Re}\left(\left(A_{J} e^{j \phi_{J}(k)}+\sqrt{E_{b}}+\vec{V}(k)\right)\left(A_{J} e^{-j \phi_{J}(k-1)}+\sqrt{E_{b}}+\vec{V}^{*}(k-1)\right)\right)
\end{aligned}
$$


If $D$ is less than 0 , then a decision error is made. That is, the BER equals to $\operatorname{Pr}(D<0)$ when MTI exists. However, the probability of $D$ is difficult to calculate from (4.36), subsequently some approximations are necessary. For $S I R \gg S N R\left(\mathrm{E}_{\mathrm{b}} / \mathrm{N}_{0}\right)$, we can neglect some small items in (4.36), and get

$$
\begin{aligned}
& D \approx E_{b}+A_{J} \sqrt{E_{b}}\left(\cos \left(\phi_{J}(k)\right)+\cos \left(\phi_{J}(k-1)\right)\right)+\sqrt{E_{b}}\left(V_{\phi 1}(k)+V_{\phi 1}(k+1)\right) \\
& +A_{J}^{2}\left(\cos \left(\phi_{J}(k)-\phi_{J}(k-1)\right)\right)
\end{aligned}
$$

Dividing (4.37) by $\sqrt{E_{b}}$ yields

$$
\begin{aligned}
& \bar{D} \approx \sqrt{E_{b}}+A_{J}\left(\cos \left(\phi_{J}(k)\right)+\cos \left(\phi_{J}(k-1)\right)\right)+V_{\phi 1}(k)+V_{\phi 1}(k-1) \\
& +\frac{A_{J}^{2}}{\sqrt{E_{b}}}\left(\cos \left(\phi_{J}(k)-\phi_{J}(k-1)\right)\right)
\end{aligned}
$$

Compared with the case of coherent BPSK (4.25), there are five noise terms instead of two. Approximately, the MTI is $2+1 / \sqrt{\rho S I R}$ times larger than that of BPSK and AWGN is 2 times larger than that of BPSK, which gives a simple way of getting differential modulation BER from coherent modulation BER in Eq. (4.33).

$$
P_{D B P S K}\left(E_{b}, N_{0}, \rho, S I R\right)=P_{B P S K}\left(E_{b}, 2 N_{0}, \rho, \frac{S I R}{\left(2+\frac{1}{\sqrt{\rho S I R}}\right)}\right)
$$

This equation is valid only for $S I R>>S N R$. From simulation, we found that when $S I R$ is close to $S N R$, the simulation values of BER will deviate from theoretical values to some small values. In order to compensate this deviation, Eq. (4.39) was modified empirically to

$$
P_{D B P S K}\left(E_{b}, N_{0}, \rho, S I R\right)=P_{B P S K}\left(E_{b}, N_{0}, \rho, \frac{S I R}{\left(2+\frac{1}{\sqrt{\rho S I R}}-0.6\right)}\right)
$$

which shows good results in the simulation. 
In MTI, optimal interference fraction should also be considered, so that we get the optimal-fraction MTI. Again, assuming the background AWGN is negligible, from (4.34) following the same process as PBI, we can get

$$
\frac{1}{\pi}\left(\frac{\pi}{2}-\arcsin \left(\sqrt{\rho_{B P S K}^{*} \cdot S I R}\right)\right)+\frac{\rho_{B P S K}^{*}}{\pi} \frac{-0.5 \cdot S I R}{\left.\sqrt{\rho_{B P S K}^{*} \cdot S I R\left(1-\rho_{B P S K}^{*} \cdot S I R\right.}\right)}=0
$$

Solving it with different SIR will generate MTI interference fraction optimization table of BPSK. For DBPSK, the optimal interference fraction can be obtained from simulation results. The optimization tables of BPSK and DBPSK will be used by optimal-fraction multitone interference generator to achieve optimal performance.

\section{F. MTI under Rayleigh fading channel with AWGN}

Similar as before, the average BER for BPSK and DBPSK for MTI under Rayleigh fading channel with AWGN noise is

$$
\begin{gathered}
\overline{P_{B P S K}}=\int_{0}^{\infty} \int_{0}^{\infty}\left(P_{B P S K}\left(\alpha^{2} E_{b}, N_{0}, \rho, \alpha^{2} \operatorname{SIR} / \beta^{2}\right)\right) \frac{\alpha \beta}{\sigma^{4}} \cdot \exp \left(\frac{-\alpha^{2}-\beta^{2}}{2 \sigma^{2}}\right) d \alpha d \beta \\
\overline{P_{D B P S K}}=\int_{0}^{\infty} \int_{0}^{\infty}\left(P_{D B P S K}\left(\alpha^{2} E_{b}, N_{0}, \rho, \alpha^{2} S I R / \beta^{2}\right) \frac{\alpha \beta}{\sigma^{4}} \cdot \exp \left(\frac{-\alpha^{2}-\beta^{2}}{2 \sigma^{2}}\right) d \alpha d \beta\right.
\end{gathered}
$$

where $P_{B P S K}\left(\alpha^{2} E_{b}, N_{0}, \rho, \alpha^{2} S I R / \beta^{2}\right)$ is derived from (4.33), and when you calculate it, you should change all $E_{b}$ and $S I R$ to $\alpha^{2} E_{b}, \alpha^{2} S I R / \beta^{2}$ correspondingly. Eq. (4.40) gives the value of $P_{D B P S K}\left(\alpha^{2} E_{b}, N_{0}, \rho, \alpha^{2} S I R / \beta^{2}\right)$. Here $E_{b}$ and $S I R$ should be changed as well. Also, given the MTI optimal interference fractions in AWGN channels: $\rho_{B P S K_{-} A W G N}^{*}$ and 
$\rho_{D B P S K_{-} A W G N}^{*}$ (unconstrained values), the optimal interference fractions in Rician channel can be obtained empirically as

$$
\begin{gathered}
\rho_{\text {BPSK__RICIAN }}^{*}=\left(1+e^{-0.0187 K^{2}-0.3528 K+2.4}\right) \rho_{B P S K_{-} A W G N}^{*} \\
\rho_{D B P S K_{-} \text {RICIAN }}^{*}=\left(1+e^{-0.0131 K^{2}-0.308 K+2.49}\right) \rho_{D B P S K_{-} A W G N}^{*}
\end{gathered}
$$

(Interference fraction constraint: $0 \leq \rho \leq 1$ )

\subsubsection{Simulation results and analysis}

In this section, the BER performance of different interference techniques for OFDM system is evaluated by the means of software simulation. Based on the 802.11a standard [54], the main parameters used in the simulation are summarized as Table 4.1. In this table, to simplify the problem, we use 64 as the number of sub-carriers instead of 52 in the 802.11a standard. Hence the occupied bandwidth is changed from $16.6 \mathrm{MHz}$ to 20 MH correspondingly.

Figure 4.3 shows the comparison between simulation results and theoretical results of all non-optimal interference types (BNI, the fixed-fraction PBI and the fixed-fraction MTI) in the Chapter. In the simulation, every test is repeated $200-1000$ times to eliminate the

Table 4.1. Main parameters used in simulations.

\begin{tabular}{|c|c|c|c|}
\hline Signal bit rate & $20 \mathrm{MHz}$ & $\begin{array}{c}\text { Modulation } \\
\text { Scheme }\end{array}$ & BPSK/DBPSK \\
\hline $\begin{array}{c}\text { Number of } \\
\text { sub-carriers }\end{array}$ & 64 & $\begin{array}{c}\text { Cyclic } \\
\text { prefix }\end{array}$ & $0.8 \mathrm{us}$ \\
\hline FFT length & 64 & $\begin{array}{c}\text { Channel } \\
\text { model }\end{array}$ & $\begin{array}{c}\text { Rayleigh fading channel } \\
\text { with AWGN noise }\end{array}$ \\
\hline $\begin{array}{c}\text { Doppler } \\
\text { frequency }\end{array}$ & $40 \mathrm{~Hz}$ & $\begin{array}{c}\text { OFDM symbol } \\
\text { period }\end{array}$ & $3.2 \mathrm{us}$ \\
\hline $\begin{array}{c}\text { Signal } \\
\text { bandwidth }\end{array}$ & $20 \mathrm{MHz}$ & $\begin{array}{c}\text { Interference } \\
\text { bandwidth }\end{array}$ & $\begin{array}{c}\text { Depend on different } \\
\text { interference techniques }\end{array}$ \\
\hline
\end{tabular}


fluctuations caused by intrinsic random nature of the OFDM communication system. It is shown that the simulation results of all non-optimal interference types are in agreement with the analytical prediction perfectly under AWGN. On the contrary, in Rayleigh fading channel, there are some small deviations between simulation results and theoretical values. They are caused by precision errors of numerical integration (for all non-optimal interference types) and equation approximation (only for DBPSK of MTI). All these deviations are less than $10 \%$, therefore they are acceptable.

To verify the optimization process about PBI and MTI, optimal Interference fraction data is listed as Table 4.2, in which $S N R$ is fixed to $20 \mathrm{db}$ and $S I R$ is varied from $-2 \mathrm{db}$ to $10 \mathrm{db}$. We show both analytical predictions and simulated values for PBI and MTI except MTI for DBPSK, for which only simulated values are shown since precise theoretical equation is hard to obtain. Since 64-FFT is used in the proposed OFDM system, every $\rho^{*}$ has been

Table 4.2. Optimal interference fraction $(\mathrm{SNR}=20 \mathrm{~dB})$

\begin{tabular}{llllllll}
\hline & $\rho *$ & $\rho *$ & $\rho *$ & $\rho *$ & $\rho *$ & $\rho *$ & $\rho *$ \\
SIR & PBI & PBI & PBI & PBI & MTI & MTI & MTI \\
{$[\mathrm{dB}]$} & $\mathbf{B P S K}$ & $\mathrm{BPSK}$ & \multicolumn{2}{l}{ DBPSK } & DBPSK BPSK & BPSK & DBPSK \\
& $\mathbf{( A )}$ & $(\mathrm{S})$ & $\mathbf{( A )}$ & $(\mathrm{S})$ & $\mathbf{( A )}$ & $(\mathrm{S})$ & $(\mathrm{S})$ \\
\hline-2 & $\mathbf{1}$ & $62 / 64$ & $\mathbf{1}$ & $62 / 64$ & $\mathbf{1}$ & 1 & 1 \\
-1 & $\mathbf{5 7 / 6 4}$ & $57 / 64$ & $\mathbf{1}$ & 1 & $\mathbf{5 1 / 6 4}$ & $48 / 64$ & $63 / 64$ \\
0 & $\mathbf{4 5 / 6 4}$ & $46 / 64$ & $\mathbf{1}$ & $61 / 64$ & $\mathbf{4 0 / 6 4}$ & $41 / 64$ & $55 / 64$ \\
1 & $\mathbf{3 6 / 6 4}$ & $35 / 64$ & $\mathbf{5 1 / 6 4}$ & $52 / 64$ & $\mathbf{3 2 / 6 4}$ & $32 / 64$ & $43 / 64$ \\
2 & $\mathbf{2 9 / 6 4}$ & $31 / 64$ & $\mathbf{4 0 / 6 4}$ & $41 / 64$ & $\mathbf{2 5 / 6 4}$ & $24 / 64$ & $32 / 64$ \\
3 & $\mathbf{2 3 / 6 4}$ & $26 / 64$ & $\mathbf{3 2 / 6 4}$ & $32 / 64$ & $\mathbf{2 0 / 6 4}$ & $20 / 64$ & $27 / 64$ \\
4 & $\mathbf{1 8} / \mathbf{6 4}$ & $21 / 64$ & $\mathbf{2 5 / 6 4}$ & $25 / 64$ & $\mathbf{1 6 / 6 4}$ & $15 / 64$ & $21 / 64$ \\
5 & $\mathbf{1 4 / 6 4}$ & $15 / 64$ & $\mathbf{2 0 / 6 4}$ & $21 / 64$ & $\mathbf{1 3 / 6 4}$ & $12 / 64$ & $16 / 64$ \\
6 & $\mathbf{1 1 / 6 4}$ & $11 / 64$ & $\mathbf{1 6 / 6 4}$ & $17 / 64$ & $\mathbf{1 0 / 6 4}$ & $11 / 64$ & $12 / 64$ \\
7 & $\mathbf{9 / 6 4}$ & $9 / 64$ & $\mathbf{1 3 / 6 4}$ & $14 / 64$ & $\mathbf{9 / 6 4}$ & $8 / 64$ & $10 / 64$ \\
8 & $\mathbf{7 / 6 4}$ & $7 / 64$ & $\mathbf{1 0 / 6 4}$ & $10 / 64$ & $\mathbf{6 / 6 4}$ & $7 / 64$ & $8 / 64$ \\
9 & $\mathbf{6 / 6 4}$ & $6 / 64$ & $\mathbf{8 / 6 4}$ & $8 / 64$ & $\mathbf{5 / 6 4}$ & $5 / 64$ & $6 / 64$ \\
10 & $\mathbf{5 / 6 4}$ & $4 / 64$ & $\mathbf{6 / 6 4}$ & $7 / 64$ & $\mathbf{4 / 6 4}$ & $4 / 64$ & $5 / 64$ \\
\hline And
\end{tabular}

$A$ : Analytical values, $S$ : Simulated values

This table is generated under AWGN channel without channel fading 
rounded to the closest integer multiple of $1 / 64$. In Table 4.2, the bold part is the analytical prediction and its right side is the corresponding simulated results. The biggest error between them is less than $5 \%$, which validates the correctness of the analytical model.

The comparison between the optimal-fraction interference and the fixed-fraction interference of PBI and MTI is shown in Figure 4.4. It is revealed that under AWGN and Rayleigh fading channel, the optimal-fraction interference always gives the best interference effect. Also it is found that the 0.9-fixed fraction interference almost gives the best interference effect in Rayleigh fading channel. In fact, in deep fading channel, the interference power should be distributed to the whole bandwidth to gain the best interference effect, so the optimal-fraction Interference degrades to whole bandwidth Interference in this case. In general, the optimal-fraction interference gives us a simple way to obtain good interference effect under various channel conditions.

Finally, optimal-fraction MTI and optimal-fraction PBI are compared in Figure 4.5 and Figure 4.6. Under various channel conditions shown in the Figure 4.5 and Figure 4.6, the optimal-fraction MTI clearly outperforms the optimal-fraction PBI for BPSK and DBPSK. The advantage of the optimal-faction MTI is most obvious in AWGN channel. As the channel condition gets worse, the advantage dies down gradually (See the Rician channel with $K=5$ and 10 in Figure 4. 6). In worst fading case - Rayleigh fading channel, the optimal-fraction MTI still outperforms the optimal-fraction PBI with a small advantage. Hence it is reasonable to believe that the optimal-fraction MTI can be used to obtain improved Interference effect under different channel conditions with low complexity. 


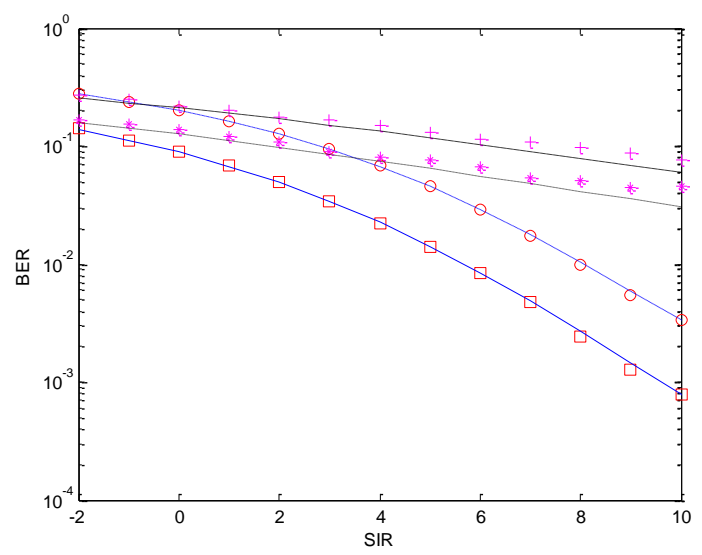

(a) BNI under AWGN and Rayleigh channel

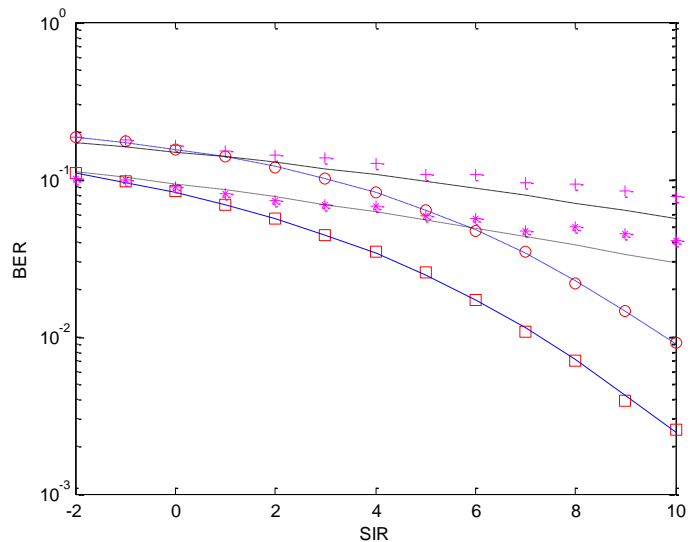

(b) PBI under AWGN and Rayleigh channel $(\rho=0.5)$

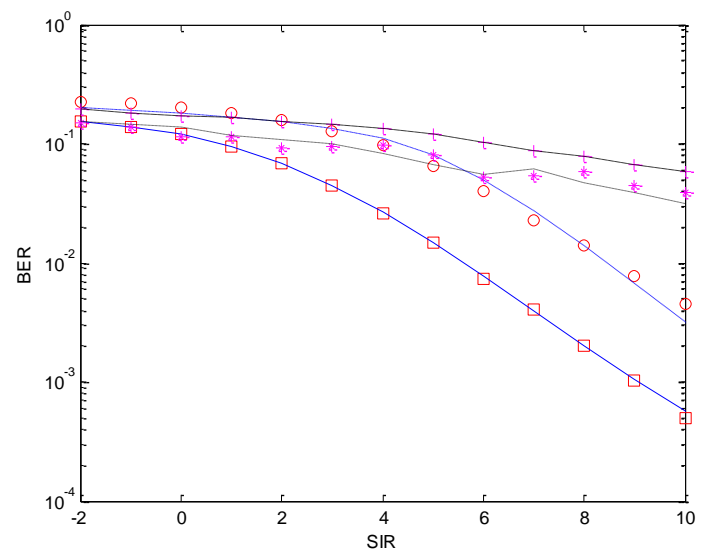

(c) MTI under AWGN and Rayleigh channel $(\rho=0.5)$

\begin{tabular}{|c|c|c|}
\hline- & $\begin{array}{l}\text { Analytical BER of BPSK (AWGN) } \\
\text { Analytical BER of DBPSK (AWGN) }\end{array}$ & $\begin{array}{l}--- \text { : Analytical BER of BPSK (Rayleigh) } \\
--- \text { : Analytical BER of DBPSK (Rayleigh) }\end{array}$ \\
\hline$\square$ & ed BER of BPSK (AWGN) & * : Simulated BER of BPSK (Rayleigh) \\
\hline o & : Simulated BER of DBPSK (AWGN) & $+:$ Simulated BER of DBPSK (Rayleigh) \\
\hline
\end{tabular}

Figure 4. 3. Comparison between simulation results and theoretical values of all interference strategies (SNR is fixed to $10 \mathrm{~dB}$ ) 


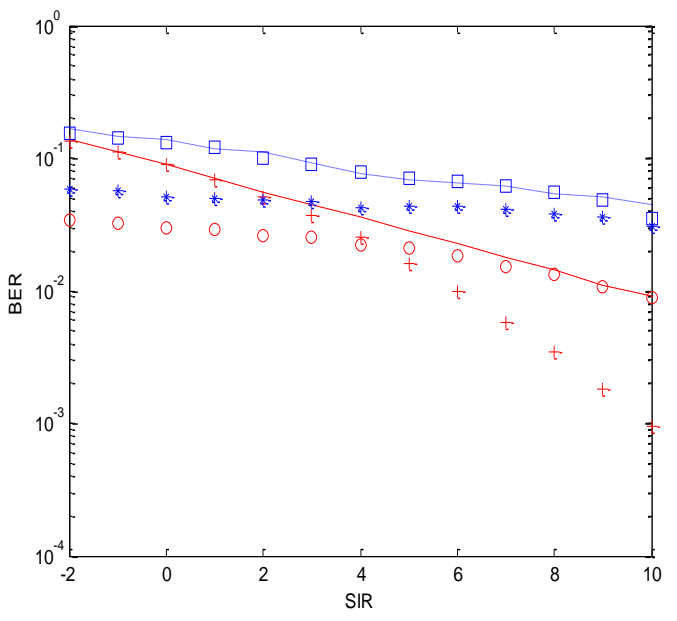

(a) PBI for BPSK

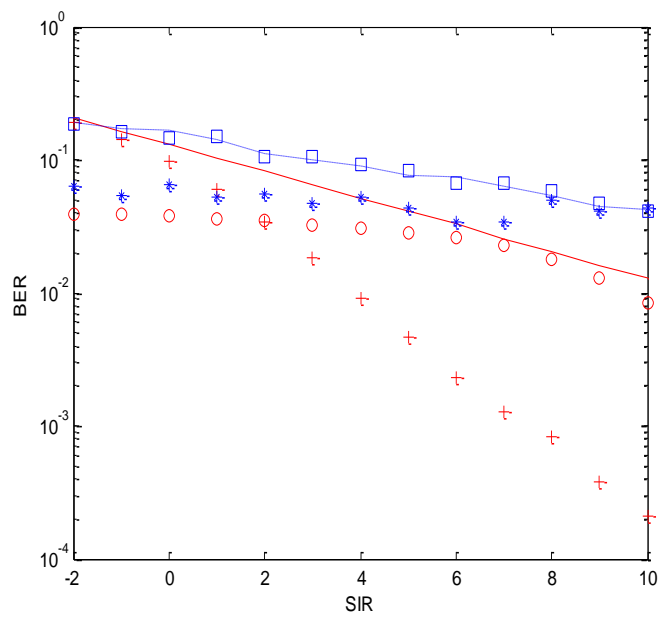

(c) MTI for BPSK

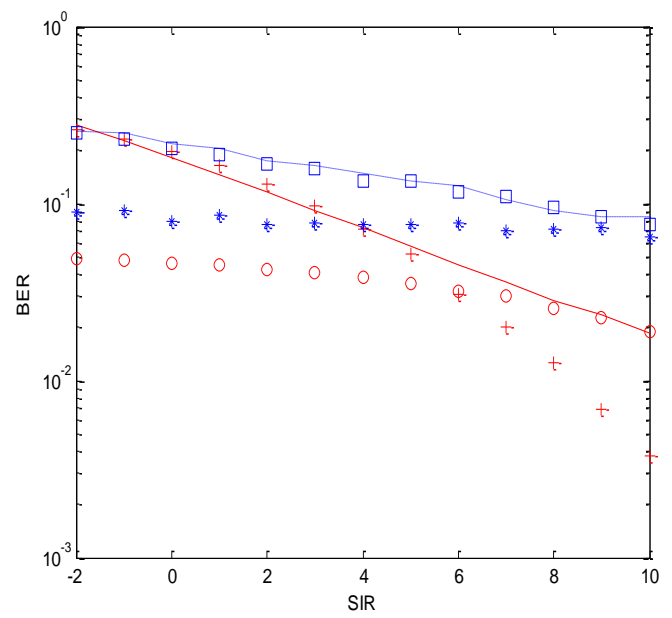

(b) PBI for DBPSK

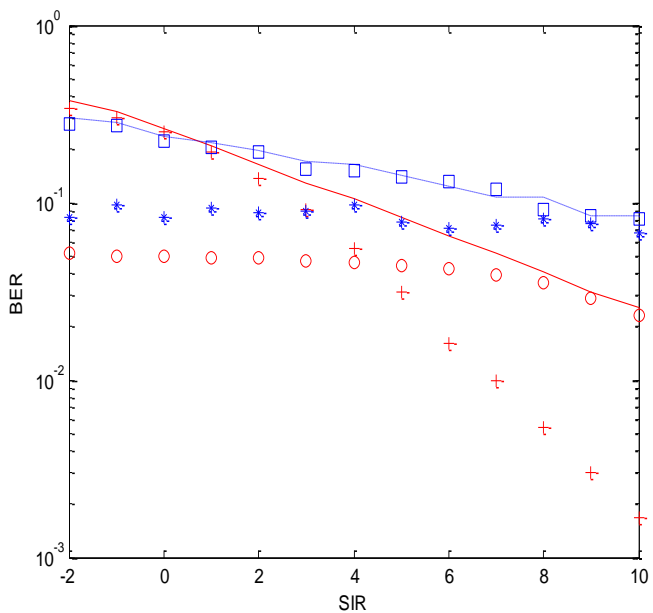

(d) MTI for DBPSK

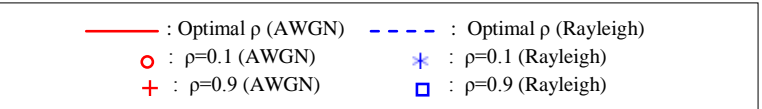

Figure 4. 4. Comparison between optimal-fraction interference and fixed-fraction interference of PBI and MTI (SNR is fixed to $10 \mathrm{~dB}$ ) 


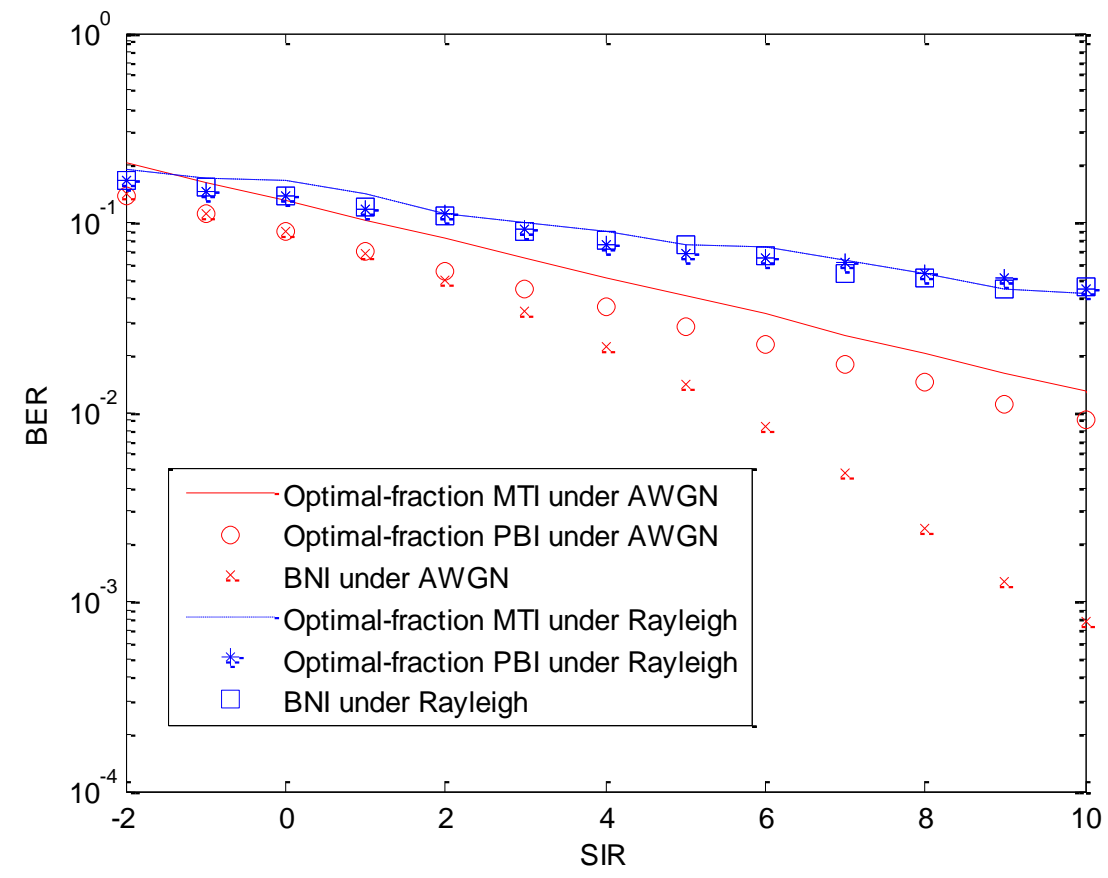


(a) Comparison for BPSK

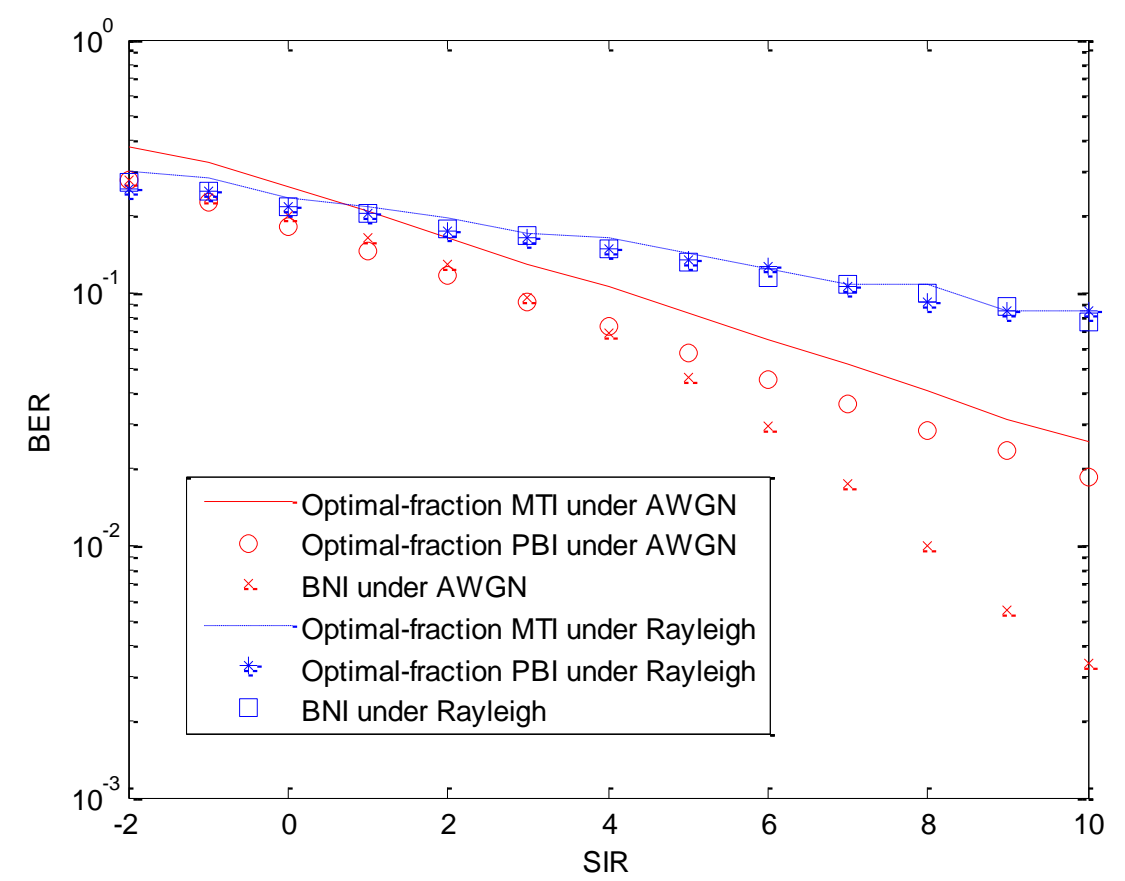

(b) Comparison for DBPSK

Figure 4. 5. Comparison of optimal-fraction MTI, optimal-fraction PBI and $\mathrm{BNI}$ ( $S N R$ is fixed to $10 \mathrm{~dB}$ in AWGN and Rayleigh channel) 


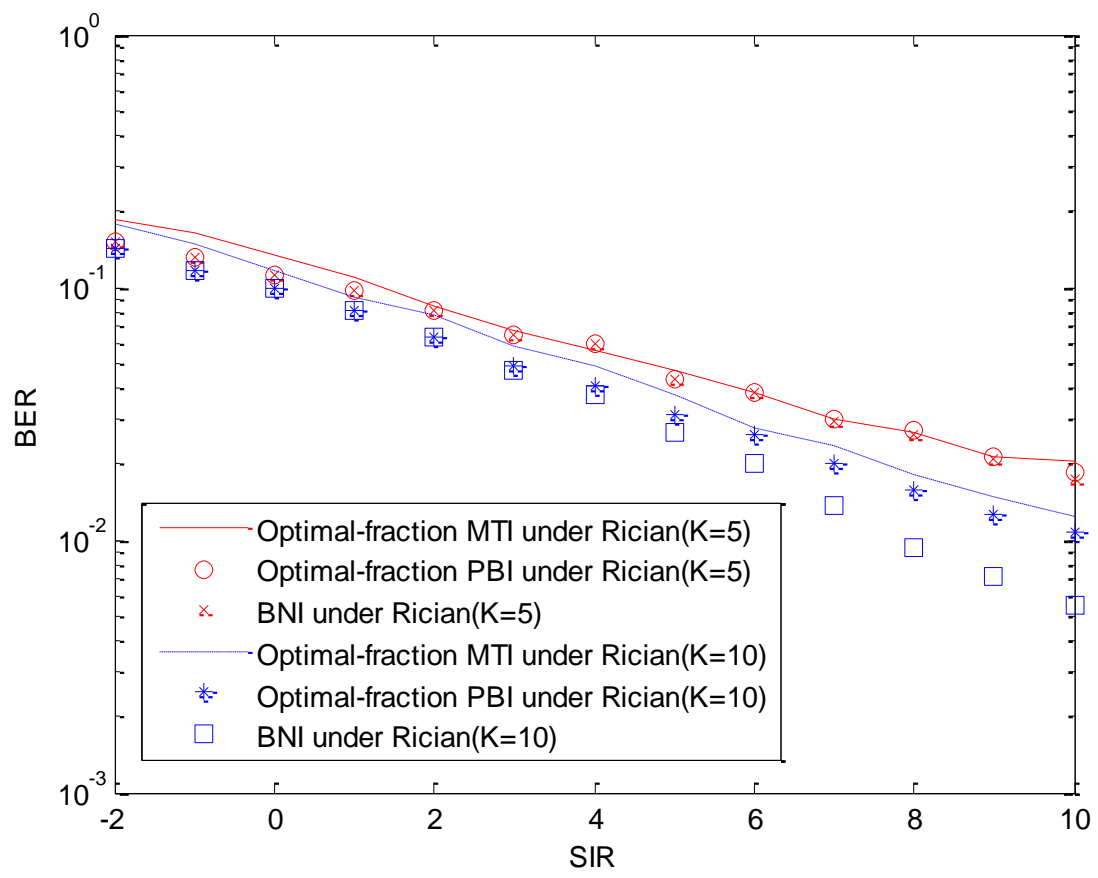

(a) Comparison for BPSK

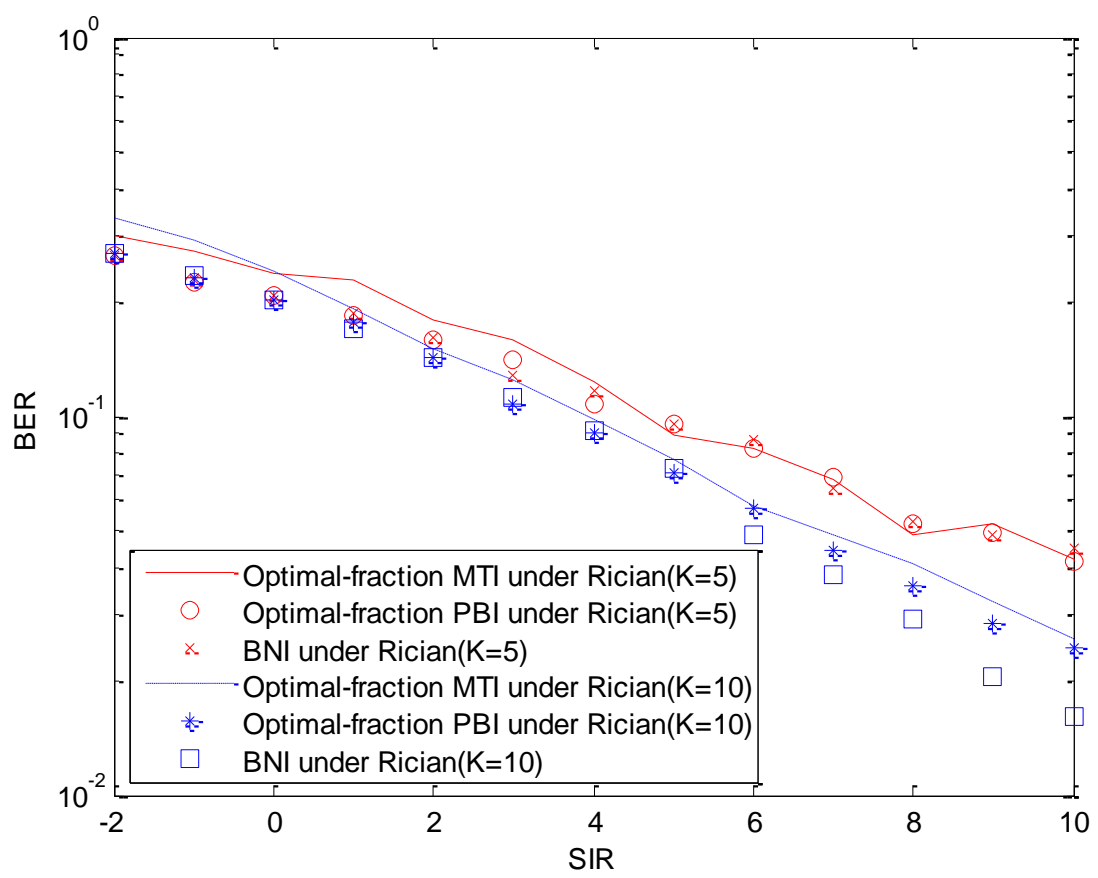

(b) Comparison for DBPSK

Figure 4. 6. Comparison of optimal-fraction MTI, optimal-fraction PBI and BNI ( $S N R$ is fixed to $10 \mathrm{~dB}$ in Rician channel, $\mathrm{K}=5$ and 10) 


\subsection{Performance Analysis of Interference for MC-CDMA Systems}

In this section, we analyze the interference for MC-CDMA systems. At the receiver, different combining techniques can be used. To simplify the analysis, we only consider the single user case with MRC and an AWGN channel is assumed as well.

\subsubsection{Partial-band interference}

First, let us consider the situation that we do not know the interference side information. Due to the property of MRC, the fraction of bandwidth interfered $\rho$, has no effect on the error probability, so Eqn. (4.4) and Eqn. (4.5) can be applied directly. We rewrite them here for the sake of clear illustrations:

$$
\begin{gathered}
P_{B P S K}=Q\left(\sqrt{\frac{2 E_{b}}{N_{0}+N_{J}}}\right) \\
P_{D B P S K}=\frac{1}{2} \exp \left(\frac{-E_{b}}{N_{0}+N_{J}}\right)
\end{gathered}
$$

With interference side information known, we can define the PSD of every subcarrier

$$
I_{k}=C_{k} N_{J} / \rho
$$

where $C_{k}$ is an interference state variable denoting whether or not the $k$ th subcarrier is interfered. When $C_{k}=0$, the $k$ th subcarrier is not interfered and when $C_{k}=1$, the $k$ th subcarrier is interfered. The total number of interfered subcarriers equals to $\sum_{k} C_{k}=\rho N$. The signal-to-interference plus noise ratio (SINR) for the $k$ th subcarrier is

$$
\operatorname{SINR}_{k}=\frac{E_{c}}{N_{0}+C_{k} N_{J} / \rho}
$$


where $E_{c}=E_{b} / N$ is the energy per subcarrier. Obviously according to the MRC principle in [45], the bit-error probability with BPSK and DBPSK is

$$
\begin{aligned}
& P_{B P S K}=Q\left(\sqrt{2 \sum_{k=0}^{N-1} \frac{E_{c}}{N_{0}+C_{k} N_{J} / \rho}}\right) \leq Q\left(\sqrt{2 \frac{E_{b}}{N_{0}+N_{J}}}\right) \\
& P_{D B P S K}=\frac{1}{2} \exp \left(-\sum_{k=0}^{N-1} \frac{E_{c}}{N_{0}+C_{k} N_{J} / \rho}\right) \leq \frac{1}{2} \exp \left(\frac{-E_{b}}{N_{0}+N_{J}}\right)
\end{aligned}
$$

with equality when all subcarriers are uniformly interfered. Hence the receiver can use interference side information to improve the performance.

\subsubsection{Pulse interference}

Broadband pulse interference transmits noise whose power is spread over the entire system bandwidth. However, the transmission only occurs for a fraction $\rho$ of the time. Here the $\rho$ is called duty cycle of the interference transmission and $0<\rho \leq 1$. This allows the inference to be transmitted with a power of

$$
J_{\text {peak }}=\frac{J}{\rho}
$$

where $J$ is the time-averaged power.

\subsubsection{Multi-tone interference}

If the AWGN noise is negligible, we can get the BER close form for ODFM systems

$$
P_{B P S K}\left(E_{b}, \rho, S I R\right)=P_{e s 1}= \begin{cases}\frac{\rho}{\pi}\left(\frac{\pi}{2}-\arcsin (\sqrt{\rho S I R})\right) & \sqrt{\rho S I R}<1 \\ 0 & \end{cases}
$$


Since the fraction of bandwidth interfered has no effect on the error probability, Eqn. (4.53) may be applied in MC-CDMA systems. With interference side information known, we can define the PSD of every subcarrier as (4.48)

$$
I_{k}=C_{k} N_{J} / \rho
$$

The signal-to-interference plus noise ratio (SINR) for the $k$ th subcarrier is

$$
\operatorname{SINR}_{k}=\frac{E_{c}}{N_{0}+C_{k} N_{J} / \rho}
$$

where $E_{c}=E_{b} / N$ is the energy per subcarrier. Hence

$$
P_{B P S K}=P_{B P S K}\left(E_{b}, \rho, \sum_{k=0}^{N-1} S I N R_{k}\right) \leq P_{B P S K}\left(E_{b}, \rho, S I R\right)
$$

with equality when all subcarriers are uniformly interfered. Hence the receiver can use interference side information to improve the performance for Multi-tone interference as well.

\subsection{Conclusions}

The BER performance of different Interference strategies for OFDM system has been investigated. Both analytical forms and simulation values are given. In addition, two new Interference Injecting methods - optimal-fraction PBI and optimal-fraction MTI are proposed in this chapter. Through analysis and simulation, it is shown that under the various channel conditions, the optimal-fraction MTI clearly outperforms other interference strategies in the chapter. The results of the experiment and the analysis of those results show that the optimal-fraction MTI is a very effective interference injecting technique for OFDM system in various channel conditions. 


\section{CHAPTER V}

\section{ANTI-INTERFERENCE IN MC-CDMA SYSTEMS}

In this chapter, the anti-interference in MC-CDMA systems is considered. We only explore the single-user case. In the future, the analysis can be extended to multiple user case.

\subsection{Maximum likelihood Estimation of Interference Side Information}

In the proposed system, the received signal has constant envelope in both the time and frequency domain. This property can be used to generate the interference side information. In general, the interference will not cover the whole frequency domain and time domain simultaneously. Hence our anti-interference policy is listed as follows:

- Find the pulse interference in time domain.

- Get rid of pulse interference by time domain clipping

- Find the partial band interference

- Get rid of partial band interference by frequency domain clipping

In this policy, the key issue is finding correct interference side information in both the time domain and frequency domain. First, let us solve time-domain pulse interference mitigation problem. Given the transmitting symbol $u_{k}$ and the received symbol $y_{k}$, the amplitude difference $\| y_{k}|-| u_{k}||$ can be regarded as a Rayleigh distribution approximately. Due to the constant envelope property, all $\left|u_{k}\right|$ has the same value, which 
can be substituted with the mean value $M_{a}$. The following algorithm is exploited to get the variance $\sigma_{m}$ of amplitude difference.

1. $x \_n u m b e r=20$

2. Make all the time stubs active

3. Get $M_{a}$, the amplitude mean value of all active time stubs.

4. Based on $M_{a}$, calculate the amplitude variance of all the time stubs.

5. Deactivate $x \_n u m b e r$ time subs with biggest amplitude variance of all the time stubs.

6. $\mathrm{x} \_$number $=\mathrm{x} \_$number +20

7. Repeat 3-6 with the left subcarriers until 80 time stubs have been wiped out.

8. Get the mean value of amplitude difference, say, $M_{\text {diff }}=$ mean $\left(\left\|y_{k}|-| u_{k}\right\|\right)$ for all the active $k$

9. Get the amplitude difference variance of all active time stubs, that is,

$$
\sigma_{m}=\operatorname{var}\left(\| y_{k}|-| u_{k}||\right) \text { for all the active } k
$$

So

$$
\sigma_{R}^{2}=\frac{2}{4-\pi} \sigma_{m}^{2}
$$

Now what we should do is to measure the probability of $\hat{C}_{k}$. The log-likelihood estimate of $C_{k}$ is defined as

$$
L\left(\hat{C}_{k}\right)=\log \frac{\operatorname{Pr}\left(\hat{C}_{k}=0\right)}{\operatorname{Pr}\left(\hat{C}_{k}=1\right)}=\log \frac{1-\operatorname{Pr}\left(\hat{C}_{k}=1\right)}{\operatorname{Pr}\left(\hat{C}_{k}=1\right)}
$$

The probability of $\hat{C}_{k}=1$ is

$$
\operatorname{Pr}\left(\hat{C}_{k}=1\right)=\int_{L_{\text {down }}}^{L_{\text {up }}} p(y) d y
$$


where $p(y)$ is a Rayleigh probability density function (PDF) with mean $\sigma_{R} \sqrt{\frac{\pi}{2}}$, and variance $\frac{4-\pi}{2} \sigma_{R}^{2}$, and the $L_{u p}$ and $L_{d o w n}$ are given as follows

$$
\begin{gathered}
L_{u p}=|| y_{k}\left|-M_{a}\right| \\
L_{\text {down }}=0
\end{gathered}
$$

It is very clear that $L\left(\hat{C}_{k}\right)$ has been obtained for every sub-carrier. Obviously when $L\left(\hat{C}_{k}\right)<0$, the interference is deemed to be present at subcarrier $k$; when $L\left(\hat{C}_{k}\right)>0$, we decide that there is no interference at that sub-carrier. For every interfered subcarrier, we perform a nonlinearity clipping

$$
y_{k}=M_{a} e^{j \arg \left(y_{k}\right)}
$$

After that, the pulse interference in time domain has been eliminated.

This part describes the mitigation of partial band interference. Basically the same process can be applied except for the following consideration. Before the proceeding of interference mitigation, the zero forcing equalization will be done first. This operation will amplify the noise at the subcarriers where the channel response has smaller magnitude and mitigate the noise at the subcarriers where the channel response has a bigger magnitude. Consequently the noise variance for different subcarrier will not be the same any more. Hence, instead of using a single value $\sigma_{m}$, the extra operation is given as

$$
\sigma_{j, k}^{2}=\sigma_{m}^{2} /\left|\mathrm{H}_{\mathrm{d}, \mathrm{k}}\right|^{2} \quad 0 \leq k \leq N-1
$$

Similarly, the $\sigma_{R, k}^{2}$ is given by 


$$
\sigma_{R, k}^{2}=\frac{2}{4-\pi} \sigma_{j, k}^{2}
$$

All other operations are the same with the pulse noise elimination process. We omit the details for the sake of concision.

\subsection{Simulation Results}

In this part, the interference elimination policy is added to the downlink MC-CDMA receiver of Chapter 3 and simulated by Matlab. The 3GPP deployment TR 25.943 typical urban channel model is used as our test channel model. The system test parameters can be found in Table 3.1 .

There are three figures in total. Figure 5.1 gives us the simulation comparison results of the proposed system with/without interference mitigation policy under the pulse interference only. Figure 5.2 shows the same comparison except that the pulse interference changes to partial band interference. The effect of the pulse interference and partial band interference combination is presented in Figure 5.3. The interference parameter table is given as Table 5.1

The results are very apparent. The system with anti-interference part outperforms the one without anti-interference part. Due to the inter-impact of pulse interference and partial band interference, the anti-interference effect in Figure 5.3 is not as obvious as the one in Figure 5.1 and Figure 5.2.

Table 5.1. Interference parameters used in simulations

\begin{tabular}{|c|c|c|c|}
\hline Pulse interference SIR & $-2 \mathrm{db}$ & Partial band interference SIR & $-2 \mathrm{db}$ \\
\hline Pulse interference duty cycle & 0.25 & Partial band interference fraction & 0.25 \\
\hline
\end{tabular}




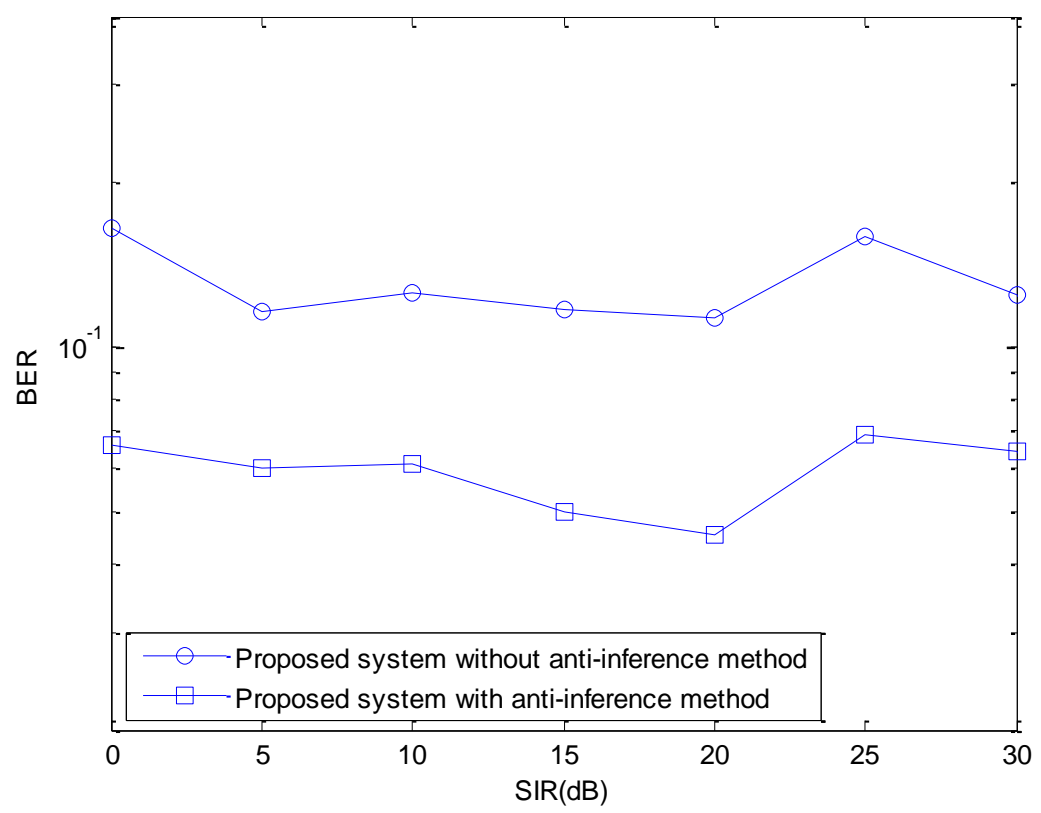

Figure 5.1. Comparison results of the proposed system with/without interference mitigation policy under the pulse interference

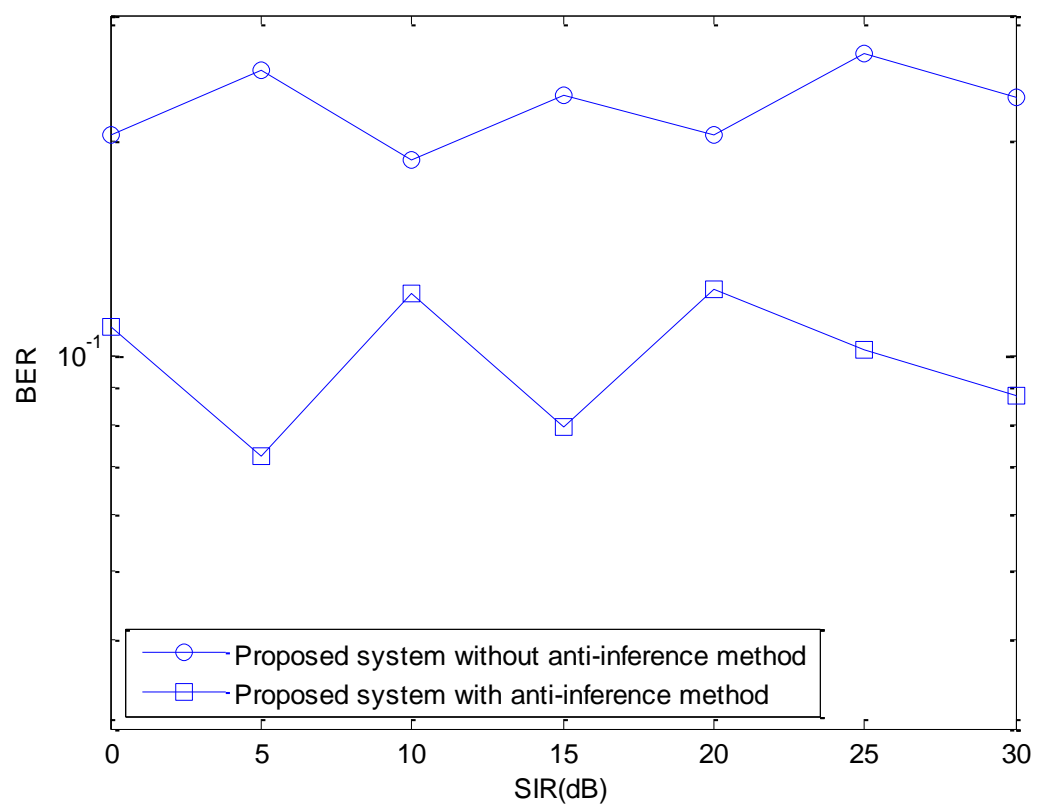

Figure 5.2. Comparison results of the proposed system with/without interference mitigation policy under the partial band interference 


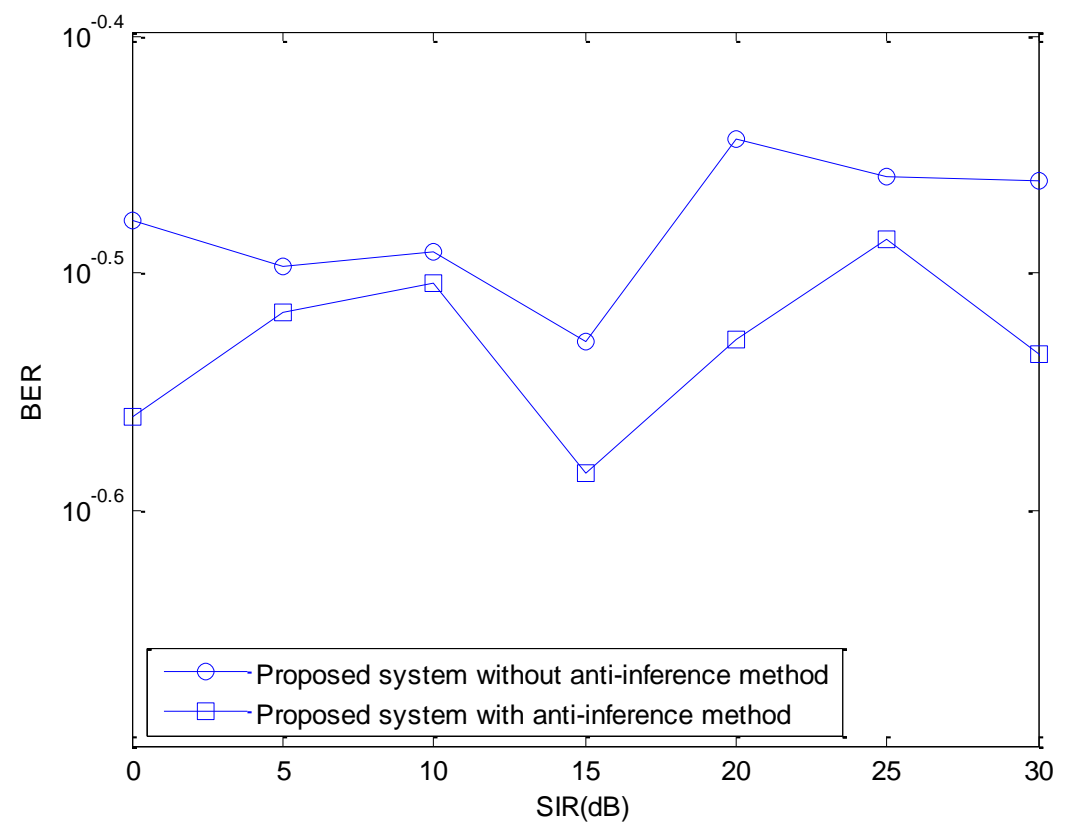

Figure 5.3. Comparison results of the proposed system with/without interference mitigation policy under the pulse interference and partial band interference

\subsection{Conclusions}

This chapter gives us a very simple but effective interference mitigation solution for MCCDMA systems .Both the analysis and simulation results show that the proposed method can reduce the pulse interference and partial band interference at the same time with low complexity. 


\section{CHAPTER VI}

\section{A PRACTICAL MC-CDMA SYSTEM ON SDR PLATFORM}

In this chapter, we give the reader a brief introduction of a practical MC-CDMA system on the software defined radio (SDR) platform.

\subsection{Software Defined Radio}

A software defined radio (SDR) is a radio that includes a transmitter in which the operating parameters of the transmitter, including the frequency range, modulation type or maximum radiated or conducted output power can be altered by making a change in software without making any hardware modifications [56]. The basic idea of SDR is shown in Figure 6.1.

There are a lot of benefits in the world of software radio [57]:

- The ability to change waveform and function on the fly. One general-purpose device can be configured into whatever kind of radio you are in need of at the moment, i.e., the possibility of adaptively choosing an operating frequency and a mode best suited for prevailing conditions.

- Quick and easy upgrading with new and enhanced features. Upgrading the software can be done over the air.

- Cost-effective solutions. When new waveforms are required in a system, there is no need to purchase new hardware equipment, just upload the new software remotely and you have a fully functional system with completely new features. Also, the elimination of analog hardware and its cost, results in simplification of radio architectures and improved performance. 


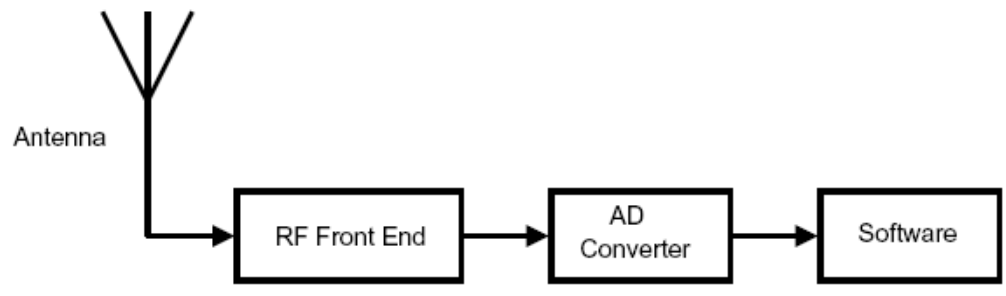

Receiving software radio

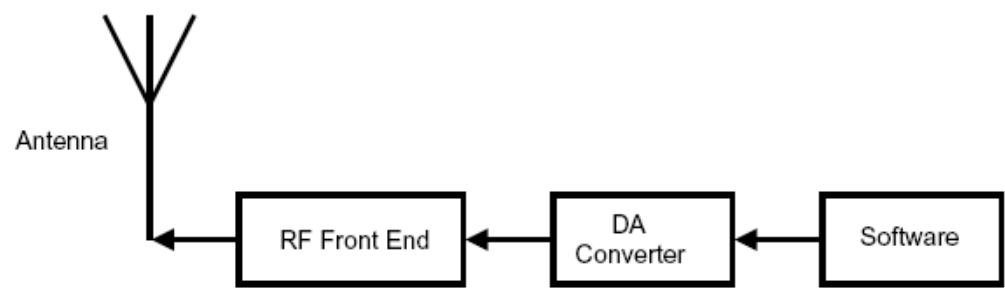

Transmitting software radio

Figure 6.1. Basics of SDR (from [57])

- Receive and broadcast multiple channels at the same time

- The opportunity to recognize and avoid interference with other communications channels

- Portability. An open standard architecture makes it possible for different kinds of communicators to talk to each other. It's not uncommon today that different types of government organizations such as police and fire departments from different areas can't communicate with each other due to the fact that they are using different types of radio systems. With SDR the communication devices can reconfigure themselves and thus "learn another language"

While SDRs offer benefits as outlined above, a few obstacles remain to their universal acceptance. Those include:

- The difficulty of writing software for various target systems.

- The need for interfaces to digital signals and algorithms. 
- Poor dynamic range in some SDR designs.

- A lack of understanding among designers as to what is required.

- There are some extra power consumption and complexity brought by the flexibility and compatibility.

- The SDRs are more vulnerable than the traditional radios.

\subsection{GNU radio}

GNU Radio is a collection of software that when combined with minimal hardware, allows the construction of radios where the actual waveforms transmitted and received are defined by software. What this means is that the digital modulation schemes used in today's high performance wireless devices are now software problems [58].

GNU Radio provides a library of signal processing blocks and the glue to tie it all together. The programmer builds a radio by creating a graph (as in graph theory) where the vertices are signal processing blocks and the edges represent the data flow between them. The signal processing blocks are implemented in C++. Conceptually, blocks process infinite streams of data flowing from their input ports to their output ports. Blocks' attributes include the number of input and output ports they have, as well as, the type of data that flows through each of them. Graphs are constructed and run in Python [59]. 


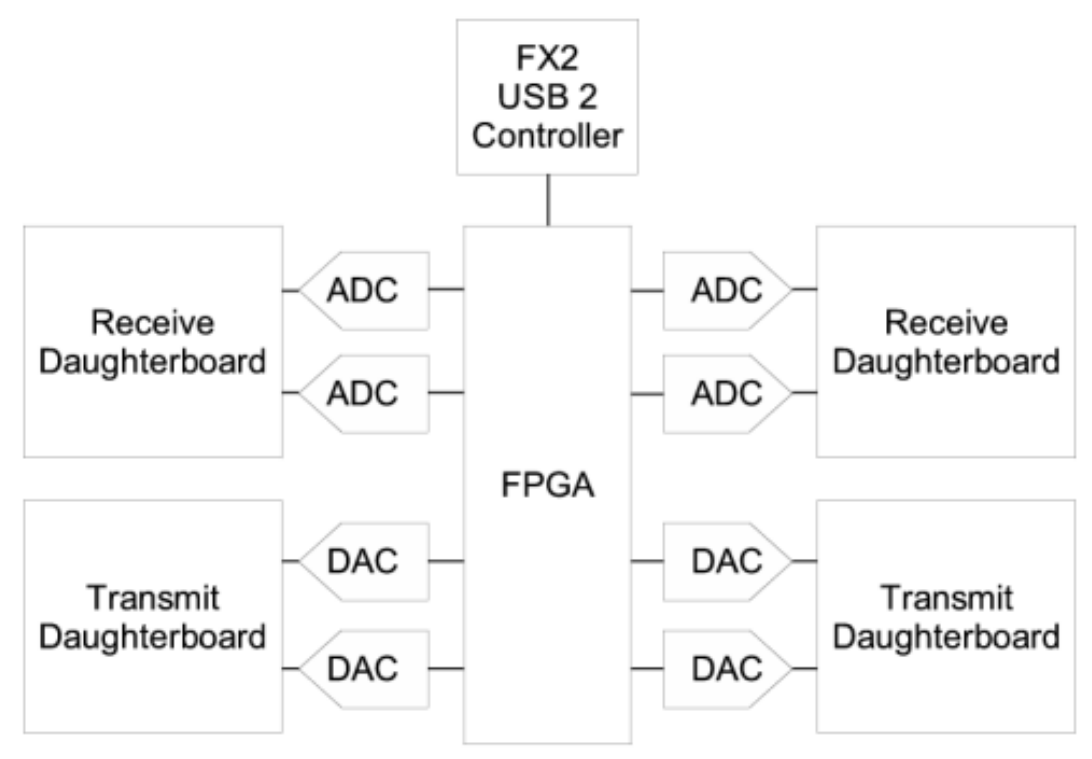

Figure 6.2. USRP (from 57)

GNU Radio is reasonably hardware-independent. Since it is pure free open source software, you can have different hardware solutions. Among them, the Universal Software Radio Peripheral (USRP) is the preferred one. Figure.6.2 shows the block diagram of the USRP. The brainchild of Matt Ettus, the USRP is an extremely flexible USB device that connects your PC to the RF world. The USRP consists of a small motherboard containing up to four 12-bit 64M sample/sec ADCs, four 14-bit, $128 \mathrm{M}$ sample/sec DACs, a million field programmable gate arrays (FPGA) and a programmable USB 2.0 controller. Each fully populated USRP motherboard supports four daughterboards, two for receive and two for transmit. RF front ends are implemented on the daughterboards. A variety of daughterboards is available to handle different frequency bands [59]. So, why should we use GNU radio? Its advantages are listed as follows [57]: 
- It is very easy to get started. With the help of processing block library and example grogram, it doesn't take more than a few minutes to have a small waveform application going.

- GNU radio is free open-source software. This makes it possible for anyone to expand the GNU Radio tool-kit with new functions and signal processing blocks.

- Good run-time system. The developer doesn't have to be concerned about data rates, memory allocation for communication buffers and so forth. One specifies what kind of data a block uses, and what data rate the inputs and outputs shall use. Then the run-time system does all the hard underlying work.

- Many tools and components are provided. Included in GNU Radio's core package are a vast number of ready-to-use components such as filters, signal sources and modulators for the waveform developer to use. It also comes with a spectrum analyzer and an oscilloscope.

- Hardware-independent. There exists cheap development hardware. USRP is one of them.

As other software, GNU Radio has some drawbacks as well:

- Lack of documentation. There are some tutorials on the web. But as far as we know, there is no documentation on how the underlying run-time system works. Of course you can read the freely distributed source code, but that is not an easy task. Certainly, along with the popularity of GNU Radio, this has been changed somehow. You can ask questions and search for some useful information in the developer forum. 
- Environment limitations. $\mathrm{C}++$ and Python are the only developing languages you can use. Also GNU Radio is primarily developed for the Linux platform.

- The USB interface does not allow for full duplex communication links implementation. This interface also limits the bandwidth of the processed signals to16MHz.

\subsection{FIU SDR Platform Project}

The specific technical problems for FIU SDR platform include:

- Intelligent antenna design. It is easy to construct wide-band software driven antenna tuners - many such devices are readily available on the market for the lower frequencies. Based on this, it is necessary to investigate available wide band antenna technologies.

- Programmable RF modules design. In the proposed system, the self-designed hardware is only concerning about the bandwidth from $25 \mathrm{MHz} \sim 1 \mathrm{GHz}$. For the carrier frequency above $1 \mathrm{GHz}$, the commercial hardware products will be applied to support ISM and IEEE 802.11a.

- ADC/DAC design. Power consumption seems to be one of the critical problems facing the design of DAC and ADC chips for SDR applications. Current technologies deal with this issue by using two ADCs for I and Q channels. While $\mathrm{RF}$ and linear devices have improved rapidly in recent years with the introduction of GaAs, SiGe and other advanced processes, mixed-signal devices such as ADCs and DACs have not improved quite as fast. For ADCs and DACs alike, the requirements are focused on noise and distortion. While ADCs most often focus 
on SNR and spurious-free dynamic range (SFDR), DACs are often specified by noise spectral density (NSD), adjacent-channel leakage ratio (ACLR), or adjacent-channel.

- Digital signal processing modules design. In today's DSP devices, processing of real-time applications is a serious challenge. However, for FPGAs this real-time processing seems to be more or less achievable. Therefore, in current technologies, designers have opted to optimally integrate DSPs and FPGAs in their SDR architectures. Among all the techniques including DSP chip, FPGA, ASIC and pure software implementation, we should select proper one for different function module based on the comprehensive consideration on performance, speed, development cost, technique risk, flexibility and so on.

- New software architecture is necessary for the proposed SDR system. To keep the developing cost lower, the architecture should be simple, but flexible, efficient and scalable.

- Self-diagnosis function. The self-diagnosis function includes one software component supplying diagnosis/debug information output, diagnosis/test vector data generation, hardware failure detection etc.

- Hierarchical security management. The various security functions such as authenticity, integrity, confidentiality etc. need to be supported in SDR receivers. In order to obtain these security functions various cryptographic algorithms and protocols can be used. Since various radio standards use different set of cryptographic algorithms and protocols, these algorithms and protocols need to be changed while reconfiguring the receiver to new SDR standard. 


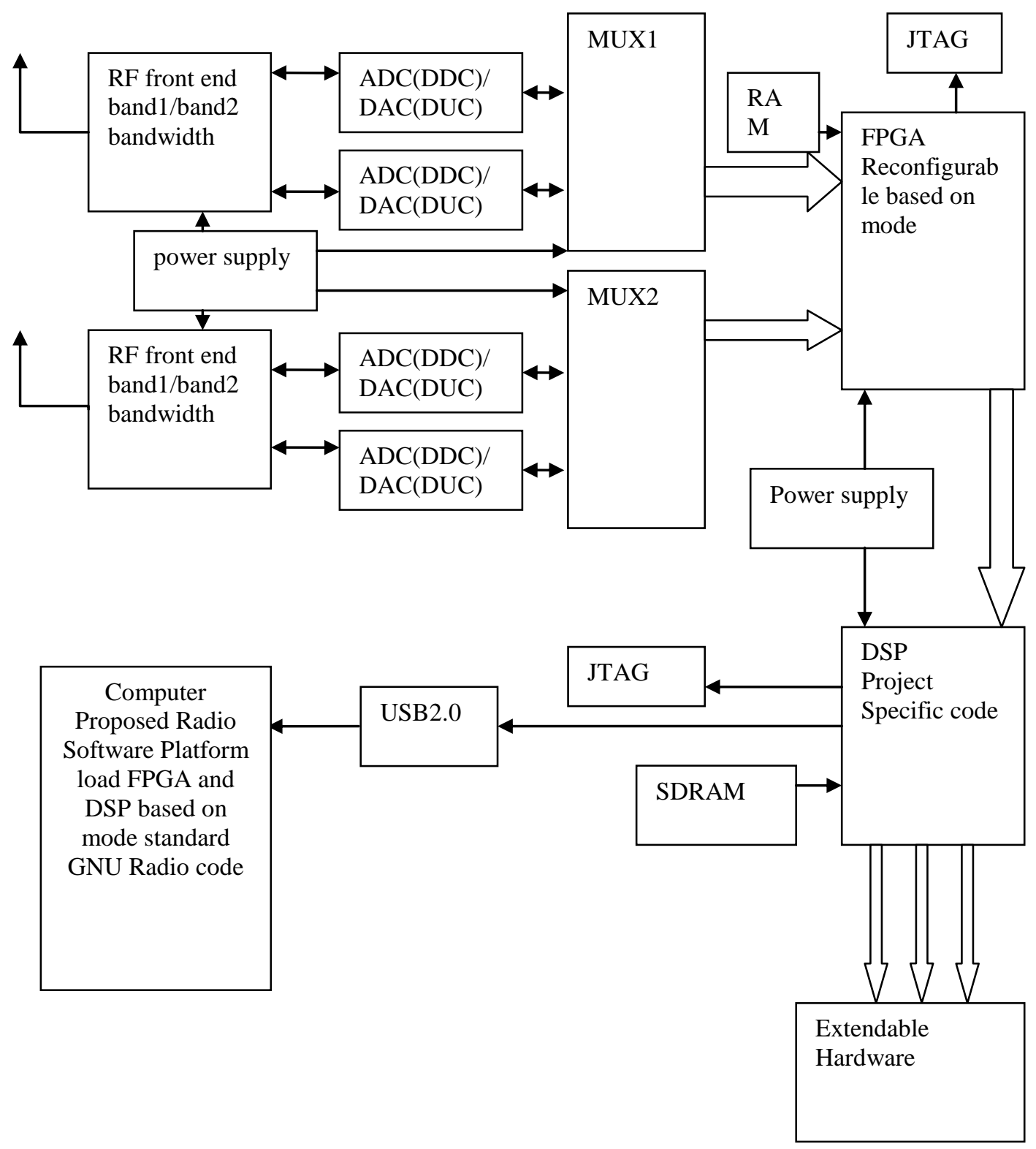

Figure 6.3. System Architecture

- Incremental developing method. We will design a general hardware structure based on small-scale applications, and then refine the components of the hardware based on different applications according to various requirements.

Basically, the system architecture is shown as Figure 6.3. 


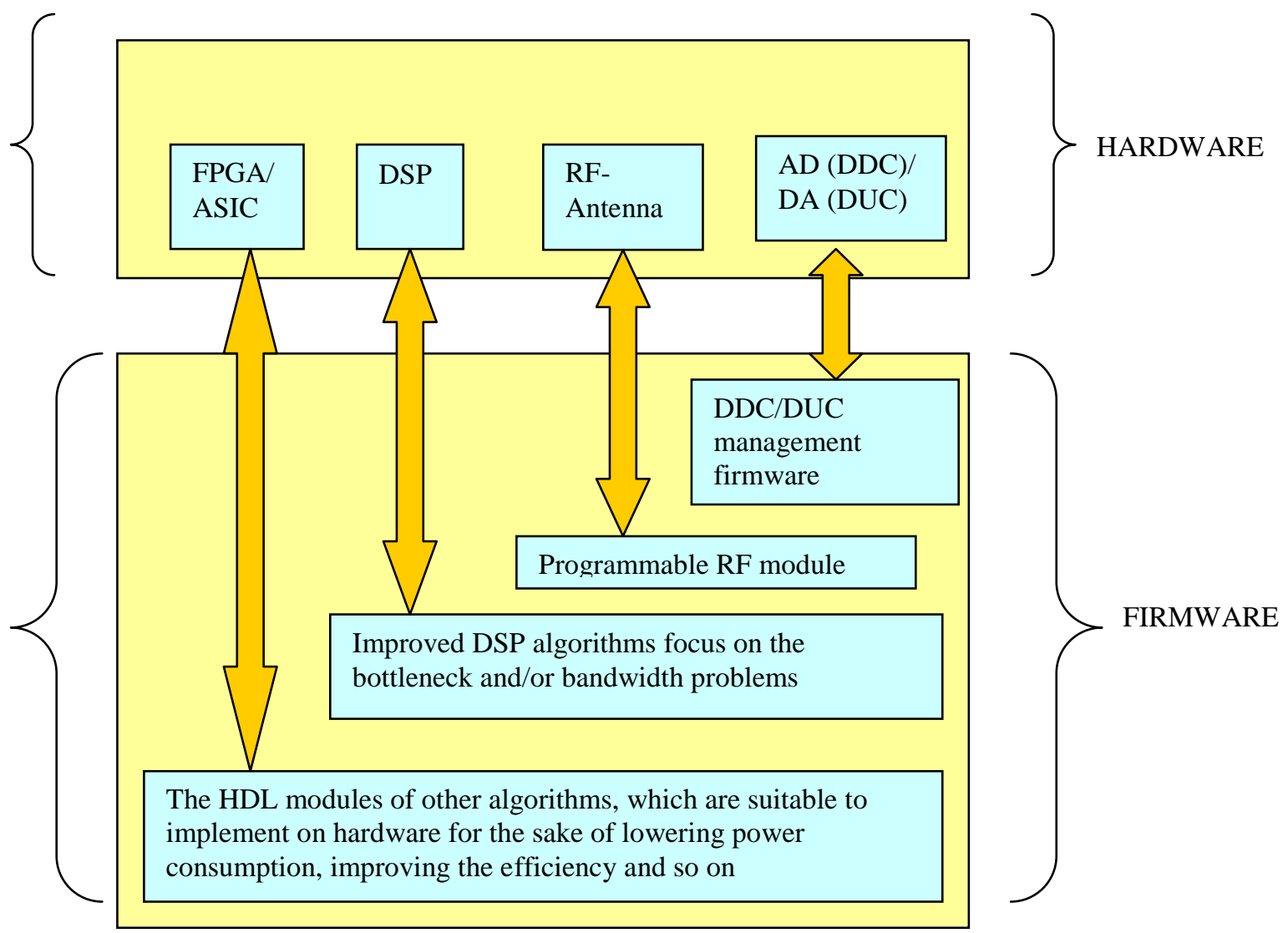

Figure 6.4. System Hardware Architecture

The system can be divided into 2 parts: One is the software part, located in the PC or equivalent embedded system, the other is the hardware part, which includes the actual hardware and the firmware running on it.

Figure 6.4 represents the hardware part. The corresponding parts can be found in Figure 6.3. It should be mentioned that the multiple number of antenna and RF frond is not mandatory. The hardware architecture only supports the carrier frequency from $25 \mathrm{MHz}$ to $1 \mathrm{GHz}$. For the carrier frequency above $1 \mathrm{GHz}$, the commercial product such as $802.11 \mathrm{a}$ wireless router will be employed. Since lowering the cost is very important in this project, our hardware design policy is to find a mature and low cost solution, which can satisfy our hardware requirements. General Purpose Processor (GPP) is available with adequate processing performance for many waveforms. However, the power utilization and heat 
dissipation of these devices is prohibitive in many size, weight, and power-limited systems. As a result, DSPs are typically required to supplement the GPP to meet modem architecture power budgets. Sometimes GPPs and DSPs employ a serial processing architecture that is not appropriate for many front-end preprocessing functions signal equalization, and radio frequency (RF) tuning. This problem is compounded when dealing with wideband waveforms, since the bandwidth of these waveforms may exceed the usable throughput of the serial processing architecture. The use of an FPGA-based processing element, which gives near application-specific integrated circuit (ASIC) performance in a programmable device, is thus often required.

The use of FPGAs and massive DSP is a costly solution compared with ASIC for a wide enough market. However, we will not consider developing ASICs due to the following reasons:

- ASIC means extremely slow time to market and great develop efforts. An ASIC development program involves at least 9-12 months per IC spin, and that typically 3-5 spins are needed before a commercially suitable chip set becomes available at economically viable quantities.

- $\quad$ ASIC has no any programmability, which is not suitable for the SDR system.

- For the small/middle size commercial applications, the cost of ASICs is larger than FPGAs.

- Although FPGAs and DSP consume more power, this problem becomes more and more trivial as the advanced techniques appear.

Therefore, we will use FPGA and DSP with additional off-the-shelf hardware to construct the proposed SDR hardware. 
The implementation of the software modules will be based in the GNU radio platform. GNU Radio is a collection of software that when combined with minimal hardware, allows the construction of radios where the actual waveforms transmitted and received are defined by software. What this means is that the digital modulation schemes used in today's high performance wireless devices are now software problems. It is free, open source and hardware independent. Rooted from the GNU Radio, the proposed software architecture will be developed.

The preferred hardware solution for the GNU radio system is the Universal Software Radio Peripheral (USRP). The USRP is an extremely flexible USB device that connects your PC to the RF world. The USRP consists of a small motherboard containing up to four 12-bit 64M sample/sec ADCs, four 14-bit, 128M sample/sec DACs, a million gatefield programmable gate array (FPGA) and a programmable USB 2.0 controller. Each fully populated USRP motherboard supports four daughterboards, two for receive and two for transmit. RF front ends are implemented on the daughterboards. A variety of daughterboards is available to handle different frequency bands. Low-power daughter boards are available that receive and transmit in the $440 \mathrm{MHz}$ and $1.24 \mathrm{GHz}$ amateur radio bands, targeted at the amateur radio applications. Licensing issues limit the use of these as actual transmitting prototypes except in overlapping government spectrum. A receive-only daughter board based on a cable modem tuner is available that covers the range from $50 \mathrm{MHz}$ to $800 \mathrm{MHz}$. Daughter boards are designed to be easy to prototype by hand in order to facilitate experimentation.

For amateur radio use, low-power daughterboards are available that receive and transmit in the $440 \mathrm{MHz}$ band and the $1.24 \mathrm{GHz}$ band. A receive-only daughterboard based on a 
cable modem tuner is available that covers the range from $50 \mathrm{MHz}$ to $800 \mathrm{MHz}$. Daughterboards are designed to be easy to prototype by hand in order to facilitate experimentation.

USRP is a very affordable choice. However, due to some of its limitations, we only use it in the Phase I. In this phase, the USRP based proof-of-concept will be developed, including the core system architecture and some application codes that implement 1 2 application scenarios. This proof-of-concept will demonstrate that the proposed system architecture design can be implemented in a practical system instead of a computer simulation. From the software point of view, the proof-of-concept will prove our novel software component design is effective in a real world situation. At the same time, from the view point of hardware, the proof-of-concept will show that existing low-scale, lowcost SDR hardware such as the USRP can support multi-band and multi-protocol communication systems.

Since the proof-of-concept has implemented part of the requirements with the specified hardware USRP, it is a non-optimal solution of our system, i.e., it realized the basic system functions in a non-optimal way. After intensive test and analysis, the system bottleneck and potential problems will be found. Aiming at those results, we will keep improving the system architecture in phase II. Specifically, since the USRP will be used as the hardware component for the Phase I proof-of-concept, at such stage the system will support a subset of the frequency bands required for Phase II. In Phase II, according to extra resource we get comparing to Phase I, we extend our prototype to a relatively largescale communication system. The core system architecture will still be based on the proof-of-concept; however, the system architecture should be improved to eliminate the 


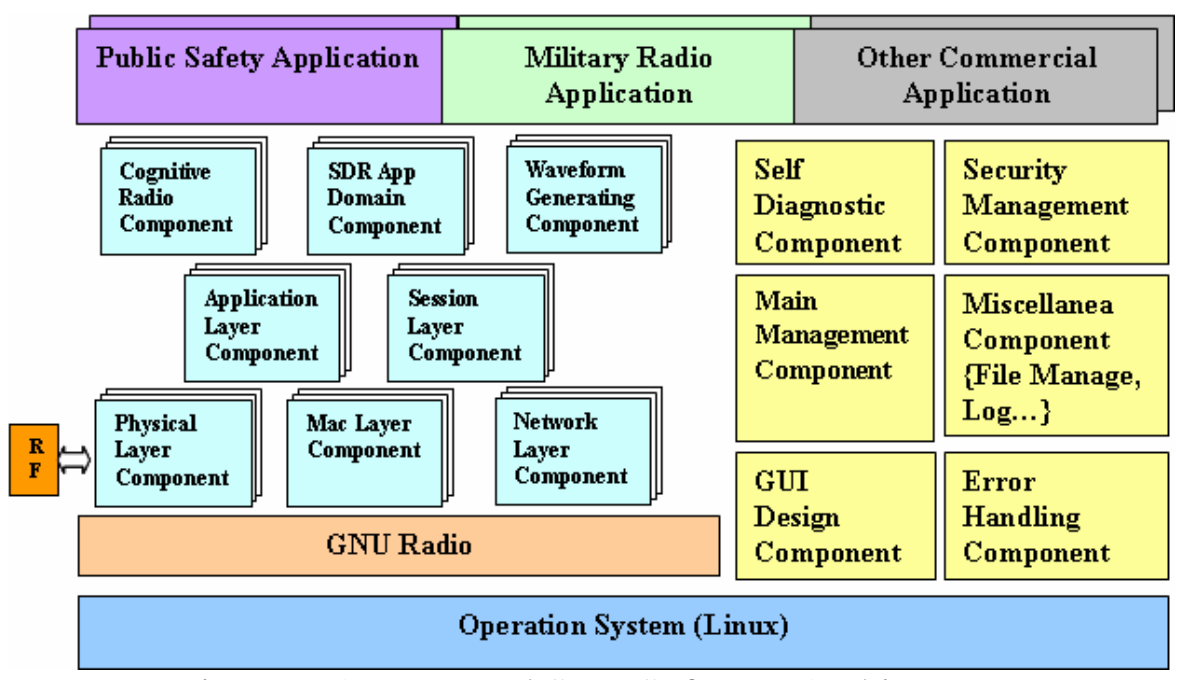

Figure 6.5. Proposed SDR Software Architecture

bottlenecks, solve the potential problems, and make the system more scalable and powerful. If necessary, more hardware parts such as FPGA and DSP will be added to the system. System characteristics such as throughput, interference cancellation, fast and accurate synchronization, efficient source coding, and various user oriented applications will be improved. The final system will have optimal performance, smaller size, lower power consumption etc.

As mentioned previously, the software architecture is a crucial part of the SDR system. In the Figure 6.5, the proposed software architecture for the Software Defined Radio system is displayed. As it can be seen, most of the required software modules will be developed within the GNU radio project.

The core concept of SDR is to solve the traditional radio transmitting and receiving problem in a software way, especially when a software implementation significantly enhances the performance of the resulting radio. Hence the bottleneck and the limitation factors appear more on the software side. Consequently, good software architecture is 
necessary to satisfy the system design requirement. The success of the concept of SDR depends on the historical performance improvements in digital technology which tends to follow Moore's Law. Moore's Law describes the rapid historically observed performance/cost improvements in digital IC capabilities. We note that analog technology, IC or discrete, does not follow Moore's Law because RFICs cannot take the same advantage of mask shrinks as is done with digital ICs. Therefore, replacing as much possible of the analog portion of a radio will eventually tend towards better performing and ultimately more capable and cheaper solution. It does, however, suffer from the massively increased requirement of highly sophisticated software development.

For the existing SCA architecture, there are still some unresolved issues. They want to do too much in it, and forget that it should be used in real time environment. For instance, the POSIX has been involved in the system to deal with Operation System compliance. This results in low performance efficiency obviously. Also CORBA is the core element in SCA architecture. In CORBA, all kinds of IDL, proxy processes consume a lot memory and processor speed, and only give us limited benefits.

Hence, we will give up some benefits (the interoperability of different SDR systems, the portability of applications software between different SDR implementations etc) brought by SCA since all the benefits are accompanied with large amount developing efforts. Instead, our goal is to achieve a simple software architecture design. We will not support different operation system (POSIX benefit). Only Linux will be used. We plan to use GNU radio as our base library to short developing time because GNU radio provides a 
library of signal processing blocks and the glue to tie it all together. That makes the developing efforts lower greatly.

Basically, software design is based on the function division principle. The functionoriented pipelining will be exploited. That is, functional transformations process their inputs to produce outputs. The programmer builds a radio by connecting different function components together. Different software components are defined by the function they perform in the system. The explanation of our proposed structure is given in Figure 6.5. Our design is object oriented and hierarchical based. Based on the network 7-layer definition, we create the corresponding components. In addition, in one component, there are some elegant design patterns. For example, we have an abstract base parent class for Network Layer component, which extracts and summarizes the common features for the network layer. The TCP/IC subclass is derived from it, supplying basic TCP/IC network support. All those components communicate and cooperate with each other, completing a specific function. For instance, the user should be able to change from the normal work mode to diagnosis mode. This includes the GUI component design, Diagnosis component design, and the diagnosis support from all other components.

Furthermore, the applications should be categorized; i.e., the applications share part of the application flow according to their similarities. This will be done in the Application Structure Component.

It is noticeable that there is one component called Waveform Generating Component. Different types of waveforms can be generated with this component. 


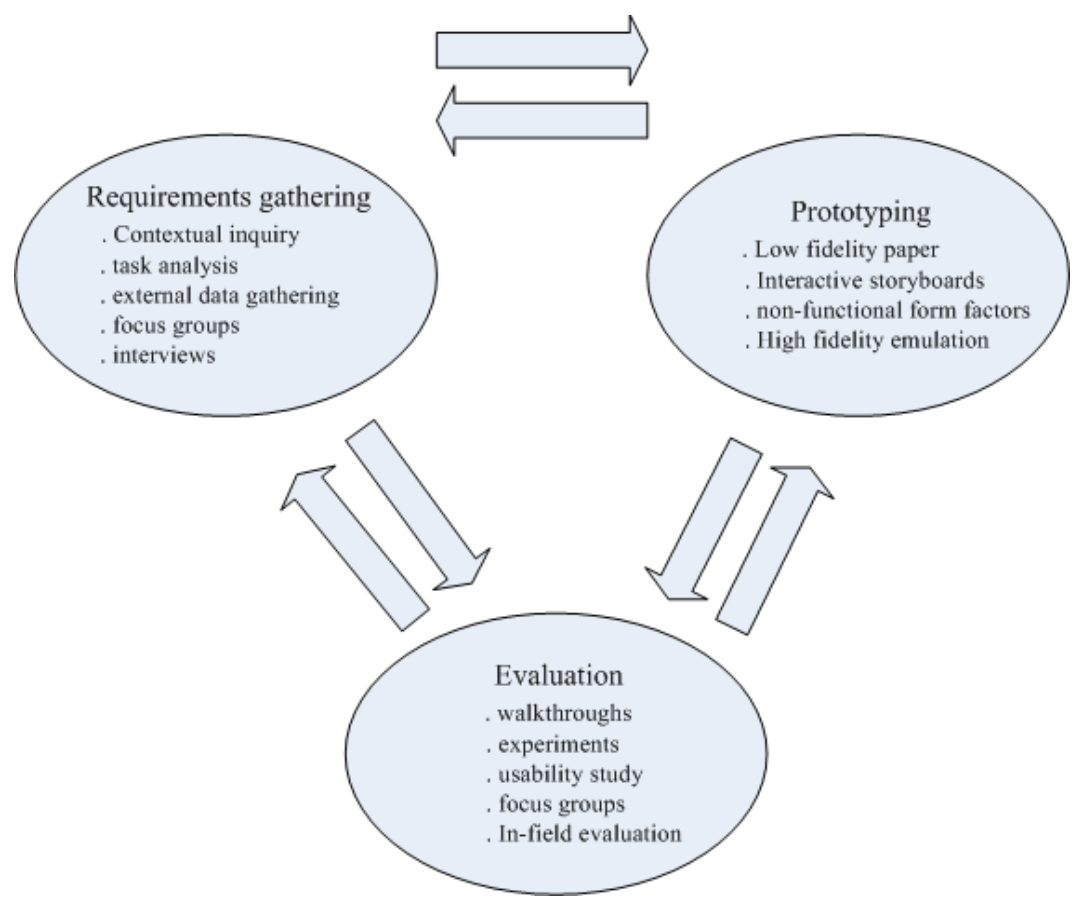

Figure 6.6. User-centered Design Process

The waveform generators that belong to one category such as Ground-to-ground waveform will have one common parent class, sharing some common properties and behaviors. Every waveform generator also has its own properties and behaviors. Through calling the diverse function components located in various layer of 7-layer structure, the waveform can be easily generated by the corresponding waveform generator. By this method similar to kids' building blocks, the new waveform generator may be added quite easily.

The hierarchical software obviously decreases the system efficiency. However, with this light-weight design, the requirement of most applications can be satisfied. Moreover, the user can choose to only use part of our software architecture, or even do not use it to maximize the performance. Therefore, in general, our software architecture supplies a powerful, scalable, and flexible solution. 
In the diagram shown in Figure 6.6, the user-centered design process to be used in the SDR system development is presented.

\subsection{A Cognitive Radio MC-CDMA System on FIU SDR platform}

Traditionally, radios have always been hardware based. They are manufactured to transmit and receive on a single frequency which is "hard-wired" at the time of manufacture. The use of the radio frequency (RF) spectrum by these devices is licensed by government regulatory bodies, such as the Federal Communication Commission (FCC) in the United States and the Australia Communications and Media Authority (ACMA) in Australia.

In 2004, the FCC proposed to allow unlicensed wireless devices to utilize vacant television channel frequencies in each market, a rulemaking that is currently in its final stages. The FCC discussed three methods (control signals, position determination, and cognitive radio with dynamic frequency selection) to ensure that unlicensed TV band devices operate only on vacant channels without harmful interference to broadcast TV service. Of these methods, cognitive radio has spurred the most debate. The cognitive radio method uses spectrum sensing and dynamic frequency selection (DFS) to identify and avoid occupied TV channels. This method has been approved by the Defense Department for unlicensed devices to share spectrum with military radar in the upper 5 $\mathrm{GHz}$ band. Potential service providers and equipment manufacturers embrace it because it does not require external infrastructure. However, TV broadcasters oppose it because they do not understand it and fear it will result in harmful interference. 


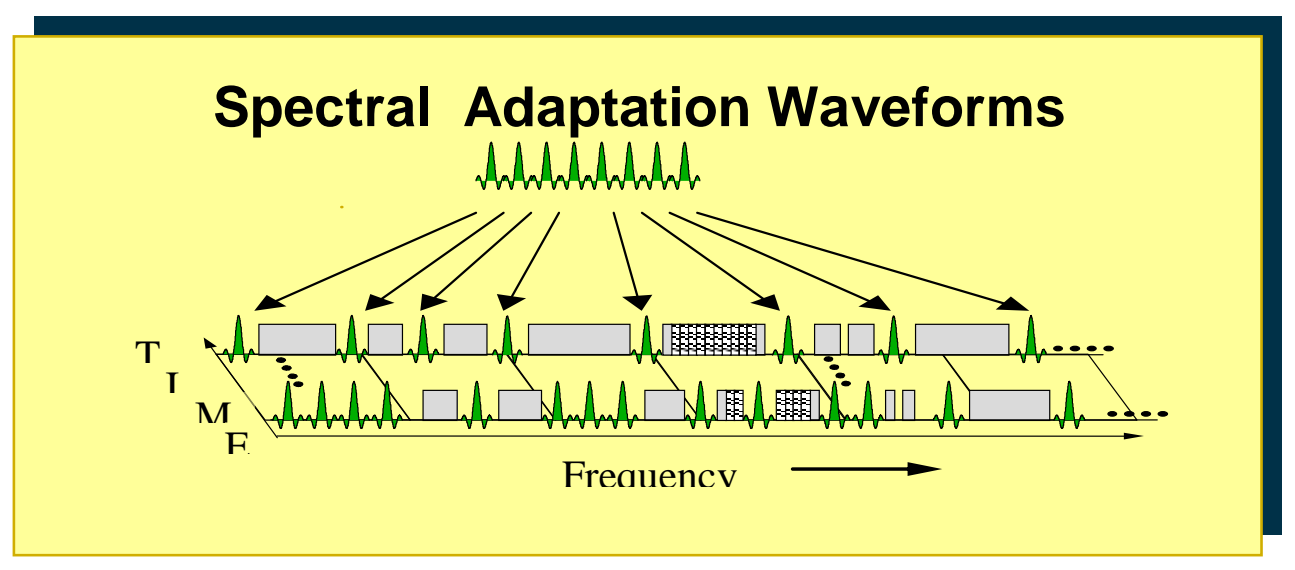

Figure 6.7. Spectral Adaptation Waveform

From Feb. 2009, along with the phase out of NTSC, the ability of detect NTSC signal is moot. The article gives us a clear picture: it is obvious that we can detect the HDTV signal easily even in most tough environment [63]. However we should consider the case of many cognitive radio networks coexisting case.

\subsubsection{Application Scenario}

As shown in Figure 6.7, TV channels are separated by unused frequencies. This "white space" in the broadcast spectrum varies with the channels present in a locale and creates opportunities for other applications. Hence our goal is to enable the deployment of wireless regional area networks using the unused TV channels, while not interfering with the licensed services now operating in the TV bands. 


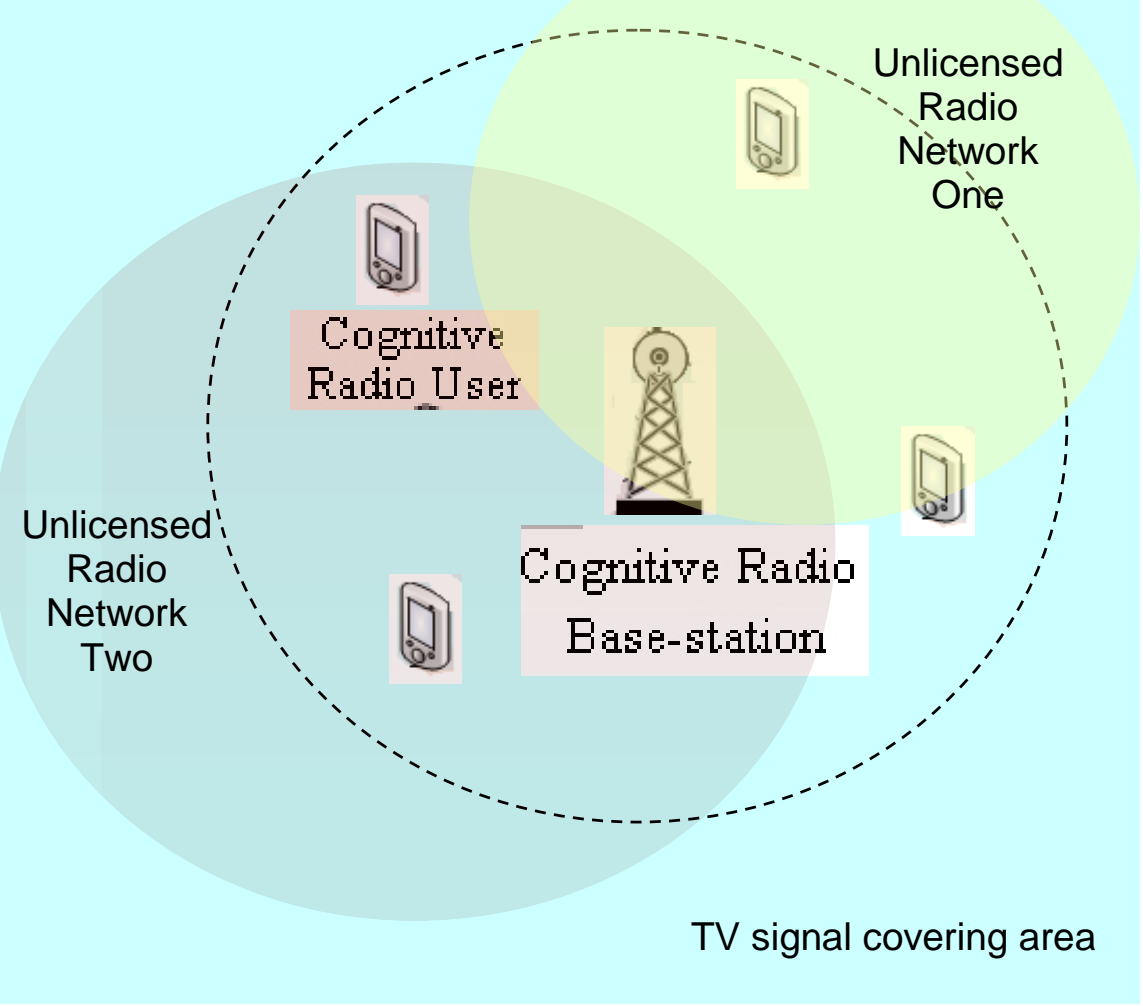

Figure 6.8. Cognitive Radio Network

In Figure 6.8, for cognitve radio mobile terminals and the BS, they should detect the occupied HDTV channel and the signal of other potential unlicensed cognitive radio users. Periodically, the cognitive BS and terminal will do the environment sensing. BS will gather all the information, then determine the system parameters, including the frequency band will be used, modulation scheme, power control method, coding rate and so on. Those parameters will keep unchanged until next sensing action begins. Since both BS and terminals know the previous Spectrum Sensing results, only changing parts are transmitted (Graded as three-bit number. For example, 111: occupied, 110: available, noise level: 9. 101: avaiable, noise level: 8 ..) If the environment is changing slowly, 
this means greatly decreased signaling. The system control parameters will be transmitted in the same way.

The proposed MC-CDMA system can be used in this case. However, different from the previous system structure, a new multi-group MC-CDMA scheme is exploited. For all available spectrum resource, we divide them into different quality groups. According to different transmission requirement such as QoS, data rate, BER etc, different users will be allocated into different groups with different system parameters. In single group, the data transmission is like the previous one in this paper.

\subsection{Experimental Results}

In the following demonstration, a picture in bitmap (bmp) format was transmitted using the GNU radio and USRP system. The information was transmitted through the wireless channel in packet form and using cyclic redundancy check (CRC) to detect any distortion to the transmitted data.

During the transmission of the image, the direction of the transmitting and receiving antennas was altered in order to induce some packets loss. The parameters used for this demonstration are shown in the following tables:

Table 6.1 Tx and Rx Parameters

For the transmitter side:

\begin{tabular}{|l|l|}
\hline Parameter & Value \\
\hline Bits per symbol & 1 \\
\hline Gray Code & True \\
\hline $\begin{array}{l}\text { RRC (Root-Raised } \\
\text { Cosine) roll-off factor }\end{array}$ & 0.35 \\
\hline Tx Amplitude & 12000 \\
\hline Modulation & DBPSK \\
\hline Bit rate & $50 \mathrm{kbps}$ \\
\hline Samples per symbol & 5 \\
\hline Interpolation & 512 \\
\hline
\end{tabular}

For the Receiver Side:

\begin{tabular}{|l|l|} 
Parameter & Value \\
\hline Bits per symbol & 1 \\
\hline Gray Code & True \\
\hline $\begin{array}{l}\text { RRC (Root-Raised } \\
\text { Cosine) roll-off factor }\end{array}$ & 0.35 \\
\hline Rx Gain & 45 \\
\hline Modulation & DBPSK \\
\hline Bit rate & $50 \mathrm{kbps}$ \\
\hline Samples per symbol & 5 \\
\hline Decimation & 256 \\
\hline
\end{tabular}




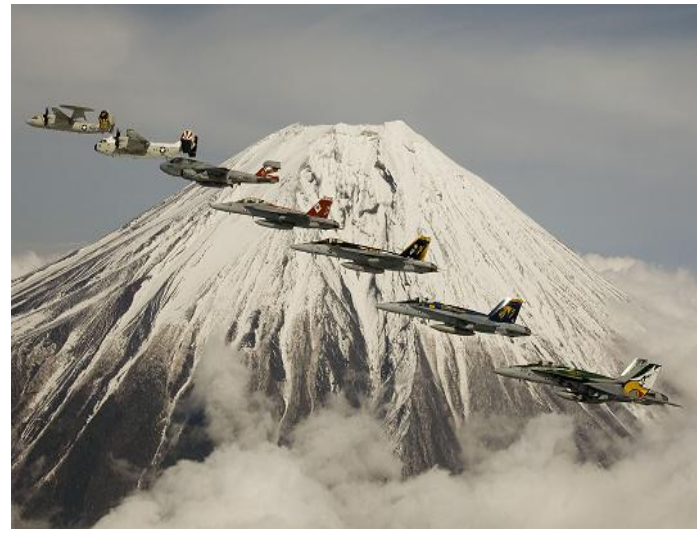

Figure 6.9 Transmitted bmp Image

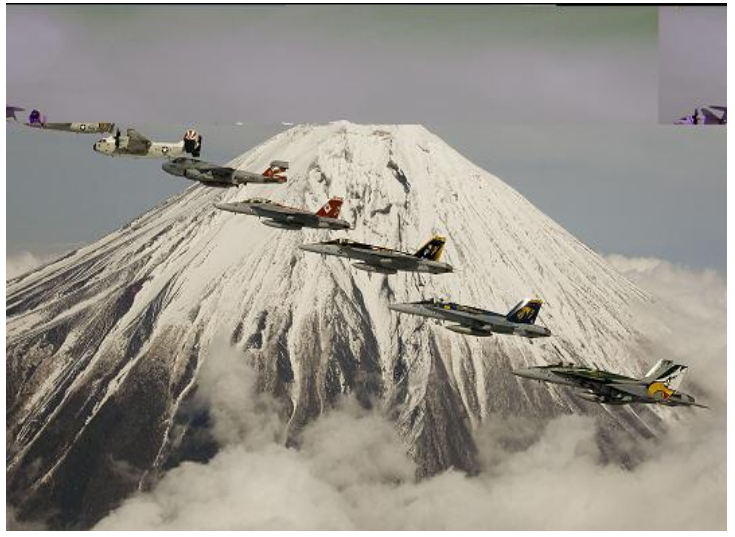

Fig. 6.10 Received bmp Image

Notice how due to the intentional change in direction of the $\mathrm{Tx}$ and $\mathrm{Rx}$ antennas the resulting image is smaller and distorted, shown as a shift of pixels at the top of the image. This shift, as previously mentioned, is caused by the intended loss of some packets during transmission. 


\section{CHAPTER VII}

\section{CONCLUSIONS}

This research presents a complete solution for MC-CDMA systems. We try to solve MAI and PAPR problem in a low complexity way. In the first section, we give what we have done in this thesis. In the second section, we talk about the future work.

\subsection{Summary of Current Work}

In this dissertation, we design a novel MC-CDMA system, which has low PAPR and mitigated MAI. We solve the problem from the root, i.e., we use CQS instead of Walsh codes because it reduced the PAPR greatly and mitigate MAI effectively as well. The new semi-blind channel estimation and multi-user data detection based on Parallel Interference Cancellation (PIC) have been applied in the system. The Low Density Parity Codes (LDPC) has also been introduced into the system to improve the system performance. Different interference models are analyzed in multi-carrier communication system and then the effective interference suppression for MC-CDMA system is employed in this dissertation. The experimental results indicate that our system not only significantly reduces the PAPR and MAI but also effectively suppresses the outside interference with low complexity. The practical system based on software defined radio platform - GNU Radio is on the way of developing. The potential applications include JTRS military SDR system and HD-TV band cognitive radio system. 


\subsection{Future Work}

There are several topics that deserve to be future studied.

- Uplink channel estimation and data detection:

In this thesis, for channel estimation and data detection part, we focus on the downlink part. In the downlink MC-CDMA system, the decoder is not involved in the PIC cancellation process as shown in [22] and [23] since it is not feasible to implement this kind of processing on a MT due to its complexity. In the uplink MCCDMA system, besides the exploiting of pre-equalization, the iterative receiver with the feedback extrinsic probability from the decoder can be applied to obtain better performance.

- Interference mitigation

The interference mitigation in this work has been proved simple and effective. However, the interference mitigation method mentioned in this thesis is only for single user case. In the future, the interference mitigation method should be extended to multiple user case. If the interference has a predictable structure, it is possible to subtract the predicted interference signal from the received signal. Adaptive filtering techniques can be used to minimize the error involved in such a predictive interference signal. The subspace processing techniques can perform interference excision as well. Most computational techniques are useful only for narrowband interference; while antenna based techniques prove useful against wideband interference. The choice of anti-interference technique depends on the environment, cost, size etc. A combination of technique might be necessary to combat interference. 
- SDR and Cognitive Radio

This part is far away from being mature. With roots in today's SDR technology, cognitive radios offer the next level of software radio functionality. As regulatory hurdles diminish, such radios are moving closer to reality. This paper gives us a clear picture: how to apply our research results to a practical SDR cognitive radio system. Although there are many unsolved problems, those techniques will have a bright future. 


\section{REFERENCES}

1. K. Fazel, S. Kaiser, Multi-Carrier and Spread Spectrum Systems, John Wiley \& Sons Ltd, New York, 2003

2. R. Dinis, P. Silva, "Analytical evaluation of nonlinear effects in MC-CDMA signals," IEEE Tran. on Wireless Comm., vol. 5, no. 8, pp 2277- 2284, Aug. 2006

3. D. J. C. MacKay and R. M. Neal, "Near Shannon limit performance of low density parity check codes," Electron. Lett., vol.32, no.18, pp.1645-1646, 1996.

4. L. Xiong, Z. Tan, Y. Wen, X. Chen, "On the performance of LDPC coded MCCDMA in Rayleigh fading channels,” IEEE MAPE, vol.2, pp.1178-1181, 2005.

5. Y. Lee, Y. Kang, M. Noh, and H. Park, "Effects of Subcarrier Interleaving on LDPC Coded MC-CDMA Systems," International Workshop on Multi-Carrier Spread Spectrum (MC-SS 2005), Oberpfaffenhofen, Germany, 2005.

6. A. Serener, B. Natarajan and D. Gruenbacher, "Performance of spread OFDM with LDPC coding in outdoor environments," Proceedings of IEEE 58th Semiannual Vehicular Technology Conference, Orlando, Florida, October 2003.

7. J. G. Andrews, "Interference cancellation for cellular systems: a contemporary overview,” IEEE Wireless Commun. Magazine, vol. 12, pp. 19-29, Apr. 2005.

8. N. Benvenuto and P. Bisaglia, "Parallel and Successive Interference Cancellation for MC-CDMA and Their Near-far Resistance," IEEE VTC, vol. 2, pp. 1045-1049, Oct. 2003.

9. M. Kobayashi, J. Boutros and G. Caire, "Successive Interference Cancellation with SISO Decoding and EM Channel Estimation," IEEE Journal on Selected Areas in Comm., vol. 19, pp. 1450--1460, Aug. 2001.

10. B. P. Hombs and J. S. Lehnert, "Multiple-access interference suppression for MCCDMA by frequency domain oversampling," IEEE Transactions on Communications, vol. 53, no. 4, pp. 677-686, April 2005.

11. D. Mottier and D. Castelain, "SINR-Based Channel Pre-equalization for Uplink Multi-Carrier CDMA Systems”, PIMRC 2002, pp. 1488-1492, Sept. 2002.

12. J. Tan, G. L. Stuber, "Multicarrier Spread Spectrum System with Constant Envelope: Anti-Jamming, Jamming Estimation, Multi-User Access," IEEE Transactions on Wireless Communications, vol. 4, no. 4, pp.1527-1538, 2005. 
13. Z. Shen, J. Andrews, and B. Evans, "Short range wireless channel prediction using local information," in The Thirty-Seventh Asilomar Conference on Signals, Systems and Computers, vol. 1, 9-12 Nov. 2003, pp. 1147-1151.

14. V. Erceg et al., "Channel Models for Fixed Wireless Applications," (IEEE802.16a03/01): Broadband Wireless Working Group, IEEE P802.16, 2003.

15. H. Futaki, T. Ohtsuki, "Low-Density parity-Check (LDPC) Coded OFDM Systems", IEEE VTC'01 Fall, vol. 1, pp 82--86, Atlantic, Oct. 2001.

16. J. Luo et al., "Performance of LDPC Coded MC-CDMA Downlink System with Constant Envelope Spread Sequences,” IEEE MILCOM Oct. 2007

17. G. B. Folland., Real Analysis: Modern Techniques and Their Applications, Second Edition. Wiley-Interscience, 1999.

18. IEEE 802.16, "Part 16: Air interface for fixed Broadband Wireless Access Systems," IEEE 802.16 Standard, June. 2004.

19. Q. Shi and M. Latva-aho, "An exact error floor for downlink MC-CDMA in correlated Rayleigh fading channels," IEEE Commun. Letter, vol.6, no.5, pp. 196198, May 2002.

20. D. Motter, "Spreading sequences for uplink and downlink MC-CDMA systems: PAPR and MAI minimization," IEEE Trans. Commun., vol.13, no.5, pp.465-471, Sept.-Oct. 2002.

21. M. Hsieh and C.Wei, "Channel estimation for OFDM systems based on comb-type pilot arrangement in frequency selective fading channels," IEEE Trans. Consumer Electron., vol. 44, no. 1, Feb. 1998.

22. X. Wang and H.V. Poor, "Iterative (Turbo) Soft Interference Cancellation and Decoding for Coded CDMA," IEEE Transactions on Communications, 46(7), pp.1046-1061, July 1999.

23. T. Zemen, C. F. Mecklenbr"auker, J. Wehinger, and R. R. M"uller, "Iterative joint time-variant channel estimation and multi-user decoding for MC-CDMA," IEEE Trans. Wireless Communications, vol. 5, no. 6, June 2006.

24. Jan-Jaap van de Beek, Ove Edfors, Magnus Sandell, Sarah Kate Wilson, and Per Ola BÄorjesson. On channel esimation in OFDM systems. In Proc. VTC'95, volume 2, pages 715-719, Rosemont, IL, July 1995. IEEE. 
25. O. Edfors, M. Sandell, J.-J. van de Beek, S. K. Wilson, and P. O. Brjesson, "OFDM channel estimation by singular value decomposition," IEEE Trans. Commun., vol. 46, no. 7, pp. 931-939, Jul. 1998.

26. Y. Gong, and K.B. Letaief, "Low rank channel estimation for space-time coded wideband OFDM systems," Proceedings of the 54th IEEE Vehicular Technology Conference, vol.2, pp. $772-776$, Oct. 2001.

27. Y. R. Zheng and C. Xiao, "Improved models for the generation of multiple uncorrelated Rayleigh fading waveforms," IEEE Commun. Letter, vol. 6, no. 6, pp. 256-258, June 2002.

28. H. Poor, An Introduction to Signal Detection and Estimation. New York: SpringerVerlag, 1985, ch. 4.

29. Baudais (J.-Y.), Helard (J.-F.), Citerne (J.), “An improved linear MMSE detection technique for Multi-Carrier CDMA systems: comparison and combination with interference cancellation," European Transactions on Telecommunications, Special issue on Multi-Carrier Spread-Spectrum, vol. 11, pp.547-554, (December 2000).

30. C. Patel, G. L. Stüber, T. Pratt, "Analysis of OFDM/MC-CDMA Under Imperfect Channel Estimation and Jamming," IEEE Wireless Communications Networking Conference, Atlanta, GA, March 2004.

31. 3GPP TSG RAN WG4, Deployment Aspects, 3G TR 25.943 V1.2.0, June 2001.

32. S. Nobilet and J. Helard, "A pre-equalization technique for uplink MC-CDMA systems using TDD and FDD modes," Proc. VTC 2002-Fall, vol.1, pp.346-350, Sept. 2002.

33. D. Mottier and D. Castelain, "SINR-based channel pre-equalization for uplink multicarrier CDMA systems," Proc. 13th IEEE International Symposium on Personal, Indoor and Mobile Radio Communications, vol.4, pp.1488-1492, Sept. 2002.

34. P. Bisaglia, N. Benvenuto, and S. Quitadamo, "Performance Comparison of SingleUser Pre-equalization Techniques for Uplink MC-CDMA Systems," GLOBECOM'03, pp. 3402-3406, Dec. 2003.

35. G. MKRTCHYAN, K. NAITO, K. MORI, H. KOBAYASHI, "Model Based Prediction of Uplink Multi-Path Fading Channel Response for Pre-Equalization in Mobile MC-CDMA Systems," IEICE TRANS. COMMUN., VOL.E91-B, NO.2 FEBRUARY 2008.

36. J. G. Andrews and T. Meng, "Optimum Power Control for Successive Interference Cancellation with Imperfect Channel Estimation," IEEE Trans. Wireless Commun., vol. 2, no. 2, pp. 375-383, Mar. 2003. 
37. M. Mozaffaripour, N. Neda, and R. Tafazolli, "Partial parallel interference cancellation and its modified adaptive implementation for MC-CDMA system," IEE 3G Mobile Communication Technologies, pp. 281- 285, 2004.

38. S. Kaiser and J. Hagenauer, "Multicarrier CDMA with Iterative Decoding and SoftInterference Cancellation,” Proc. IEEE GLOBECOM, pp. 6-10, Nov. 1997.

39. N. Benvenuto, P. Bisaglia, and M. Finco, "Soft-interference cancellation for downlink and uplink MCCDMA systems," in Proc. 15th IEEE International Symposium on Personal, Indoor and Mobile Radio Communications, vol. 1, pp. 170-174, Sept. 2004.

40. X. Wang and H. V. Poor, "Iterative (turbo) soft interference cancellation and decoding for coded CDMA,” IEEE Trans. Commun., vol. 47, no. 7, pp. 1046-1061, 1999.

41. S. Morosi, R. Fantacci, and A. Bernacchioni, "Improved Iterative Parallel Interference Cancellation Receiver for Future Wireless DS-CDMA Systems," EURASIP Journal on Applied Signal Processing, no.5, pp. 626-634, 2005.

42. Z. Shi and C. Schlegel., "Iterative Multiuser Detection and Error Control Code Decoding in Random CDMA," IEEE Trans. Signal Processing, vol. 54, no. 5, May 2006.

43. D. Divsalar, M. K. Simon, and D. Raphaeli, "Improved parallel interference cancellation for CDMA," IEEE Trans. Commun., vol.46, no.2, pp.258-268, Feb. 1998.

44. B. Sklar, Digital Communications - Fundamentals and Applications, pp. 494, 2nd Ed. Englewood Cliffs, NJ, Prentice-Hall PTR, 2001.

45. A. Goldsmith, Wireless Communications, Cambridge University Press, 2005.

46. R. F. Ormondroyd and E. Al-Susa, "Impact of multipath fading and partial-band interference on the performance of a COFDM/CDMA modulation scheme for robust wireless communications," IEEE MILCOM, vol. 2, pp. 673-678, 1998.

47. H. Zhang and Y. Li, "Anti-jamming property of clustered OFDM for dispersive channels," IEEE MILCOM, vol. 1, pp. 336-340, October 2003.

48. J. Park et al., "Effect of partial band jamming on OFDM-based WLAN in 802.11g," ICASSP 2003, vol. 4, pp. 560-563, April 2003. 
49. S. Lijun et al., "BER Performance of Frequency Domain Differential Demodulation OFDM in Flat Fading Channel," GLOBECOM, vol. 1, pp. 1-5, 2003.

50. N. Kostov, "Mobile Radio Channels Modeling in Matlab," Journal of RADIOENGINEERING, vol. 12, part 4, pp. 12-17, 2003.

51. A. Papoulis, Probability, Random Variables and Stochastic Processes, 3rd Edition, McGraw-Hill, New York, Feb. 1991

52. N. Kingsbury, "Approximation Formulae for the Gaussian Error Integral, Q(x)," Connexions, June 7, 2005.

53. L. Hanzo et al., OFDM and MC-CDMA for Broadband Multi-User Communications, WLANs and Broadcasting, Wiley-IEEE Press, September 2003.

54. IEEE 802.11a, "Part 11: Wireless LAN Medium Access Control (MAC) and Physical Layer (PHY) Specifications: High-speed Physical Layer in the $5 \mathrm{GHz}$ Band," supplement to IEEE 802.11 Standard, September 1999.

55. Y. R. Zheng and C. Xiao, "Improved models for the generation of multiple uncorrelated Rayleigh fading waveforms," IEEE Communications Letters, vol. 6, no. 6, pp. 256-258, 2002.

56. Jesper M. Kristensen, “GNU radio an introduction,” Mobile Developer Days 2007.

57. Thomas Sundquist, "Waveform Development using Software Defined Radio," University essay from Linköpings University 2006.

58. http://gnuradio.org/trac/wiki.

59. http://www.gnu.org/software/gnuradio/doc/exploring-gnuradio.html. A high level overview of GNU Radio and the USRP.

60. Eric Blossom, “A introduction to Software Radio,” USENIX, Boston, June 3, 2006.

61. Lee K. Patton, “A GNU Radio Based Software-Defined Radar," thesis, Wright State University, 2007.

62. Ketan Mandke, Robert C. Daniels and Soon-Hyeok Choi "A MIMO demonstration of Hydra," WINTECH, Montreal, QC, Canada, 2007.

63. Mark A. Sturza, Farzad Ghazvinian, "Can Cognitive Radio Operating in the TV White Spaces Completely Protect Licensed TV Broadcasting?" An Engineering Study, January 30, 2007 
VITA

\section{JUN LUO}

1992-1996

1996-1999

1999-2003

2003-2005

2005-2008
B.S., Electrical Engineering, Nanjing University, Nanjing, China.

The $28^{\text {th }}$ Institute, Nanjing, China

Hoperun Software Ltd., Nanjing, China

M.S., IC Design, Beihang University, Beijing, China

Doctoral Candidate, Electrical Engineering

Florida International University

Miami, FL

\section{PUBLICATIONS AND PRESENTATIONS}

\section{Book Chapter}

Jun Luo, Jean H. Andrian, Chi Zhou, and James P. Stephens, Sr., "Performance Analysis of Interference for OFDM Systems," in Wireless Technology: Applications, Management, and Security, Springer Book, 2008 (To appear)

Journal

Jun Luo, Omar Granados, Te-Shun Chou, Huijing Qiang, and, Jean H. Andrian "Semiblind channel estimation and multi-user data detection for downlink MC-CDMA Systems," submitted to IEEE transaction on Vehicular Technology. June, 2008

Te-Shun Chou, Kang K. Yen, Jun Luo, "Network Intrusion Detection Design Using Feature Selection of Soft Computing Paradigms," International Journal of Computational Intelligence, Volume 4, Number 3, pp. 205-217, 2007.

\section{Conference}

Jun Luo, Jean H. Andrian, Chi Zhou, and Te-Shun Chou "Soft Parallel Interference Cancellation for a Turbo Coded Uplink MC-CDMA System Bit Error Rate Analysis of Jamming for OFDM System," IEEE 7th annual Wireless Telecommunications Symposium (WTS 2008), Pomona, California, April 26-28, 2008.

Jun Luo, Jean H. Andrian, Chi Zhou, "Bit Error Rate Analysis of Jamming for OFDM System," IEEE 6th annual Wireless Telecommunications Symposium (WTS 2007), Pomona, California, April 26-28, 2007. 
Jun Luo, Jean H. Andrian, Chi Zhou, and James P. Stephens, Sr., "PERFORMANCE OF LDPC CODED MC-CDMA DOWNLINK SYSTEM WITH. CONSTANT ENVELOPE SPREAD SEQUENCESS," IEEE Milcom 2007, Orlando, Florida, Oct. 29-31 2007.

Te-Shun Chou, Kang K. Yen, Jun Luo, Niki Pissinou, and Kia Makki, "CorrelationBased Feature Selection for Intrusion Detection Design," IEEE Milcom 2007, Orlando, Florida, Oct. 29-31 2007.

Te-Shun Chou, Kang K. Yen, and Jun Luo, "Feature Reduction and Fuzzy Belief Intrusion Detection Design," The 11th World Multi-Conference on Systemics, Cybernetics and Informatics jointly with The 13th International Conference on Information Systems Analysis and Synthesis, pp. 262-267, Orlando, FL, July, 2007.

Other Academic Activities

Student Member, Institute of Electrical and Electronics Engineers (IEEE)

Reviewer, IEEE International Conference on Communications 2008

Reviewer, Springer Book, Wireless Technology: Applications, Management, and Security

FIU Logic Lab 3712L instructor

Co-author, the Software Defined Radio bidding proposal for VIPMobile Inc. (Funded).

Co-author, the Cognitive Radio proposal to Air Force Research Lab

Project: Design and Development of Cognitive Jamming (Funded by Air Force Research $\mathrm{Lab})$ 